Article

\title{
The Antithesis of Entropy: Biosemiotic Communication from Genetics to Human Language with Special Emphasis on the Immune Systems
}

\author{
John W. Oller, Jr.
}

Department of Communicative Disorders, PO Box 43170, University of Louisiana, Lafayette, Louisiana 70504-3170, USA; E-Mail: joller@louisiana.edu

Received: 12 January 2010; in revised form: 9 March 2010 / Accepted: 24 March 2010 /

Published: 31 March 2010

\begin{abstract}
Entropy can be defined as the antithesis of well-formed true reports that agree with each other and with the material facts accessible through the experience of one or more competent observers. The abstract convergence (strictly formal, logical agreement) of true narrative representations (TNRs) — ordinary valid reports of facts of experience - makes them formally more complete than fictions, errors, lies, and nonsense. A limit of absolute entropy is theoretically reached if all resemblance to a TNR is lost. As argued here, TNRs - formally defined along the lines of Peirce's exact logic — provide the necessary foundation for functional human languages and for biosemiotic systems. The theoretical concepts of pragmatic mapping-the fitting of a TNR to whatever facts it represents - and the constructive cycle of abstraction that enables a child to discover the systems underlying such mappings are introduced and illustrated from child development and then shown to apply to the human neuroarchitecture, genetics, fetal development, and our immune systems. It is also argued that biological disorders and disease conditions logically must involve corrupted (damaged, undeveloped, or otherwise incomplete) representations at one or many levels.
\end{abstract}

Keywords: abstractive cycle; biosemiotics; brain architecture; embryological development; immune systems; information theory; language acquisition; learning to read; human neuroarchitecture; pragmatic mapping; self-consciousness; sign hierarchy; systems grammar; theory of abstraction; true narrative representations 


\section{Building a General Theory of Signs}

Since this treatise is not a stand-alone book but is rather a longer-than-expected contribution to this special issue of Entropy, as pointed out by several anonymous expert readers and the distinguished invited editor of this issue, I have been asked to put a paragraph up front about its content and length.

Four major sections are incorporated: Section 1 deals with both the development and testing of a general theory of sign systems (general semiotics) by examining the early cognitive and linguistic development from birth forward. That section, I believe, is essential reading, providing the necessary groundwork for the three sections that follow where the theory is applied respectively to the neuroarchitecture of the human brain and body in section 2; to genetics, epigenetics, and certain aspects of biochemistry in section 3; and to the immune systems incorporating critical elements of embryological development in section 4. Because of the various threads running throughout the whole, it was decided not to set any one or more of the parts aside as appendices, but rather to advise readers here at the start concerning the organization, and to invite them, if they wish, to pick and choose their sections. The coherence and intelligibility of the general theory depends mainly on sections $1.1-1.5$ (roughly the following 8 pages). To appreciate the depth and power of the general theory will require digesting also the parts about the abstractive cycle and the multi-layered sign hierarchy (sections 1.7-1.9; an additional 8 pages), but specialists in any of the areas dealt with in subsequent setions may prefer to skip over the latter half of section 1 to go directly to section 2, 3, or 4 .

At any rate, with section 1 as background, it should be possible to treat any one or pair of the subsequent sections more or less as stand-alone applications of the theory. The one thing that comes clear in thinking through the applications of a general theory of signs to biosemiotic issues is that this chapter is more susceptible of expansion than of abbreviation. With all the foregoing in mind, I want to thank the editors of Entropy, particularly the anonymous readers and Søren Brier who helped me to improve this particular contribution (any remaining errors being my own). Knowing in advance, as C. S. Peirce wrote in the preamble to one of his many "screeds" as he called them, that you are "one among millions," I also thank you the reader in advance for devoting your brain cells, along with the caffeine, patience, and persistence requisite to the task just ahead.

\subsection{Truth and Meaning}

The idea that truth and meaning are necessarily related can be dated at least as early as $401 \mathrm{AD}$ when Augustine argued that infants learn their native language by associating the words of other persons with things that those other persons often point out to the infant [1]. Augustine's description of the processes underlying association adumbrated a much richer conception of the basis for meaningful sign systems that would be developed in the latter quarter of the 19th century by C. S. Peirce who embraced and expanded on the "correspondence theory of truth" [2]. Peirce also generalized the Boolean algebra of binary relations to provide a systematic and complete logic of all possible relations - a theory he called "the logic of relatives" [3]. In 1879, Frege suggested - but did not try to prove because he evidently took it for granted - that all meaningful signs depend on the sort of referring relation seen between a name and its object [4]. Later, Tarski [5,6] would prove mathematically that the sort of relation found between a referring term correctly applied to its object and the object referred to is precisely the kind from which all meaning must be constructed. 
Following that underlying theme, in this paper, I want to discuss a general theory of sign systems grounded in Peircean ideas [7,8]. Elsewhere, together with several colleagues, I have demonstrated applications of the general theory in question to discourse processing [9] and to the foundational concept of validity in scientific measurements [10]. Here, the theory of true narrative representations (TNRs) along with the process of pragmatic mapping that underlies that theory and the constructive cycle of abstraction, are all introduced and illustrated in the context of child language development. Then, they are shown to be relevant as applied (somewhat independently) to human neurological and somatic architecture, to genetics, epigenetics, and biochemistry, and to our immune systems from gestational development to maturity. There is no doubt that the whole discussion could be more elegantly and succinctly organized, but my purpose here is to make the gist of the applications comprehensible, so they can be opened up to critical examination by many pairs of eyes. The point is to find a traversible path now, with the hope that the discovery of an even better route will soon be discovered later on.

\subsection{The Deeper Quandary}

It is intriguing to wonder how it came about that we are able to consider the possibility of a general theory of signs. To many, including C. S. Peirce, the ultimate question is how we got here. Addressing that question was a challenge that Peirce himself could not pass up. In the final analysis, it seems that all of us must undertake the difficult task of understanding how physical material itself could come into existence, perhaps from nothing; how it could become differentiated into a considerable diversity of complex chemical elements and the vast distribution of matter and energy in space and time; and ultimately how all that material stuff could somehow come to be arranged as it now appears in the diversity of living organisms including ourselves - with our capacity to wonder about ourselves and our situation. We ask, what and why, and who am I? How did I get into this wonderful predicament. Peirce seems to have referred to this quandary obliquely as "the riddle of the Sphinx" [11]. It is a difficult one inasmuch as it either seems to demand that we should know, as Einstein is supposed to have suggested [12], the mind of God, or, alternatively, to show how the cosmos could have engaged in what Luhmann calls autopoeisis [13]. Brier [14] expresses the nature of our quandary insofar as "we still do not know for certain and in specific detail what 'the outside world' is; and even less so [do] we know what or who 'we' are" (p. 149). Yet, the problem presents itself to us in our mysterious, ineffable, and intangible self-consciousness - as Brier so eloquently illustrates throughout his works, but especially in his masterful 2008 book [14], Cybersemiotics, which consolidates, extends, replaces, and supercedes his publications up through 2006 (see pp. 15-18 in that work).

It would seem that the highest level of representation that demands explanation is the sort that we find in the human language capacity that enables us to concern ourselves with our own material, mental, and social existence. The enigma we face concerns not only the body, the mind, and the whole neighborhood, but all of their interactions. Much energy has been devoted to generate order from chaos by deterministic mechanisms, some of them enriched by information theory, but all of which, as Brier [14-16] has pointed out, fail to achieve anything like a satisfying explanation of human selfconsciousness. It is his hope, as I understand him, to establish what he calls [14] a "third culture" bringing together the seemingly disparate approaches of "science-technology, and the humanities and 
social sciences" (p. 13). He rejects the traditional view of "information" conceived as a disembodied abstraction along the lines proposed by Claude E. Shannon in 1948 [17]. A crucial difficulty in Shannon's approach was contained in his definition of information so as to exclude the very essence of ordinary reference, meaning, and all the processes by which references and their meanings are developed and understood. Shannon wrote: "Frequently the messages have meaning; that is they refer to or are correlated according to some system with certain physical or conceptual entities. These semantic aspects of communication [among which he also inadvertently discards here all pragmatic aspects of reference to objects, bodies, minds, and the events of the world of experience] are irrelevant to the engineering problem. The significant aspect is that the actual message is one selected from a set of possible messages" (p. 379).

It is no small problem that the mathematical theory of information regards every expressible thought or emotion about absolutely anything as if it existed in some perfectly determined and pre-existing list from which communicators could instantaneously select any desired "message" ad libitum. Yet, in ordinary experience this is surely not the case. The possible messages of ordinary discourse are uncountably many-effectively an infinitude of uncountably many multitudes - that absolutely cannot be determined in advance by any stretch of the imagination. Nor could they be stored, much less successfully searched-even if we were able to call upon all the current cybersystems of all the computers of all the present world. Brier [16] also examines traditional definitions of information stemming from Shannon's in light of various other theories and reaches the inescapable conclusion that information "is not a sufficient concept for understanding cognition and communication" (p. 429). Furthermore, Brier explicitly notes (p. 22ff.) that the idea of abstracting information still further by defining it as "negative entropy" or the opposite of its complete absence — as argued especially by Norbert Wiener [18] — cannot provide the required sort of referential meaning of living, at risk, bodily persons taking note of actual facts in a nondeterministic common world of experience where time and chance demand attention.

All efforts to explain how deterministic systems might mechanistically improve themselves indefinitely to the point of even a single celled organism, not to mention a fish or mammal, or a talking ape, have so far come up short of a viable specimen. The trouble is not merely to get a genetic script in order, but to find an actor that can read it. Wills [19] wrote that “. . . genomic sequence information can be interpreted as biological instructions and executed as a genetic code script only by a suitably prepared cell with which the program is in proper registration" (p. 345).

\subsection{Defining a More Tractable Problem}

Setting to one side the seemingly intractable problem of finding a deterministic way for matter to lift itself by its bootstraps to the top of "Mount Improbable" [20], perhaps a more promising way to search for a path from minimal conscious representational capacity to our present state of knowledge, might be to follow the road marked out in the development of any normal child in acquiring a language. In any case it is easier to do that, presumably, than to begin at the top of the mountain with the adult understanding of abstract metaphors, irony, and the like along the lines of Lakoff and Johnson [21] - as worthy a goal as that may be. Or, more challenging still, is the sort of valiant effort undertaken by Subrata Dasgupta [22] to develop a computational theory of scientific creativity as 
applied in the invention of microprogramming which many, including Dasgupta himself, attribute to the British computer scientist Maurice Wilkes.

Wilkes's concept of microprogramming, as understood by Dasgupta (p. 92), led to the metaphor of computers within computers calling to mind the various homunculus theories of the human brain. Interestingly, Dasgupta sought not only to apply the computational metaphor as an explanatory theory of the creative invention of microprogramming by Wilkes - a theory that Dasgupta argues is at a minimum both plausible and testable against the available historical documentation and the writings of Wilkes himself. Dasgupta proposes a series of logical steps (inferences) as a possible route leading eventually to the technological advances that have greatly impacted modern information processing, storage, and retrieval systems largely through the creative work of Wilkes. Except for the contribution of Dasgupta himself as the producer and developer of the proposed "computational" theory of Wilkes's scientific creativity, we might almost imagine the homunculus - a tiny but potent and surely a brave little image of Wilkes himself - climbing out of the microprocessor deep within the bowels of the computer to describe the logical processes engaged in by his maker. But the marvelously creative contribution of Dasgupta himself in producing the allegedly "computational" theory of the scientific creativity of Wilkes remains a nontrivial and intrinsically unexplained component of the ongoing discourse. Is Dasgupta too modest in backgrounding his own part in the invention of his theory about Wilkes's creativity, or is he fully aware that his description of the "computational theory of scientific creativity" end up by presupposing what it is supposed to explain?

It is the capacity to produce and engage in meaningful discourse that demands attention as Brier [16] has argued (see especially pp. 378-380) and I would add only that explanations that do not account for the surpassing uniqueness of ordinary discourse must also fall short in trying to account for creativity in the higher sense targeted by Dasgupta. Simply put, mechanistic explanations, however useful they may be as metaphors, are certain to come up short as explanatory models of the semiotic systems employed by real persons - and, by the way, I do not think Dasgupta would disagree here.

By contrast with evolutionary approaches seeking to supplement "mechanisms" and "deterministic" thinking, in looking to the major milestones of early cognition and language in infancy, I believe it is possible to make some genuine progress by showing how and why the fundamental operation of referring to something by name provides the necessary basis for all meaningful sign systems. Incidentally, Brier [14] (see p. 262 specifically) as well as Dasgupta [22] (on pp. 33-34), along with the other brilliant thinkers and theoreticians that each of them cite, take note of the fact that the association of ideas from different representational systems are critical to the comprehension of metaphors and abstract sign systems in general. There is, I believe, a germ of insight in all these efforts to understand how such associations are made. In Brier's discourse on Cybersemiotics, we find references to couplings, connections, coordination of coordinations, and so forth; and in Dasgupta, emphasis is placed on the "bisociation" (a term from Arthur Koestler [23]) critical to the use of metaphors in scientific discovery and in referential reasoning. Both Brier and Dasgupta appeal, as I do, to the logical processes of abduction, induction, and deduction as discussed and developed most extensively by C. S. Peirce [25]. However, as I will show here, the grounding of all such rational processes and of all meaningful sign systems depends on the sort of true narrative representations (TNRs) and the processes of abstraction by which infants are initiated into ordinary human discourse. It is that initiation that forms the basis for the discovery of the conventional linguistic signs that are 
associated with actual facts in the common world of experience. If we understand the steps of that initiation process, the basis for discovering the meaning of conventional sign systems of surpassing complexity, I believe we can make some progress toward a thoroughly grounded and general theory of signs.

It is often noted that Gregory Bateson spent years analyzing the social nuances of a few minutes of filmed adult dinner conversation [24]. With Bateson's revealing work in mind, it is all the more remarkable that, within about a year after its birth, a normal human baby works out the first 10 layers of the 30 layer hierarchy of sign systems evidently undergirding all human languages [8,26]. It takes 12 to 20 years, however, to attain the most abstract level of that hierarchy. So, it makes sense to pay close attention to the logically simpler early steps in building up the first meaningful signs attained by human babies. As theoreticians, we can look to the earliest stages of the processes of language acquisition by infants with the reasonable hope of being able to understand them on account of the fact that, in practice, all normal human children make child's play of those initial stages of semiotic development and language acquisition.

Our theoretical excursion into the earliest semiotic systems of human infancy will also provide a rational basis for the further applications of the theory to be introduced in this paper. After summarizing major findings of its application to early cognitive and linguistic development, it will be possible to introduce some more recent applications to the neuroarchitecture of the adult human brain and then to the whole human body as it develops from conception forward. In such a theoretically developed context, it will also be possible to show how the fundamental forms and functions of TNRs and their essential components are ubiquitously manifested in the genetic systems that form the basis for the creative unfolding of bodily systems, and in the essential functioning of the deeply layered human immune systems. Our entire excursion will, I believe, show that all these interdependent communication systems must be and are grounded at their basis, in their architecture and functioning, on valid (factual) representations of the sort found in coherent true reports of the narrative kind-TNRs.

The exploration of the creative and constructive development of sign systems from early infancy up to at least the first meaningful word was an appealing path for adult researchers because we could hold out the reasonable hope that as adults perhaps we ought to be able to follow the path marked out by infants. For the same reason, to the extent that the initial expectation proved to be correct, what was learned from early child development and especially from early language acquisition, seemed a good place from which to launch an introduction and further exploration of the emerging general theory of signs. Giving credence to such a plan was the fact that a series of logicomathematical proofs have been developed showing that all meaningful sign systems accessible to any human infant utterly depend on the relatively simple sort of valid reference to someone or something by its conventional name (as suggested by Augustine, Peirce, Frege, Tarski, and others-recall the references to coupling, bisociation, etc.). Moreover, empirically testable inferences drawn from those theoretical proofs have been examined in a considerable variety of contexts concentrating, as Peirce and others have recommended, on the most surprising (unlikely) hypotheses first [7-10,25]. For example, it was surprising to infer, as we did from the theory of abstraction (to be explained herein) that any normal baby should theoretically be able to learn to read printed words, symbols, and phrases well before the same infant is able to say the words or name any of the letters or strokes used in producing their 
printed forms. The general sign theory to be discussed here predicts this outcome and it has been shown to be correct in the research and video documentation of actual learners by Robert J. Titzer. Many babies, with the help of adults using methods made clear by Titzer, by about six to nine months of age [26,27] have been able to learn to read - in fact it is theoretically possible for unimpaired babies to learn to read printed words and phrases with excellent comprehension before they can say the words out loud. This surprising outcome is predicted and explained by the theory of abstraction which flows seamlessly from the theory of TNRs along with a theory of systems grammar discussed elsewhere $[28,29]$.

\subsection{Why Ordinary TNRs are Foundational}

Multiple logicomathematical proofs converge on the inference that true representations of the most ordinary sort, e.g., reports of events that are consistent with the facts in, say, a simple naming relation (as in Figure 1, parts A and B), must form the foundation for all other meaningful sign systems without any exceptions. For instance, it has been proved that the construction of any meaningful fictions, the discovery of errors (where they can be discovered), and the appeal of any lies whatsoever (in those cases where the lies are sufficiently well conformed to the requirements of true representations to seem plausible) depends at its foundation on previously developed and instantiated TNRs. Peirce [30] seems to have had these in mind when he wrote, “. . . the most perfect of signs are those in which the iconic, indicative, and symbolic characters are blended as equally as possible” (§4.448, p. 361).

Figure 1. (A) The fundamental Peircean trichotomy and the three necessary parts of a true narrative representation; (B) the pragmatic mapping triad; and $(\mathrm{C})$ the abstractive cycle.
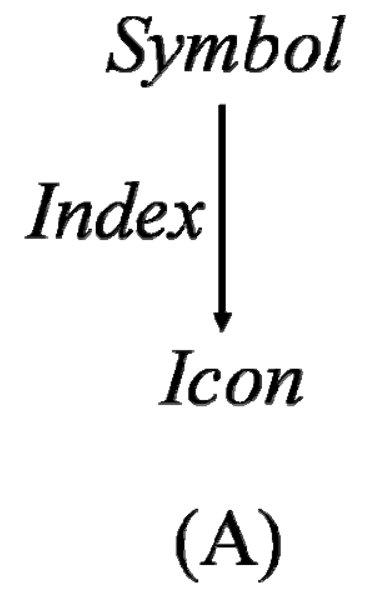

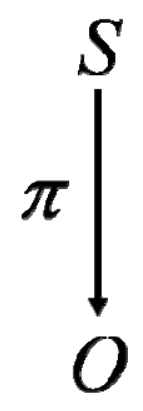

(B)

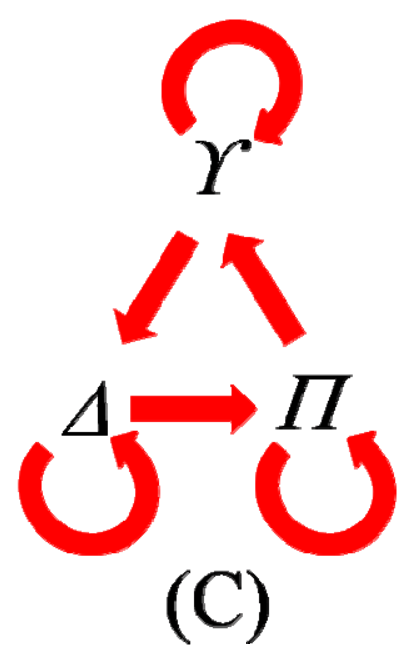

(C)

The proof can be summed up as follows. All sign systems must fall within the limits defined by simple true reports, at one extreme, and complete nonsense, at the opposite. In actuality, there can be no form of nonsense that reaches the theoretical limit of complete entropy, but it is possible to define that limit by appeal to its antithesis - the sort of truth found in ordinary true reports of actual events lived through and known to one or more competent observers. It takes a bit of logical work to prove that the upper limit on meaningfulness can only be achieved by the relatively perfect sort of representations found in TNRs. This is done by showing that all fictions, errors, lies, and any form of 
nonsense must fail to achieve the relative completeness (perfection in the logical and grammatical sense) of TNRs. It can also be shown that because all possible representations must fall between the upper limit of relative completeness found in ordinary TNRs and the lower bound of complete nonsense (the limit of entropy), it follows that only TNRs have the logical properties necessary to enable (1) determination of meaning, (2) valid indexical (deictic) connections with the matter/space/time continuum of ordinary experience, and (3) generalization of any fully abstract (and thus arbitrary) symbol across distinct contexts of experience or imagination. Thus, on a strictly formal basis, it can be proved that all fictions, errors, lies, and gradations of nonsense short of the absolute (and purely theoretical) limit of complete entropy depend on the abstraction and generalization of meanings that can, ultimately, only be discovered through instantiations of ordinary true reports-TNRs. All of this follows by virtue of the fact that without any such instantiation, any abstraction is demonstrably void of any discoverable content whatsoever.

The foundational claims for ordinary TNRs are strong, completely general, and surprising to many theoreticians - especially to those who may have embraced the long tradition of regarding the naming relation as a naive basis for any theory of language or cognition. Actually, this should probably not surprise anyone on account of the fact that traditions that have despised the days of small beginnings have often turned out themselves to be naive and simplistic. As Peirce scholars have often observed, simple ideas are generally to be preferred. Nevertheless, the implicit admonition that the surprising inferences of a general theory of signs must be critically examined both from a logicomathematical perspective (as pure abstractions) and from whatever empirical points of view can be brought to bear must also be taken to heart.

To whatever extent the goal of developing a general theory of signs may have been achieved, it follows that its consequences should generalize across sign systems ranging from the molecular level of systems found in genetics through the higher epigenetic bodily systems of neonatal development, along with the internal systems of digestion, metabolism, repair of injured cells or tissues, defense against disease agents, and so forth, right on upward through the macro-levels of bodily and neural architecture, to psychological and social systems at the highest levels. In this paper, I concentrate first on the psychological and social sign systems involved in language development - the highest type of sign systems and the ones that enable us to have this discussion in the first place-and then I go on to show applications of the theory to the human neuroarchitecture and the human body itself. The body, it can be argued, stands at or near the center of the representational systems of interest to human beings. Based on inferences to be drawn directly from the simplest sort of TNR - the kind found in a validly applied predication or name - to show first that a general theory of signs to human language, the most abstract and highest representational system to which we have access, and then to work from the commanding molecular level of genetics, through neonatal development, and to the remarkable communication systems of the body's internal defenses.

\subsection{Pragmatic Mapping}

At the very basis of meaningful sign systems we find the sort of pragmatic mapping relation - the simplest and most general form of a TNR — that can be formally summed up as shown in the naming relation diagramed in Figure 1. It can be more simply written in the formula $S \pi O$ where $S$ represents an 
abstract symbol (usually expressed in one or more words), $\pi$ represents a signifying or referential relation connecting the $S$ in question with its logical object, and, $O$ represents the particular logical object in question that is determined by the $S$. It can be shown that in the simplest such mapping relations, e.g., say, a naming relation, where, for instance, the name "George" is applied to the person GEORGE known by that name, the predication is as true as it purports to be of its logical object in its actual context. Also, it comes out that since the person GEORGE can only exist in an actual relation to the conventional predicate "George" in some real context of experience, any ordinary true representation taking such a form must be of the narrative kind where bounded objects interact over time through events that unfold as a spatio-temporally constrained sequence that might be represented in a true report or narrative about George. It can also be shown logically and empirically that the active agents (the protagonists) along with the time-space coordinates of events in any true report give the narrative its coherence. Such referential connections constitute its meaningful content and provide the grounding for valid inferences of all types-abductive, inductive, deductive, and combinations of them.

Also, as long as we allow the terms of the formula to represent either simple or complex sequences of elements, the formula can be generalized to all TNRs. Given that fact we may similarly capture the form of all fictional representations in the formula, $S \pi \underline{\underline{O}}$, where the added underline suggests the fact that in a fictional representation (e.g., in fantasy, imagination, or in the expression of a scientific hunch or untested hypothesis), all or at least some part of the logical object, $O$, of the fictional representation must be imagined. For instance, suppose we refer to a non-existent person which we arbitrarily designate by whatever name we like. In such a case, the person named must be imagined. Hence, the underlining of $\underline{O}$ to suggest that all or some part of it must be supplied by imagination as in the filling in of a blank, or guessing the value of some unknown. Similarly, we may generalize the representation of any error in the formula, $\underline{\underline{S}} \underline{\underline{\theta}}$, where the additional strikethrough shows that in an error all or some part of the symbolic element in the formula is mistaken (would have to be replaced to make the representation valid) and, therefore, the $O$ at hand is not the one signified and is for that reason also marked with a strikethrough. A simple naming error may be construed as a prototypical instance. For example, if the name "George" is intended to refer to the person GEORGE, but the person "RANDOLPH" is actually at hand, both the symbol, $S$, and the object, $O$, are fictionalized (therefore underlined). Because in an error the symbol, $S$, does not correspond as intended to the object, $O$, named, in the generalized error formula both are marked with a strikethrough. Extending the same logic, we may construct a general formula for lies as $\underline{S \pi \theta}$. In all lies, it can be shown that all three of the elements of an ordinary true representation are corrupted in the manner of fictionalizing them; hence, the underlining. Also, all three elements are presented in such a manner as to generate an erroneous representation of the facts in question; therefore the strikethrough marking of each element. Additionally, the redlining is used to call attention to the fact that formally every lie is intended by its creator in such a manner as to deceive an interlocutor into regarding the lie (a known falsehood) as if it were true. For instance, if O. J. Simpson murdered his wife, his claim that he was in Chicago when her death occurred in Los Angeles is false with respect to the symbols, the indexes, and the icons involved in the representation as shown in the formula for a lie.

If we look for a form more corrupted than a lie, we can only come to the sort of representation that would have to be classified as some form of nonsense or mere noise. At the limit of complete 
randomness we come to a limit of entropy which, it may be argued, is the antithesis of the foundational logical form, $S \pi O$. It remains to show that the foundational, simplest form of a referring relation is the sole source for the necessary building blocks for all higher and more complex representational forms. This can be proved to a fare-thee-well in the logicomathematical way, but it can also be illustrated less technically in a manner that is irrefutable. Try to imagine how you might supply the missing $O$ in a fictional $S \pi \underline{O}$ if you did not know beforehand the meaning of the $S$. Logic shows that the fictional referent of a sign whose possible meanings range from everything to nothing is completely indeterminate. That is, its meaning cannot be discovered without associating the $S$ in question with some $O$. The problematic barrier to the discovery of the meaning of fictional representations that are not preceded by simple referential instantiations of the $S \pi O$ form is not only insurmountable in the case of fictions, but also in the cases of errors and lies. As a result, it is easily seen that the $S \pi O$ form is foundational to the discovery of any meaningful fictions, errors, or lies. But, since all representational forms that have any determinate meaning (i.e., that are not uninterpretable nonsense) must take one of the logical forms described, it follows that the $S \pi O$ form is foundational to all possible signs. Q. E. D.

Summing up the series of arguments starting from the formal nature of a lie, if the producer did not intend to deceive, the representation would be reduced to an error. If the error turned out not to be taken as true by its producer/interpreter, it would be reduced to a mere fiction. If the fiction, in turn, turned out not to be imaginary, but a valid representation of an actual fact, then, the representation would be reduced to the simplest logical form which would turn out to be a TNR of the $S \pi O$ sort. Logically, it can also be demonstrated that simple true representations are formally less complex than fictions, which in turn are less complex than errors, which in their turn are less complex than lies. Moreover, relative to all others, the $S \pi O$ type of representation is absolutely unique in three important logical properties: (1) The $S$ of any such relation is relatively well-determined in the material sense through its valid association with its $O$ which consists of particular content in the real world of space, time, and matter. (2) It follows from the unique material determinacy of the content of such an $S \pi O$ relation that it uniquely also provides a valid source of uncountably many necessary logical inferences - including valid probabilistic judgments - about transitive relations connecting its $O$ with the rest of the space, time, and matter world. (3) From both of the former logical properties, both formally unique to the $S \pi O$ relation, it also follows that the $S$ of any such $S \pi O$ relation provides a valid basis for the abstraction and generalization of its conventional uses (and therefore its material content). Indeed, on a strictly formal basis it can be shown that only true representations of the $S \pi O$ shape can provide a sufficiently determined and connected basis for such abstraction and generalization.

\subsection{Testable Inferences}

Among the testable inferences (hypotheses) flowing from the general theory of signs and its formal elaborations are the following: To the extent that all other factors can be held equal, true representations must be (i) easier to comprehend, (ii) acquire, (iii) recall, and (iv) produce than fictions, errors, lies, or increasingly corrupt forms of nonsense. Another inference that flows from the theory is a simple and straightforward logicomathematical proof showing that in the special instance of a sequence of reportable events, A, B, C, and so forth (ones that occur in the order 1, 2, 3, and so on), a chronological narrative report mapping $\mathrm{S}_{1}$ to $\mathrm{A}, \mathrm{S}_{2}$ to $\mathrm{B}$, and so on, in the reporting of the sequence is 
the simplest possible mapping [9,10]. Moreover, chronological narrative reports, in general, including fictions, errors, and lies (all else being held equal) will inherit all of the empirical consequences iterated in Roman numerals (i-iv). The expected benefits of chronological fidelity are easily testable with respect to fictions and, indeed, are empirically valid in crucial tests. For instance, Ohtsuka and Brewer [31] showed that fictional narratives told out of order were universally harder to process than the same fictions presented in a chronological order and that fictional narratives with a flash-forward (from an early event to one considerably later in the sequence) proved almost impossible to comprehend. So far, competing alternatives (including various null hypotheses) have had to be rejected while even some seemingly unlikely necessary inferences derived from the general theory of signs have been convincingly confirmed in studies where a different empirical result would have required rejection of the general theory [9].

\subsection{The Abstractive Cycle}

Next, consider the fact that the $S \pi O$ relation expresses a logical hierarchy of elements which may be termed, icons (as shown in the $O$ ), indexes (as expressed in the $\pi$ ), and symbols (as signified by the $S$ ), all of which is summarized in the two left-most triads of Figure 1 (A and B). It is interesting that this mapping relation also implicitly contains, therefore, what has been termed the abstractive cycle $[8,26]$, as summed up in the right-most triad of Figure 1. It must be noted that in developing a constructive theory of language acquisition there is little need to bother with fictions, errors, lies, or nonsense on account of the fact that the only sufficiently complete pragmatic mappings on which to initiate the discovery of the conventional meanings of abstract linguistic signs are ones that are sufficiently wellformed to be termed "true" in the most mundane and ordinary sense of "truth."

It may be useful to note that Danesi's proposed laws of second order cybernetics [32], following Brier [16] and others (see Danesi's references in this volume of Entropy), applied to the problem of explaining language change, also seem appropriate here. TNRs, in general, provide optimal examples of the principle of least effort by which a dynamically well-fitted representation is to be preferred over one that does not fit so well. Moreover, there is a great deal of evidence in the child development and language acquisition literature encouraging us to believe that it is surprise-value caused by discovering imperfectly fitted representations - ones that generate errors detectable by the advancing learner - that drive the advances upward through the various phases and tiers of the cognitive-linguistic sign hierarchy [26]. Such an observation is consistent with Peirce's suggestion [33] that a child's self-awareness begins with the discovery that the facts of experience as seen by others may differ from his or her own views. For instance, the "child hears it said that the stove is hot. But it is not, he says" (p. 202); also cited in [14] (p. 404) and in [26] (p. 22). Subsequently, an empirical check by the child turns up the error. Likewise, Jean Piaget [34], the Swiss biologist turned psychologist, observed that "the child first seeks to avoid contradicting himself when he is in the presence of others" (p. 163). At any rate, advances up through the various layers of sign systems in the vast hierarchy to be constructed by the child are generally preceded by the discovery of false expectations - ones that force a re-analysis and reformation of representations to get them to conform more closely to observed facts. The movements within the distinct phases of the cycle of abstraction generating that sign hierarchy 
(see the rightmost diagram, C, in Figure 1) at its basis can be inferred from the simplest of complete pragmatic mappings.

The abstractive cycle consists of three phases: first, there is a discriminating or iconic phase, $\Delta$; second, there is a prescinding (moving) or indexical phase, $\Pi$; and, third, there is a hypostatizing or symbolic phase, $Y$. Interestingly, these three phases must occur in the order just named. The discriminating phase involves the discovery of boundaries associated with a particular object, call it $O$, which enables the production of any number of icons of that $O$. The prescinding phase involves movements of either the observer (also representable in a bounded icon) or of the $O$ observed. This second phase enables the production of any number of indexical signs connecting the observer to the various locations of the object observed as one or both of them move about in space and time. The hypostatizing phase of abstraction enables the production of a fully abstract concept of an $O$ as distinct from its percepts and the observed or imagined inertial trajectories or locations of those percepts.

The prescinding of an icon cannot occur without the prior discovery of the boundaries of the icon itself. The logicomathematical proof of this proposition is also borne out by empirical demonstrations of the fact that it is impossible for an observer to detect the existence of any object in any field of perception without discovering the boundaries (edges and/or surfaces) that set the particular object in question apart from its context [35,36]-hence, discriminating absolutely must (in the logical sense) and does (in the empirical sense) precede prescinding.

In its turn, without the discovery of the continuous connections between the distinct locations of a moving object by prescinding its icon which maintains its boundaries as the object moves, i.e., its trajectories of motion defined by the continuity of the moving boundaries of its icon, it would be quite impossible to discover the fact that a moving object/person retains its identity across distinct locations. Without the latter discovery having been established as a fait accompli by the prior prescinding of a discriminated icon, the hypostatic conception (actually, the imagining) of an object that is no longer within the field of perception when it is to be imagined would be logically impossible to construct. Hence, prescinding must precede hypostasis.

Again, this can be proved by exact logic along the following lines: If there were no constraint on the concept to be produced (the object to be conjured from imagination) from the discriminated icons of that particular object (i.e., if there were no constraints emanating from its percepts), and if there were no discoverable connection whatsoever between the distinct inertial locations of the actual particular object to be conjured in imagination - that is, supposing that all the actual trajectories of motion of any object (or the trajectories of a possible observer relative to that actual object) were totally unknown, how would it be possible for such a person to associate any imagined icon whatsoever with that particular object? To do so would be akin to producing a valid sketch of an object whose shape, size, surfaces, materials, density, etc., were completely unknown to the person drawing the sketch.

Thus, the order of the progression from discriminating $(\Delta)$, to prescinding $(\Pi)$, to hypostatizing $(Y)$ is a matter of logical necessity as well as of empirical fact. From a developmental linguistic/cognitive perspective, infants must construct perceptual icons ahead of the tracking of their movements and both of these accomplishments must precede the discovery of associations between arbitrary significant movements (gestures or words) and the objects which they signify. The discriminating of one or more icons is also required to initiate the sign hierarchy (the 30 levels summed up in Figure 2). Failing any 
discrimination of any bounded objects in experience, the individual would-be learner will remain unable to represent anything and thus unable to learn anything. The theory shows that the first phase of the first abstractive cycle produces one or many discriminated icons (percepts of objects); then, the second phase produces one or many prescinded icons (e.g., the recalled percept of an object that moves from one location to another within the perceptual field); and, finally, the hypostatic phase produces one or more icons that are fully abstracted from their context (i.e., concepts of objects disregarding, or fully backgrounding, any particular perceptual field in which the conceptualized object might appear).

Figure 2. The abstractive cycle and multi-tiered and multi-layered hypothesized sign hierarchy.

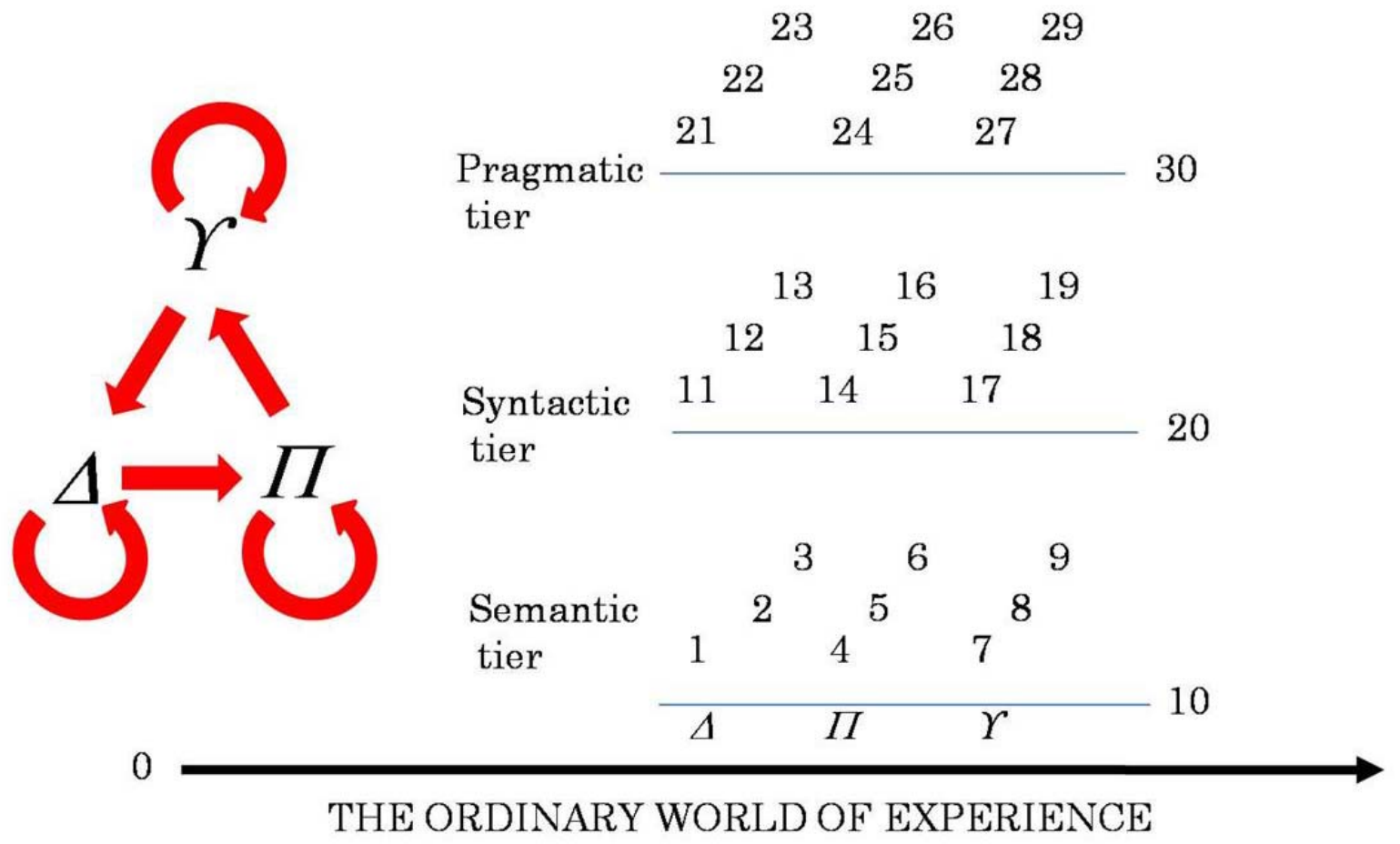

For the foregoing reasons, it follows by exact logic requirements that the iconic cycle of abstraction (depicted in Figure 2 as the $\Delta$-cycle of the semantic tier of the hierarchy to be built up by the developing sign user) must occur before the $\Pi$-cycle. This follows from the fact that it is impossible to conceive of any particular line of motion of, to, or from a particular object until the object itself can at least be represented as a hypostatic icon. To illustrate, how would we think of any object not present in the perceptual field if we had no valid abstract concept of it? Yet, without a concept of an object not present, how could that object be thought of, much less searched for in any given location? To think of something not present, or to go hunting for it in the perceptual field, a hypostatic icon is a minimal prior requirement. That is to say, one or more sign products of phase 3 of the iconic cycle of abstraction must be available to represent the stream of experience (the 0th level of Figure 2) prior to the construction of any signs at level 4 of the developing hierarchy - that is, at the first phase of the indexical or $\Pi$-cycle of abstraction. In fact, it is the case that the products of the just prior level of 
abstraction are essential building blocks in every case for the signs of the next level upward throughout the hypothetical sign hierarchy.

Returning to the first phase, then, of the indexical cycle-Picking up the path of the unfolding successive levels of sign systems at the completion of the iconic cycle-supposing that the entire iconic cycle of abstraction has been completed, it is just possible for the developing sign user to begin to notice significant movements of objects with the assistance of the existing repertoire of already established iconic signs - discriminated, prescinded, and hypostatic icons, that is. This is not to say that the indexes are not instantiated in experience beforehand, but they cannot be noticed as distinct entities until and unless all three layers of icons are already in place. Here is why: in order for an infant to prescind the icon of a particular object (i.e., to imagine a percept in a location from which the object itself has been displaced), it is first necessary to discriminate the icon to be prescinded and projected by imagination into that location. Similarly, if a completely abstract icon is to be constructed (thought of) when the object that has previously occasioned the production of both discriminated and prescinded icons is not present in the perceptual field of the infant, the prior steps of discriminating and prescinding icons of that object must have already taken place.

Following the same line a step further, it is clear that the infant cannot think of an object in a location where the object is not present without representing the object itself as a fully abstract concept, a hypostatic icon. However, once the hypostatic icon of that object has been achieved, it will become possible to notice that the object formerly located at a particular place in the perceptual field is no longer there. It is worth noting that the semantic basis of negation is implicit in the surprise of the infant who discovers that the object formerly located at a particular place in the perceptual field is not there now. With that observation in mind, it can occur to the infant to look for the missing object. What was amazing to researchers studying infant psychology - for instance, see the experiments by T.G.R. Bower [37] summed up as early as 1971-Though it is plainly predicted and is explained by the theory of abstraction [26], is that an infant cannot logically know that an object which moves about from one location to another is the same object until and unless the movements of the object can themselves be represented. Yet, to do so will require the formation of indexes that cannot be formed until after the iconic cycle of abstraction has been completed-perhaps many times over with distinct icons.

In order for the infant to conduct a successful search for a particular object, the infant cannot merely look in all possible places with nothing in mind. The result of a search of that kind would no doubt find many objects, but could not be said to be a search for any object in particular. To conduct a search for a particular object, like a driver looking for a particular set of car keys, the infant must have in mind a concept of the missing object in order for a search for that particular object to succeed. When such a search occurs with success, the infant will have achieved what may be termed the discriminated index (level 4 of the sign hierarchy described in Figure 2). At this point the infant has achieved the first phase of the indexical cycle of abstraction. The second phase will require that the infant coordinate a series of its own movements with the movements of an object or person that changes positions within the infant's field of perception. In doing so, the infant will achieve the level of prescinded indexes (level 5 of Figure 2). Once that is accomplished, it is possible for the infant to notice the disappearance of an object that it can track in the perceptual field with a prescinded index when the tracked object disappears beyond the horizon of the perceptual field. At that point a 
hypostatic index (level 6) is required to keep in mind the object that is still out there beyond the perceptual field. When that occurs the indexical cycle is complete and the first phase of the symbolic cycle can come into view.

Until it is possible to think of an object that has passed beyond the horizon of the perceptual field, it will hardly be possible to compare it with any other that enters the perceptual field, or to judge the distinct appearances on different occasions to be the same or different. Without that capacity of judgment, it will be impossible to discern the repetition of a word, for instance, or to discriminate any one symbol, say a printed form, from any other. Yet, such a discrimination (level 7, and the first phase of the symbolic, $Y$-cycle of abstraction) must logically precede the deliberate reproduction of even the surface form of any word or symbol as in repeating a syllabic sequence (e.g., as in infant babbling; level 8 in the hierarchy of Figure 2), or copying a movement (as in imitating the pointing gesture).

The third phase of the symbolic cycle is initiated, usually several weeks later, when the child discovers that the distinct surface forms of speech (sign or print) have distinct meanings (level 9 of the hierarchy). At this point the normally developing child realizes that a certain surface form is used to signify some particular person, pet, familiar act, salient event, or whatever the case may be. At this point, the comprehension of at least one complete pragmatic mapping of an arbitrary conventional linguistic sign (the sort seen in Figure 1) is attained. As soon as this step is completed, it can be said that the child has achieved comprehension of the first meaningful word (or signed gesture). Usually, it takes several (three or four) additional months of development before the child is able to produce a recognizable variant of the surface form of any vocal conventional linguistic sign. When the production of the "first word" occurs, usually by about the time of the child's first birthday, the beginning basis for the syntactic tier of development has been achieved and the child is ready to embark on the fourth cycle of abstraction or what may be termed the level of the zero-order predicate (level 10).

By the completion of the first three cycles of abstraction (once level 10 of the hierarchy is attained), the child arrives at a limit of semantic generality where the zero-order predicates achieved by the end of the third cycle are as general with respect to their arguments as can be had (applying as they do to all possible instantiations of the logical objects referred to by them). The sign products of these first three cycles of abstraction constitute what has been termed the semantic tier of sign systems. Six additional cycles of abstraction have been sharply defined in theory beyond the semantic tier: the next three cycles after the first triad, define a syntactic tier of advances beginning with zero-order predicates, proceeding to third-order predicates which reach a syntactic limit of diminishing returns beyond which human languages simply do not go. Theoretically, it is possible for syntax to be made a great deal more complex than actually found in natural languages, but the motivation for additional complexity is lost at the vague limit where all of the commonly conceivable predicate relations can already be expressed. Also, there are limits as suggested by George A. Miller in 1956 [38] and by Peirce [3] on the number of arguments that can be brought under the scope of a given predicate-due in part to the vague (and variable) empirical limit on the number of things that we human beings can hold in mind during the time it takes to produce the complete thought underlying a conventional sentence.

The final triad of cycles produces an increasingly abstract and general pragmatic tier that never reaches a limit of complexity but does arrive at an absolute logical limit of pragmatic generality - the 
sort of generality approximating a perfected continuum, to which, according to Ketner and Putnam [39], Peirce applied the symbol omega, $\Omega$. By that designation he aimed to suggest a theoretical limit of multitudinousness where the multitude of multitudes of individuals (whatever they might be) no longer have boundaries separating them. The physical possibility of such a continuum of atoms, where the bounded icons of a given sort become so densely packed that they merge into a "super" icon-a continuum where the individual atoms become one-was empirically demonstrated by Anderson and colleagues in 1995 with supercooled rubidium atoms [40]. Their work not only won Anderson a Nobel Prize in physics but has been cited since its publication almost 3,500 times in journals indexed in the Web of Science as of today's date (February 24, 2010). Setting aside the question of whether or not Peirce actually proved mathematically that such an $\Omega$ limit of multitudinousness exists and defines continuity, the theory of abstraction suggests plainly that the multitude and complexity of pragmatic mappings theoretically attainable at the limit of the third pragmatic cycle (the 30th level of abstraction) can express (in principle, it seems) the nature of a genuine continuum. Hence, it also provides, if correctly characterized in this way a tour de force demonstration of the inadequacy of the traditional Shannon/Wiener definitions of information as applied to discursive systems of representation: The notion of a calculable mathematical value for information absolutely vanishes when the selection of any particular discursive representation must be drawn from a multitude at the $\Omega$ limit exceeding the multitude of all possible multitudes. If a valid choice could be made, its information would be infinite, and if not, its information would be zero. But, to the extent that particular valid discourses (e.g., TNRs) can be constructed, their information must be infinite in all cases according to the Shannon/Wiener mathematical definition of information. Thus, that approach to explaining the existence of such TNRs is, to put it nicely, uninformative. By contrast, the theory of TNRs, and the theory of abstraction to which it gives rise, provide a rich basis for differentiating, grading, and measuring sign systems in ways that lead to a plethora of empirically testable hypotheses.

Indeed, the predicted sequential progression of the first tier of abstracted signs, which can be relatively easily checked against early human development, holds and contains some remarkable surprises-including the discovery that normal infants can learn to read with comprehension as soon as they are able to achieve level 9 of the sign hierarchy (usually by about month 5 to 7 after birth). The predicted sequence of signs attained is also consistent with observed facts both at the micro and macro levels. Setting aside the details of the micro level — which are dealt with elsewhere [26] — at the macro level, looking across the whole spectrum of the three tiers of sign levels, it is easy to see that the macro-sequence found in empirical observations is consistent with the logical requirements (which can be read as empirical hypotheses) of the theory.

\subsection{Macro-Level Predictions Confirmed}

The logically necessary general macro sequence (all else being held equal) must (and does) fall into the requisite order of increasingly more marked systems. We may sum up the progression in the formula:

$S \pi O>S \pi \underline{O}>\underline{S} \pi \underline{\theta}>\underline{S \pi \theta}$ where the symbol ">" is read merely as "precedes in development"

The child's first meaningful signs (or words) invariably involve simple predicates of the $S \pi O$ variety (the relatively unmarked formalization of a true narrative pragmatic mapping) where an abstract 
predicate is linked through an index to a relevant and salient event-e.g., kissing, rocking, hugging, or to a person such as mama, daddy, or even a pet, or to a salient and common sequence of events such as leave-taking, picking up or putting down of the child, feeding, changing, or whatever. Common examples of "first words" thus include utterances of referring expressions such as "Mama," "Nunu" (the name of the dog), "Baba" (to request a bottle with milk), "Bye-bye," "Hot!" and so forth. This attainment follows in a strict logical sequence from the preceding 9 levels of sign systems generated by the first three cycles of abstraction. The first productive conventional sign/word/expression which typically appears by about the end of the first year after birth, will be followed first by the comprehension and later by the ability to produce and explain the successively more marked formal systems which may be designated as fictions, errors, and lies, in that order. If we require demonstration not only of comprehension but also the ability to correctly differentiate the foregoing categories the mean age of attainment for each appears to be at about 2-3 years for fictions, 3-5 years for errors, and about 5-7 years for a sharp distinction between unintentional errors and deliberate lies [see 26 for summaries and documentation of the relevant research].

Focusing attention next on the second tier of sign systems - the syntactic one - the first productive predicates to be established in the repertoire of a normally developing child are almost as tightly associated with their arguments - the objects or events they involve and to which they directly or indirectly refer - as percepts are associated with objects that are perceived. These zero-order predicates and are sometimes spoken of as "preverbal"-as if they were not true symbols; e.g., by Tomasello [41,42]. They form the stuff with which the abstractive cycles four, five, and six, will enable the building of sequences of signs that are syntactically complex to a limit of diminishing returns.

At that vague limit (level 20 in Figure 2), where the need to be understood is satisfied and tends to be matched or overwhelmed by the need to move from improvements in form to pragmatic issues about facts, further differentiation of surface form is largely unnecessary and would be counter-productive if pursued at the expense of pragmatic questions. Such a limit with respect to linguistic complexity in general is eloquently described in Danesi's interpretation and elaboration of Zipf's "principle of least effort" $[32,43]$. If we add the compulsion to achieve understanding through TNRs that are well-fitted to the facts they represent-we come to the limit on the elaboration of syntactic form as guided by the capacity to express intended meanings well. Also, children acquiring language, like rational language users in general, in conforming to Zipf's principle of least effort as elaborated by Danesi, will necessarily also tend to satisfy the essential requirements of the Gricean maxims of conversational implicature [44] - to stay relevant, avoid falsehood, and not say more than needed [his four maxims involve an unnecessary redundancy and can be reduced to the three just stated as shown in 26]. All those principles as shown elsewhere [26] can also be reduced to the two-edged blade consisting of Leibniz's principle of the identity of indiscernibles - disregarding useless distinctions, i.e., "differences that do not make a difference" which is Gregory Bateson's dictum [45] as quoted by Brier [14] (p. 24) and which is obviously the flip side of Ockham's Razor not to multiply entities beyond necessity [46]. Human infants evidently use both cutting edges of logic to avoid, or reduce, unnecessary complexities.

It should be noted that as the child progresses through the syntactic tier of abstractive cycles (see Figure 2), concerning all the distinct orders of predication, and complications of them-including 
negation, subordination, conjunction, and disjunction - it is relatively simple to prove that there are no meaningful predicates whatsoever that have no associations with particular material arguments through TNRs. All meaningful signs of any kind depend on the sorts of simple pragmatic maps summed up in the generalized and abstracted $S \pi O$ formula. There are multiple ways to show this to be necessary. No index can achieve any meaning apart from a discriminated icon. Similarly, no abstract symbol — no concept of any kind or degree of abstractness or any imaginable complexity — can have any conventional use except by being associated through some valid index with at least one material object (or many) displayed as a distinct and bounded icon. This is proved for any symbol by showing that if it were possible to completely obliterate the indexical relation, or the material icon(s) with which it might be associated, in such a case the otherwise meaningful symbol(s) would become as empty as a pure vacuum and as indistinct as nothingness itself. Therefore, some association with materially instantiated arguments (iconically represented logical objects) is required in order for any symbolic predication to have any meaning at all. A practical illustration of this complex of logicomathematical necessities can be illustrated at a low level of abstraction by trying to imagine what would become of the concept (predicate) "dance" if there were no DANCER (no material argument) or nothing whatsoever to display acts of dancing. It follows that abstract predicates with no instantiation whatsoever in any discriminated material objects would turn out to be empty of meaning.

Even the universal predicate of negation, e.g., "There is no printer attached to this computer," needs instantiation at some time in its history of acquisition with some particular negated argument-one that was formerly present and not negated, e.g., the printer that was, but is not now, connected to the computer - so that the sign user is able to generalize the meaning of negation as a predicate that has been applied first to zero-order predicates, thus enabling its generalization to first-order predicates, and so forth. Thus, again, the necessity of grounding abstract representations in associations originating in $S \pi O$ mappings can be (and has been) proved by what Peirce termed "exact logic."

\subsection{The Implicit Transitivity of the Cycle of Abstraction}

In addition to generating the multi-tiered hierarchy of sign systems consisting of 30 distinct layers, the abstractive cycle also contains the essence of the triadic character of the temporal continuum consisting of the present, past, and future.

In particular, as an object is prescinded from a former location, the former status of the object is past relative to its present status, and the path followed by its trajectory of motion defines a future status as well. Therefore, it follows by strict logic that until the infant can prescind an icon from the moving object and project it (indexically) along the line of motion back to its former location, and/or forward to a location not yet reached along its present line of motion, that infant will remain unable to comprehend the integrity of the moving object in all of its inertial locations. Also, the idea of past and future as contrasted with the present time must, in such a case, remain out of reach.

Therefore, the abstractive cycle expresses the necessary basis for the discovery of temporal sequences. It is also worth noting that the past experience of, for instance, the former location of a moving object, constitutes a memory as contrasted with the imagined future locations of that same object which must be conjured and are not associated with prior perceptions of the object in its past locations. While the images of a moving object — say, a truck passing by on the road outside — involve 
memory traces left by the percepts of the object in one or more of its past locations, the future locations of the same object along its line of motion must be conjured and have not yet been experienced. Therefore, the cycle of abstraction would lead us to predict (correctly) that normal first language learners typically comprehend present references first, then, past tense references, followed by future references, considerably later. Again the inferences logically drawn from the theory are consistent with empirically observed phenomena in child language and cognitive development and in adult sign use and discourse processing.

\section{Neuroarchitecture and Bodily Systems}

In addition to all of the foregoing applications of the theory of pragmatic mapping and its corollaries as illustrated above in child language development, there are also strong indications that the described dynamics of the most ordinary of TNRs is contained within the neuroarchitecture of the body. It can, as I will show next, be found in the essential lateralization and dominance features of the human central nervous system as well as in the gross distribution of neurons. The underlying formula for pragmatic mapping as described in Figure 1 (B) is also evident in known elements of the genetic, epigenetic, and especially immune systems. Because the neuroarchitecture of the brain is transparently associated with the pragmatic mapping process and with the theory of abstraction, it is a good place to begin in showing the essential applications of the general theory of signs in biosemiotics.

\subsection{Pragmatic Mapping in the Human Brain}

As I have argued recently, together with Stephen D. Oller and Linda Badon [47], research with so-called "split-brain" surgeries in the treatment of epilepsy, as well as complete hemispherectomies performed in cases of malignancies or life-threatening disease confined to a single hemisphere, both show plainly that the main components of the human brain, the dominant (usually left) and subordinate (usually right) hemispheres linked by the corpus callosum in normal brains, typically have distinct specialized functions as shown in Figure 3 (adapted from Oller, et al. 2010, p. 535).

In particular the $S$ functions generally fall to the dominant hemisphere which is usually the left hemisphere as is suggested in Figure 3 while complex motor functions (e.g., speaking, writing, signing), $\pi$, generally depend on communications through the corpus callosum originating in the dominant hemisphere and coordinating (controlling) the subordinate hemisphere which specializes in handling holistic sensory signs, $O$. Similarly, the subordinate hemisphere generally manages attentional phenomena pertaining to holistic sensory icons and communicates coordination and control of sensory functions, e.g., control of smooth pursuit and visual saccades, that are found at the lower boundary of volitional movements [48,49]. It also comes out in the relevant research that the dynamic pragmatic mapping functions summed up in the $S \pi O$ formula are incorporated in the neuroarchitecture of component systems as well as in the global architecture of the brain.

The massive redundancy of the role of pragmatic mapping in the neuroarchitecture of the human brain was adumbrated in Ludwig Lichtheim's classification of aphasias as summed up in his famous "house" diagram of 1885 shown in Figure 4 [50]. The symbolic, $S$, part of the pragmatic mapping formula would correspond to what he called the "semantic" or Begriffe center; the motor part of his system to the indexical, $\pi$; and the auditory (sensory) component of his diagram would correspond to 
the $O$ of the pragmatic mapping formula. The fact that his formulation still fits well with modern accounts of the aphasias affecting the left hemisphere suggests that the pragmatic mapping formula is, indeed, embedded there in the neuroarchitecture. However, sustaining and amplifying that hunch, a great deal of additional evidence has mounted up since the time of Lichtheim.

Figure 3. Pragmatic mapping embedded in the global neuroarchiteture of the human brain. (Adapted from J.W. Oller, et al., 2010, P. 535.)

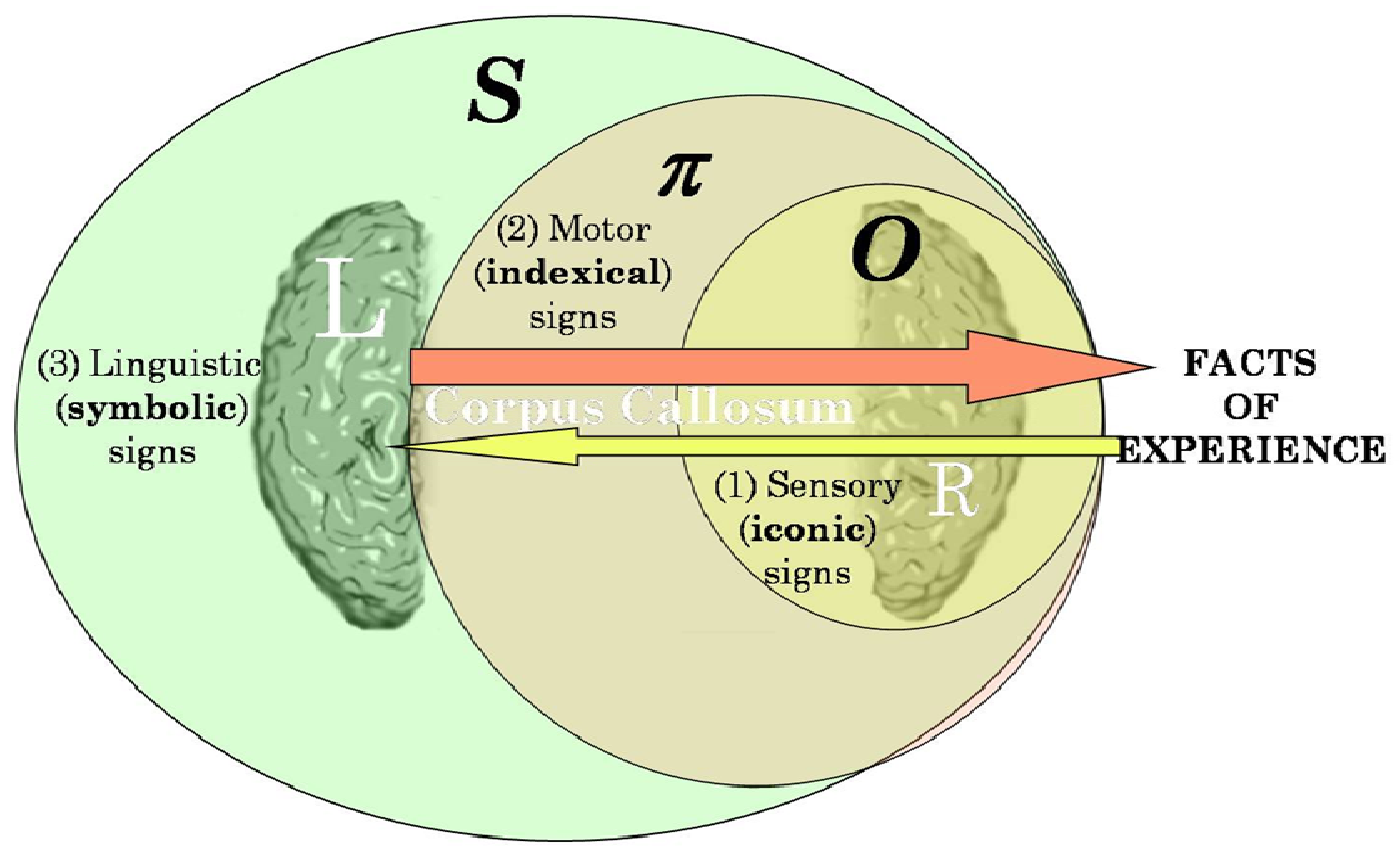

In bold strokes, the differentiation of the component systems summed up in the formula, $S \pi O$, was confirmed in many studies referred to in the Nobel lecture of Roger Sperry in 1981 [51]. Later, Sperry's findings would be reconfirmed repeatedly, and expanded on, in subsequent studies especially by Michael Gazzaniga [52]. Sketching in the distinct roles of the major components diagramed in Figure 3 and in Figure 4, Sperry noted essential distinctions in specializations of the two hemispheres and the corpus callosum, and also showed on the basis of empirical evidence that the subordinate hemisphere must also contain all but the motor command elements of the pragmatic mapping formula. The subordinate hemisphere, generalizing from what he and his colleagues showed in commissurotomy patients, could evidently perform all of the tasks except for the command and control motor functions required in intentional production of speech, writing, signing, or a carrying out a sequence of complex volitional movements. The subordinate hemisphere, however, if the dominant one were completely removed in a hemispherectomy surgery at an early age (or if the corpus callosum failed entirely to develop, as in agenesis cases), the subordinate hemisphere could evidently perform all of the pragmatic mapping functions including the conduct of complex motor functions. Sperry summed up many studies saying [51],

Each brain half . . . appeared to have its own, largely separate, cognitive domain with its own private perceptual, learning and memory experiences, all of which were seemingly oblivious of corresponding events in the other hemisphere. . . The speaking 
hemisphere [the dominant one] in these patients could tell us directly in its own words that it knew nothing of the inner experience involved in test performances correctly carried out by the mute partner hemisphere [the minor one].

Gazzaniga [52] reached much the same conclusion describing

the left hemisphere specialized for language and speech and major problem-solving capacities and the right hemisphere specialized for tasks such as facial recognition and attentional monitoring (p. 1923).

Nevertheless, in the same context, like Sperry, Gazzaniga also observed that

though each cerebral hemisphere has its own set of capacities, ... . we all have the subjective experience of feeling totally integrated (p. 1923).

At least that would be so for folks with an intact corpus callosum enabling the normal communications as well as command and control functions to keep the two hemispheres in touch with each other. There are, however, individuals in whom the corpus callosum does not develop normally or, in some cases, at all. In fact, a defective or entirely missing corpus callosum is probably more common than generally thought until recently. In any case, the peculiar difficulties seen in persons with damage to the corpus callosum are consistent with the expectations generated by the supposition that the corpus callosum is the primary conduit for sensory information flowing from the subordinate hemisphere to the dominant - as demonstrated in research with agnosias and especially prosopagnosias. The corpus callosum as described in Figure 3 also provides the main system of communications of complex motor planning and execution whereby the dominant hemisphere communicates command and control functions to the subordinate hemisphere.

An example of the latter function, one that can be easily demonstrated by anyone with an intact corpus callosum, is the phenomenon known as mirror writing. Normally it is possible for the subordinate hand to mirror the movements of the dominant hand, for instance, in writing one's name in bold strokes in the air. Note, however, that the subordinate hand is hard-pressed to perform the same task without the guidance of the dominant hand. Readers can easily demonstrate this fact for themselves by performing the experiments and then thinking through the very different impact of trying to get the subordinate hemisphere to do by itself what it can easily do with the guidance (under the control) of the dominant hemisphere. Based on such experiments, an obvious inference (a testable empirical hypothesis) from the mapping of the $S \pi O$ formula onto the global brain architecture (Figure 3) is that persons with substantial damage to the corpus callosum, and especially persons missing the corpus callosum entirely, will experience difficulty or be entirely unable to slave the subordinate hemisphere to the dominant hemisphere as seen in mirror writing by the subordinate hand. Also, we should predict that damage to the corpus callosum will induce certain disorders such as "alien hand syndrome" [53], and that complete severing of the corpus callosum, as in a radical commissurotomies, must disable the slaving of the subordinate hemisphere to the dominant one as occurs in the mirror writing function and related movements that require near non-habitual (intentional, volitional, and complex) coordination of the right and left sides of the body in complex tasks such as typing with both hands, playing a stringed instrument or keyboard, and the like. Indeed, all these predictions are borne out by relevant research [47]. 
Figure 4. The pragmatic mapping relation seen in the dominant hemisphere where damage commonly leads to any one of eight distinct types of aphasia first distinguished by Ludwig Lichtheim (1885).

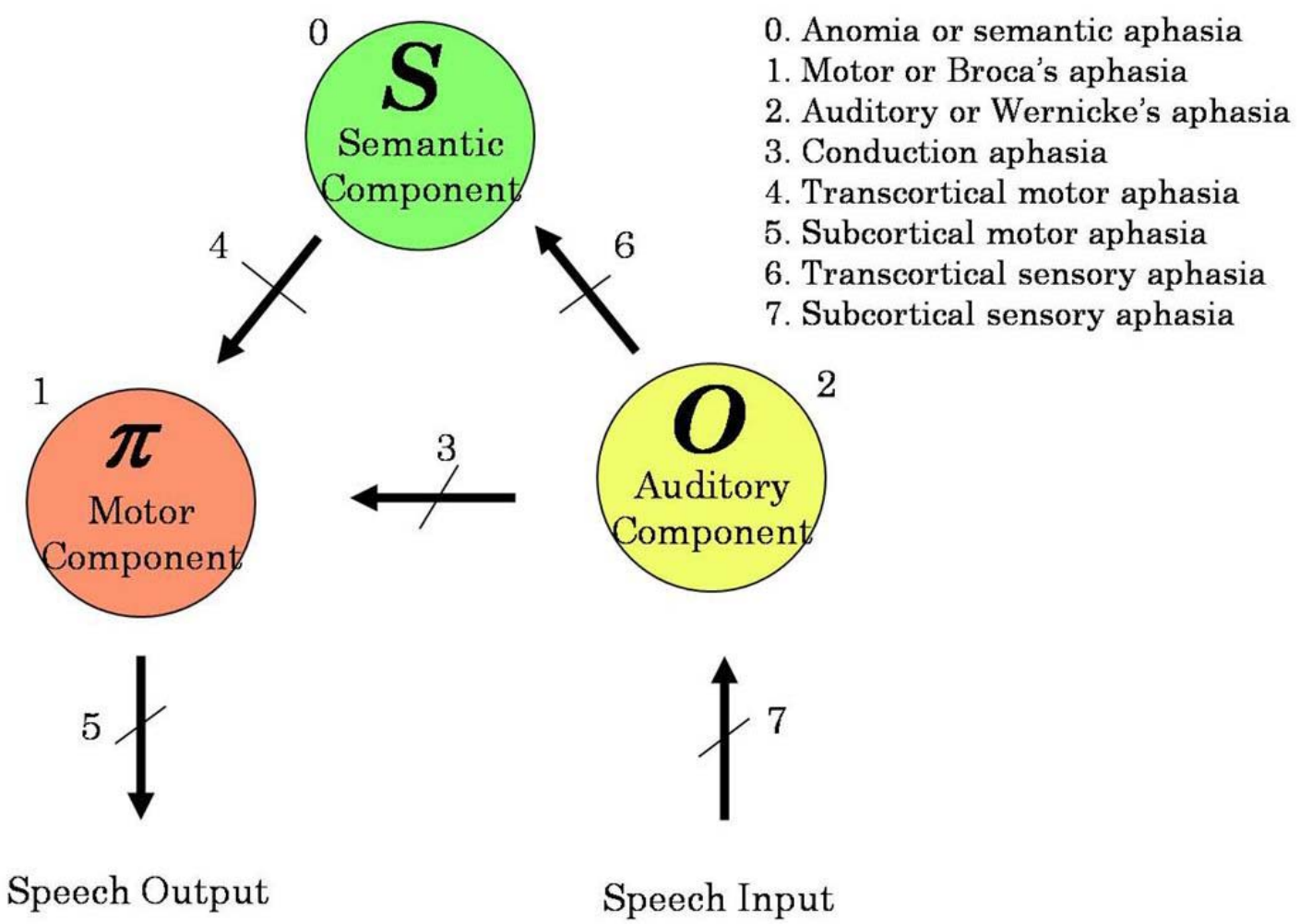

\subsection{Pragmatic Mapping in the Neuroarchitecture of Major Brain Parts}

In fact, with all the foregoing in mind, much more can be inferred from the global architecture models suggested in Figures 3 and 4. With that in mind, and with the findings from "split-brain" research and cases of complete agenesis of the corpus callosum in hand-particularly, the results showing that both hemispheres can perform all of the pragmatic mapping functions in many cases of complete agenesis and in cases where the hemispherectomy or commissurotomy occurs very early in a child's life [54-56,59] - It becomes reasonable, even necessary, to infer that the pragmatic mapping formula must describe the essential neuroarchitecture not only of the whole brain, but also of its parts.

\subsubsection{In the Parts Separately}

Figure 4 shows that the predicted organization is found in the dominant hemisphere, and by inference from cases of complete agenesis of the corpus callosum along with early hemispherectomies and commissurotomies, we know that the subordinate hemisphere is similarly organized. From Figure 4, as originally drawn out by Lichtheim, it can be seen that the pragmatic mapping formula of the global neuroarchitecture is reflected in the key linguistic functions of the dominant hemisphere. With that diagram in mind, it is clear from research with early hemispherectomies (ones performed while the brain is still developing), that the pragmatic mapping formula must also generalize to the subordinate hemisphere. Otherwise, how would it be possible to explain cases of agenesis of the 
corpus callosum [59] and/or early hemispherectomies or commissurotomies where it seems possible for the persons affected to perform all of the normal pragmatic mapping functions with either hemisphere all by itself [51,54]? The predicted architecture, again, is empirically demonstrated in both hemispheres.

\subsubsection{In the Corpus Callosum}

Furthermore, we must suppose that the development of the normal mapping of neurons through the corpus callosum must also reflect the same basic $S \pi O$ formula as suggested in Figure 5. More specifically we must predict that the rostrum and genu (the bend) at the front of the corpus callosum should be associated with symbolic functions, $S$; the body of the corpus callosum should be associated with articulate and tactically complex motor sequences (as seen in speaking, writing, typing, and signing), $\pi$; and the basic sensory functions involved in constructing icons of objects should be associated with the posterior portion of the corpus callosum, $O$.

Figure 5. The pragmatic mapping relation (in white lettering) hypothesized in the neuroarchitecture of the corus callosum (colored in green).

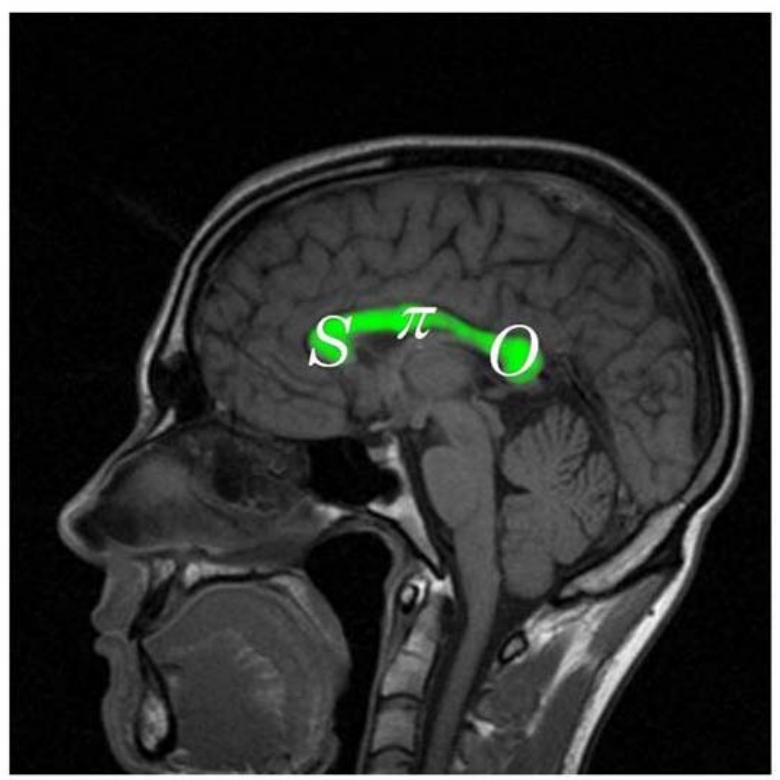

Until recently, with the development of diffusion tensor magnetic resonance imaging (dMRI) - e.g., see Paul, et al. [57] as well as Hofer and Frahm [58] - the inferences of Figure 5 could only be tested against symptomatic observations of persons with focal lesions in the corpus callosum. However, with dMRI it has become possible to map the estimated 190 million axons extending from the neuronal bodies extending from various regions of the cortex through the corpus callosum to the highest level of the neurons of the opposite hemisphere of the cortex as shown in Figure 6. The mapping actually found is indeed consistent with the formula, $S \pi O$. Also, as we should expect, focal lesions in the corpus callosum produce symptoms consistent not only with inferences at the global level of neuroarchitecture suggested in Figure 3, but also with considerably more specific predictions flowing 
from the hypothesized architecture of the corpus callosum suggested in Figure 5 and borne out in Figure 6.

Figure 6. The pragmatic mapping relation in the neuroarchitecture of the corpus callosum: axons hypothesized to serve the symbolic functions, $S$, are colored in green; those hypothesized to serve motor functions, $\pi$, are colored light blue accounting for premotor cortex and darker blue reserved for the bodily motor strip (also see the mapping of the body onto that motor strip in the left side of Figure 7); while those axons largely involved in managing sensory representations, $O$, are multicolored with red being associated with the somatosensory strip (see the right side of Figure 7), orange for the parietal Cortex known to be involved in icon construction and retrieval, purple showing the axons serving the auditory cortex, and yellow for occipital functions. Adapted and reprinted from Neuroimage, Vol. 32(3), p.991, Copyright (C) 2006, with permission from Elsevier. See Hofer and Frahm [58], Figure 1, Panel A, also [57].

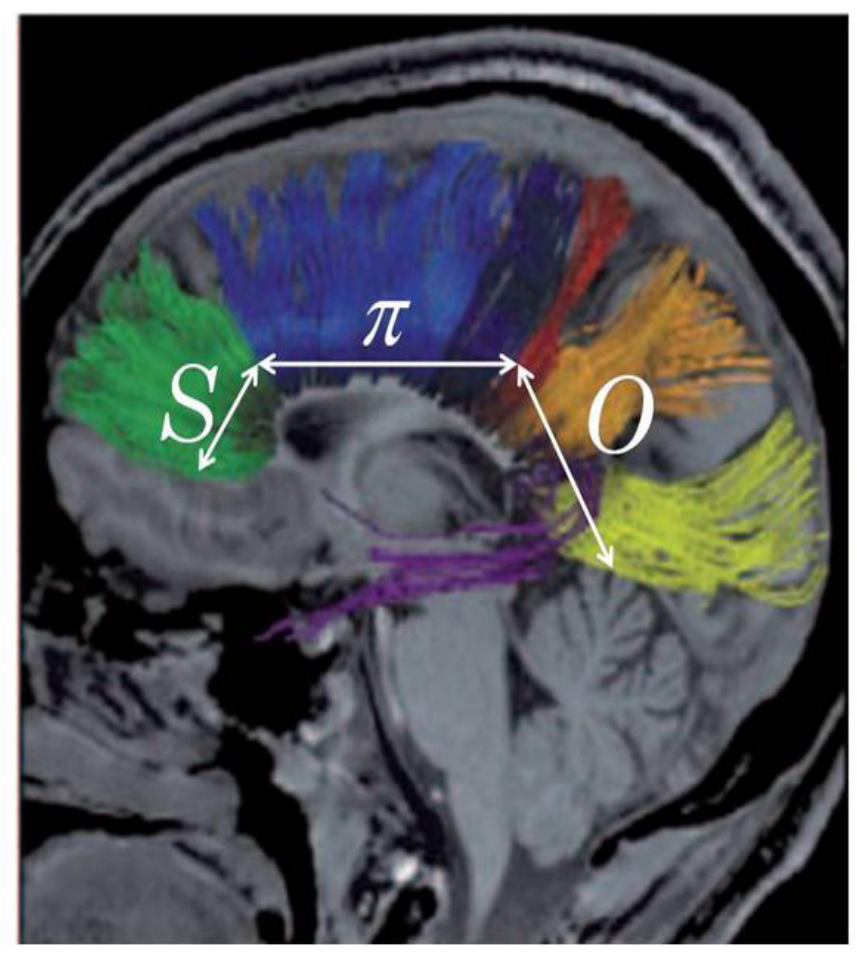

Another inference to be drawn from the foregoing observations about the corpus callosum in particular is that in disorders such as autism, where about 50\% (or more) of the persons so diagnosed are also described as "nonverbal" (meaning they are unable to produce intelligible and connected speech), we must predict that the development of whole fiber tracts emanating from the corpus callosum must either be generally stunted or dysfunctional. Indeed in 2009, work by Frazier and Hardan [63] as well as Keary et al. [64] shows that the corpus callosum is smaller than normal in persons diagnosed with autism than in matched neurotypical peers.

On a more subtle level, we can generate hypotheses concerning more specific focal lesions and their impact. For instance, it is possible to infer that damage to the temporal region of the dominant hemisphere-The region that presumably enables auditory comprehension of the naming relation, a 
supposed prerequisite to verbal production of such a naming relation-ought to interfere with the ability to name a famous pictured person while damage to the subordinate hemisphere in the same temporal region should result in an inability or difficulty in recognizing the picture of the famous person in the first place. In fact, in 2007, Gainotti reviewed research with prosopagnosia showing generally that damage to the dominant hemisphere temporal lobe resulted in inability to supply the name of a famous pictured person, while damage to the same region in the subordinate hemisphere generally involved inability to store and process the image - i.e., to know that a picture was of any particular person, famous or not, and irrespective of the name of that person [60]. Pressing the point a bit further, Anaki and others [61] report an individual with a left (dominant) occipital infarct lesion who showed difficulties in facial recognition "only when . . required to name a famous face, or select two faces from among a triad of famous figures based on their semantic relationships" (p. 1658). The individual in question "is able to select a famous face from among non-famous distractors" but is stumped if asked to name the famous person (e.g., "Michael Douglas," "Antonio Banderas," "George Bush," "Tony Blair," "Winston Churchill," "Adolf Hitler," etc.), or to select two faces that share a semantic description from a field of any three, e.g. "both are actors," "both are political leaders," or "both were involved in World War II."

It would appear that damage to the dominant temporal region commonly results in a kind of semantic/linguistic agnosia - having difficulty thinking of the name of a person who is recognized as famous and whose name is well known - while damage to the subordinate temporal region tends to produce difficulty of a conceptual sort at the level of recognition. In the latter instances the agnosia involves inability to look at a familiar face, for instance, and recognize it. The latter kind of problem describes the sort of classic and extreme form of prosopagnosia in which a person is unable to recognize even their own face in a photograph or a mirror.

Based on the empirical studies reviewed and with the predictions that can be derived from the global neuroarchitecture of the whole brain, as well as the evident redundancy of the same sort of architecture contained within the individual hemispheres and the corpus callosum, we should infer that damage to the icon producing and management components of the subordinate hemisphere, and/or to the posterior portions of the corpus callosum enabling communication of iconic representations to the dominant hemisphere must tend to result in some form of agnosia - an inability or abnormal difficulty in holistic perceptual processing, as if the conceptual underpinnings of the images to be processed were simply inaccessible or missing. By contrast, damage to the dominant hemisphere and/or to those portions of the corpus callosum enabling it to communicate articulated motor sequences to the subordinate hemisphere should result in the sorts of aphasias as described by Lichtheim and others (Figure 4) as they are, in fact, known to do.

\subsubsection{Somatotopic Organization of the Neuroarchitecture of the Body}

Focusing attention still more specifically on the massive redundancy of the pragmatic mapping formula with respect to the neuroarchitecture of the brain, consider next the iconic mapping of the body onto the sensory, motor, and premotor strips - or the so-called "homunculi" of the brain. It has long been known that the iconic representation of the body itself with respect to its parts and motor functions, as seen in Figure 7, consists of pairs of mirror image maps whereby each half of the body is 
mapped to the opposite side of the brain roughly in inverted form, excepting the face and mouth which are mapped in an upright orientation. It is noteworthy that the tongue and throat are set apart at what seems to be the highest rank of the hierarchy of conscious and volitional representational systems of the brain - roughly corresponding to the top half of the whole brain with the bottom half, more or less, devoted to autonomic functions or to ones that come under the control of the, literally, higher semiotic systems - especially those having to do with linguistic representation (note the position of the tongue).

Figure 7. The somatosensory and sensory-motor strips of the cortex. From Figure 46.9 of Life: The science of Biology 6th ed.; 2001, p. 820. Reprinted with permission from Sinauer Associates. All rights reserved.

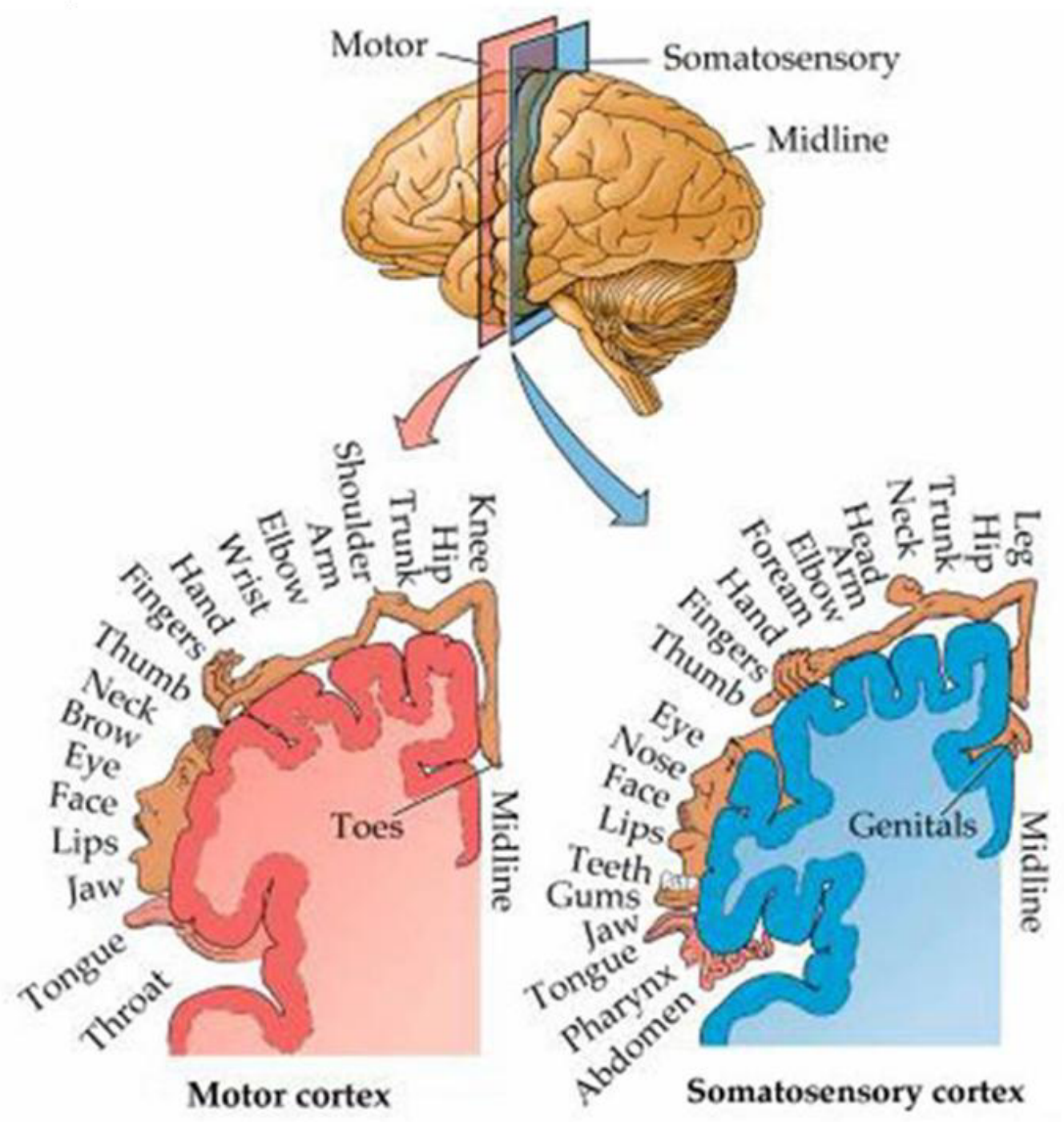

The cortical area devoted to the sensory icon of the body corresponds roughly to the region defined by the quarter spherical projection of the red axons of Figure 6, while the motor functions are similarly mapped to the cortical region roughly corresponding to a similar projection of the dark blue axons shown in Figure 6. This can be seen by comparing Figures 6 and 7. What is less well known is that a similar iconic mapping of the body seems to hold for the premotor regions defined by the lighter blue axons on either side of the brain in Figure 6 [62]. Thus, it would appear that there are three 
increasingly complex iconic projections of the whole body onto the cerebral cortex rather than merely two as seen in the traditional picture summed up in Figure 7-going back to Penfield's initial discoveries $[65,66]$ which continue to be amplified [62] with respect now to a still higher symbolic icon of the body. Indeed, it cannot reasonably be denied that the icons of the body in the cortex are all symbolic, only at different levels of abstractness, generality, and diversity of the dynamic semiotic functions served. It would appear that there may be three orders per Figure 6.

In each case, with respect to command and control functions of volitional acts especially involving the corpus callosum (compare Figures 6 and 7), the mapping of the body appears to be proportional to the diversity of functions subserved - that is, more cortical area is devoted to those portions of the body that are used for a greater variety of functions with special emphasis given to ones coming under intentional and volitional control. Referring to Figure 6, with the work of Esopenko and colleagues in mind [62], it can also be argued that there is a premotor icon involved especially in representing possible actions; a motor icon enabling intentional movements of the corresponding movable parts of the body; and there is also a sensory icon capable of reporting movements as they are carried out.

Again, we see redundant embedding in these three icons corresponding to the $S \pi O$ formula. If we think of each icon as a representative of the body's representational systems, we find the $S \pi O$ formula manifested from the top downward: at the top we find the symbolic functions of the body, $S$, as hierarchically manifested in the tongue, throat, face, and hands - the sign producing parts of the body that come under volitional control; followed by the arms, trunk, legs, feet, and toes which are critically involved in our mobility, $\pi$; and beneath all that we find the rest of the body, $O$, over which we have a great deal less volitional control. Beneath the feet and toes, in the somatosensory icon, right at what would appear to be the border between the neurological systems that control volitional and autonomic systems of the body, we find the genitalia followed by the gut. In the motor icon beneath that same border we find the gut itself. It remains to be seen, in future research, how the premotor area icon shapes up in its details. Nevertheless, it appears that the three icons pertaining to information about the body itself are arranged from front to back and top to bottom in conformity with the $S \pi O$ formula.

Not only is the brain globally organized in the manner predicted by the formulas drawn from Figure 1 (Parts A and B) at the macro-level, but in the architecture of its main parts it is massively redundant with the same organizational pattern. Likewise, the several icons of the whole body mapped into the neuroarchitecture of the sensory and motor systems also appear to be arranged in conformity with the most basic pragmatic mapping formula. Based on this reanalysis of neuroarchitecture, an analysis informed by the theory of pragmatic mapping and the abstractive cycle, we must infer that some of the prior statements about commissurotomies had to be incorrect. It is exceedingly unlikely, based on the analyses summarized in Figures 1-6 that the complete agenesis of a corpus callosum could leave all intellectual and motor functions unaffected-i.e., perfectly within the normal expected limits. Although Sperry [51] and others [59] reported on cases of agenesis of the corpus callosum which were characterized as leaving intellectual abilities unaffected-seemingly controverting the global negative impact of such agenesis predicted by the sign theory discussed here-more recent work suggests that the measures and observations of intellectual performance in the various instances of the studies just cited were probably too impoverished to detect deficits in the sorts of abstract thinking required for the comprehension and/or production of metaphor, irony, and so forth. Controverting those earlier studies of complete agenesis of the corpus callosum, and in conformity 
with the predictions of the more developed semiotic model of the human neuroarchitecture as spelled out in the foregoing discussion, several more recent studies deserve mention.

Merrick reports in detail on a case of complete agenesis with "deficits in problem solving, verbal fluency, and abstract reasoning" [55]. Similarly, Paul et al. discuss several other studies showing that in cases of complete or even partial agenesis (a missing or even partially underdeveloped corpus callosum), there is tends to be "impairment in learning and memory, executive functions, motor coordination, intellectual abilities, academic achievement, language and perceptual reasoning" [57]. Following up on that work, studies of upwards of 619 cases of complete or partial agenesis of the corpus callosum are consistent with the global predictions of the theories of pragmatic mapping and abstraction - requiring the inference that significant damage or absence of the corpus callosum can be expected to result in cognitive-linguistic deficits especially at the higher levels of processing and reasoning. It is also a virtual certainty, as spelled out in cascading effects models of disorders $[26,47,67]$, that the kinds of genetic and epigenetic issues leading to global agenesis of a neurological component as large and important as the corpus callosum will tend to be accompanied by many comorbid disorders and abnormalities in other areas and the research plainly bears out this expectation [68].

\subsection{Afferent, Efferent, and Interneurons}

At the intermediate level of individual cells of the nervous system, it is worth noting in passing that the grand classes of afferent (sensory) neurons, efferent (motor) neurons, and the ubiquitous class of interneurons - apparently including the astrocyte glial cells of the brain [69,70]-also correspond to $O, \pi$, and $S$, respectively. It is interesting also that the relative numerosity of neuronal connections is the least for afferent functions, considerably greater for efferent ones, and vastly greater for the higher symbolic interneurons. When the exceedingly more numerous glial cells, all of which seem now to have interneural functions, it is plain to see that the sensory neurons reporting about objects, $O$, though significantly outnumbered by the motor neurons enabling movement, $\pi$, all those put together are vastly exceeded by the interneurons and the glial cells that seem to be involved mainly in providing for the symbolic hierarchy, $S$, which is accounted for by a large majority of the grey matter in the deeply convoluted and thus compacted thin sheet of the cortex (not shown in Figure 6, of course, where the focus is on sensory and motor functions integrating both hemispheres through the corpus callosum).

\section{Genetics, Epigenetics, and Biochemistry}

Turning next to the micro-level presupposed by the body's cells, differentiated tissues, distinct organs, and, especially, the body's systems for protecting itself against invasive microbes and toxic chemicals, we look first to the genetic systems.

\subsection{Genetic Systems}

In general, DNA molecules can be regarded as the foundational symbolic systems of genetics, $S$; the various RNAs together with the ribosomal protein factories of which they enable the construction may be regarded as the dynamic interpreters of messages from the DNA as it is communicated to the 
cells of the body, $\pi$; and the bodily proteins built up into material systems can be regarded as the functional objects of the foregoing systems into which the foregoing sign systems are mapped to produce the protein object(s), $O$.

Again, it can be shown for each of these subsystems that they are intrinsically also redundantly organized within themselves according to the essential trinitarian system of relations. The term "trinitarian" is chosen here over "triadic" because it seems that within the dynamic pragmatic mapping relation in any given TNR each part contains the whole. It may also be noted that Peirce's original trichotomy of symbols, indexes, and icons was, according to himself, inspired by consideration of the biblical Trinity. With that in mind, it is at least plain that the foundational $S \pi O$ formula is very different in the internal unity of its well-apportioned parts from any merely mundane triadic relation. In a mundane triad, the three things that come into relationship do not necessarily represent each other, much less do they necessarily do so in anything like a relatively perfect (complete) way.

However, because of the peculiar nature of TNRs, the three elements, while retaining their distinct identities, nonetheless must agree with each other to such an extent that each, as can be logically proved, must express the whole or the TNR would, quite simply, not be true in the requisite ordinary (mundane) sense [8,71]. With that in mind, it appears that only a TNR relation would satisfy the previously quoted statement of Peirce that "the most perfect of signs are those in which the iconic, indicative, and symbolic characters are blended as equally as possible" [30]. Also, it is worth mentioning that his idea of "resonance" between the material cosmos and our capacity to understand it, at least in part, was the basis for his "neglected argument for the existence of God" [72]. Somewhat later on, Einstein would often express a similar inference, though he did not take it quite so far as Peirce. For instance, in 1936, Einstein [73] asserted plainly that the human ability to understand anything about the universe is a "miracle" (p. 61).

It seems we see a fleeting reflection of that grand miracle in each of the TNRs in functional genetic representations. For instance, there must be a valid - though certainly dynamic-symbolic representation in DNA $(S)$ without which the RNAs could not produce the ribosome factories $(\pi)$ that enable the manufacture of the requisite proteins $(O)$. To the extent that such a correspondence qualifies as a TNR (and no further), it follows by strict logicomathematical necessity that it will afford the determination, connectedness, and generalizability of that whole true narrative relationship. No object itself is determined by physical laws in the least degree, contrary to what Einstein evidently presupposed in his search for a fully deterministic (law governed) "unified field theory" [71]. But, only insofar as events can be truly represented in the mundane sense of truth-which for us as observers can evidently only happen in the manner of a TNR after the facts that we are able to observe and represent - it comes out plain as day that we always look from the $S$ through its $\pi$ mapping to the $O$. We cannot work in the other direction from the material $O$ through its $\pi$ mapping to the $S$ until and unless we already know the $S$. If we do, we are able to determine the meaning of the sign by connecting it through its $\pi$ mapping to its $O$. To offer a useful metaphorical illustration, without the grand blueprints $(S)$ enabling the construction of both the factory and the new cars that will roll off the line $(\pi)$, there would never be any assembly line or any actual cars $(O)$ coming off that line.

However, once a well-formed TNR is achieved, each part presupposes the others. The massive redundancy of the pragmatic mapping formula can also, as already illustrated in the human 
neuroarchitecture at the macro-level, be seen in the parts of representations, as well as in the whole throughout the biosphere from genetics upward.

\subsection{The Whole in the Parts}

In the DNA, that is in each genome itself, the $S$ portion consists of the whole genome-a vast library of texts containing a fairly complete (though dynamic and at risk) representation of the organism itself, $O$, as well as, the means by which to construct it, $\pi$. Within the genetic interactions, there are evidently many layers of sign systems involved - as suggested by Figures 1 and 2 per the theories of pragmatic mapping and abstraction. This is certainly evident in mapping the genome viewed as a symbol all the way to the organism viewed as a logical object. However, it is evident that the basic pragmatic mapping formula applies to the known units involved at the most basic biochemical level as mapped out in Figure 8-following the foundational claims of Francis Crick in 1958 and 1970 when he stated and then reiterated what he called the "central dogma" of molecular biology $[74,75]$.

Figure 8. An amplified variant of Crick's 1958 version (adapted from his 1970, Figure 3, p. 562) of his "central dogma" of molecular biology.

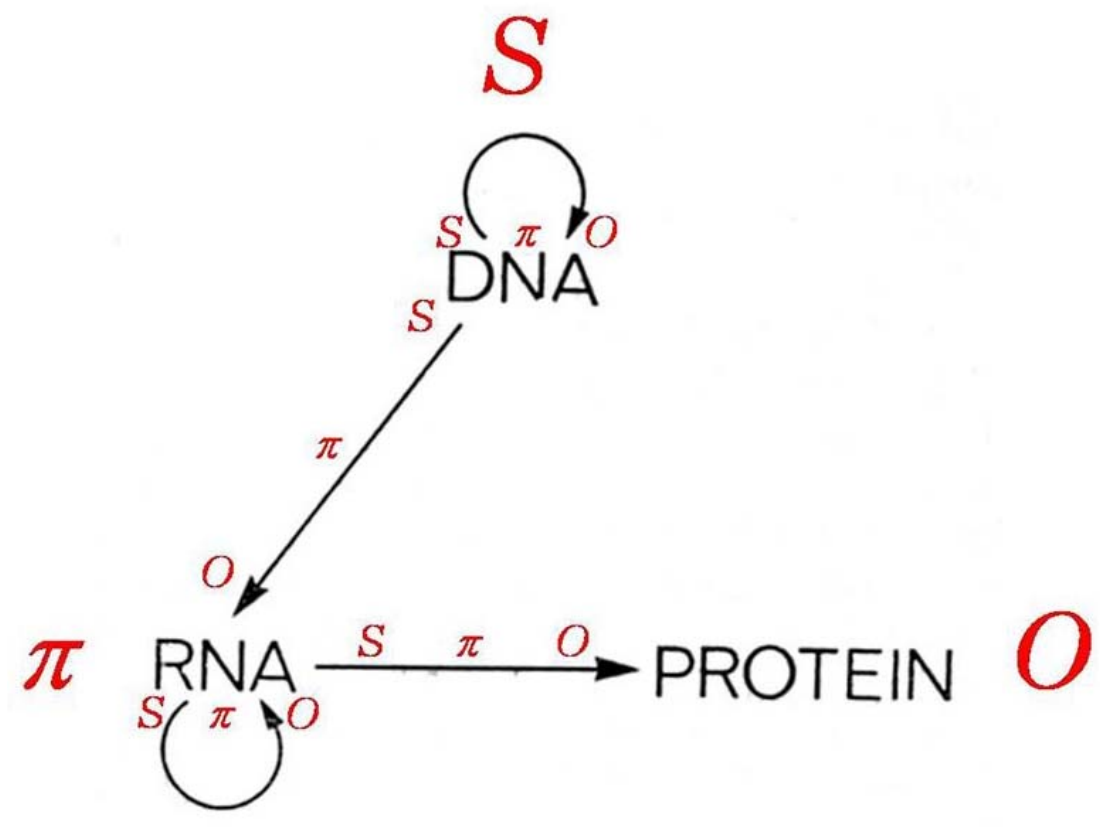

Setting to one side the controversy concerning both Crick's choice of words and the complications that would soon be revealed, it is interesting that the directional flow of the meaningful genetic determination of the organism-or what Crick somewhat inappropriately called the "genetic information" extending Shannon's inadequate term (see Brier [14]) - is consistent with certain fully abstract logicomathematical requirements on TNRs and the underlying dynamic pragmatic mapping relation. Somewhat surprisingly, the truth property of any TNR, and thus the determination of its object, $O$, is owed exclusively to the symbol, $S$, in its relation to the $O$, and not at all to the object itself. This follows by the strictest logic from the fact that without the true mapping of symbols onto 
their objects in the manner of TNRs, the determination of the difference between a billiard ball and a star or between nothing at all and anything in the universe, is quite impossible [71].

Beginning with the sugar bases, codons of three bases each $(S)$ are constructed in such a manner as to enable a mapping $(\pi)$ onto logical objects consisting of amino acids $(O)$. At a higher layer in the same grand system of representations, certain sequences of codons marked with start and stop signals constitute genes $(S)$ which can be mapped $(\pi)$ into certain sequences of amino acids to constitute functional proteins $(O)$. In that mapping process, the sequence of codons in the DNA gene specifying a particular protein system, the $(S)$, is read $(\pi)$ into a potential utterance in messenger RNA $(O)$ which in its turn serves as the $(S)$ for a sequence of anticodons in transfer RNA $(\pi)$ which correspond to certain $\operatorname{aminos}(O)$, and so forth.

In the RNA, the $S$ portion of the formula can be seen in the messenger RNA which contains the essential plan for a particular protein; the $\pi$ portion is found in the ribosomal RNA which performs the actual linking of the chain of amino acids; and the $O$ portion consists of the transfer RNA that collects aminos one by one and brings them to the ribosome to be placed in the chain. With respect to proteins, again, the system of relations appears to be exceedingly complex and layered, but each protein may itself be regarded as a complex symbol in itself, that is, an $(S)$, serving various biochemical functions, which systematically connect it through a dynamic $(\pi)$ mapping with the cell, tissue, organ, and organism $(O)$ of which it is a part.

Among the conclusions that seem to follow by necessity from such a general sign perspective is that the parts presuppose the whole, and vice versa. While it is impossible (as yet) to trace the full sequence of sign systems - from beginning to end - the existence and the holistic nature of any viable organism in any suitable eco-system implies that a valid mapping of multi-layered sign systems is not only required to account for any such organism, but that such a valid mapping must be possible. In any case, it is logically necessary that the sorts of mappings that result in viable organisms must utterly depend on the kind that can be described as "true" in the most mundane sense of that word.

The straightforward conclusions suggested by a general theory of signs also appear to be valid with respect to the genuine risks involved in the production of either the blue print - as in replicating DNA - or the line of cars coming off the assembly line - as in translating from the blue print all the way through to the requisite proteins - as seen in the functions of RNAs and the ribosomes. Plenty of risk is involved right up to the point that a valid representation is achieved. At that point, the risk is at a minimum-hence, the definition of such a theoretically (relatively) perfect (completed) representation as the antithesis of entropy. Moreover, in view of the massive redundancy of TNRs of the kind just described, in bodily systems as in ordinary experience, it follows that the means to identify and expunge errors must be implicit within any well-formed genetic system as well as within the epigenetic and biochemical systems that it subtends.

\subsection{Setting the Limits of Genetic Systems}

Between the upper limit on meaning attributed to the sort of relatively perfect system seen in a TNR and the theoretical lower limit of complete entropy, as argued earlier and as demonstrated with linguistic systems, we find fictions, errors, lies, and various gradations of nonsense verging on the 
lower limit. If the theory is valid, we should find similar gradations from TNRs verging at their lower limit toward utterly incomprehensible nonsense.

Analogues for each of these elements can in fact be found in genetic systems: genetic fictions are seen in genes that are suppressed (not turned on); errors in the sort of survivable confusion resulting in defective proteins leading in many known instances to diseases such as sickle cell anemia, Tay Sach's disease, etc.; outright lies being exemplified especially in viruses and disease agents that represent themselves as legitimate components of the body though they are not and which, if mistaken for legitimate components of the body may result in diseases and especially cancers; and, finally, we also see various grades of nonsense that may exist within DNA, RNAs, or proteins, which, in sufficient quantity, will, again, inevitably result in the demise of the organism, or, in the case of cumulative damage to the germ line, of whole species.

False representations, it would seem, generally clutter the life-space of any organism or group of them, remaining relatively benign until some threshold is reached when things really begin to go haywire. Theoretically, there is a tipping point beyond which the error ridden systems of a cell, tissue, organ, organism, or, possibly a whole community will descend into a crisis of decay and eventually complete dissolution. Along the way, there may be many ups and downs, but the inevitable tendency, as in the gravity driven dust particles observed by Pasteur in his famous experiments and lectures [76], the tendency is for other forces, in general to be overwhelmed by the prevailing force of gravity.

Mathematicized models of a general theory of signs along the lines of Ludovico [77] are theoretically possible, though modification of his starting assumptions would be required to take account of the arbitrary nature of volition which is at a maximum in intentionally producing a fictional (imaginary) representation. In fact, the freedom of volition is never entirely constrained by perceptual and/or memorial access to facts even in instances where an individual is attempting with all due diligence to produce a factual TNR. Many arbitrary choices are possible along the way with respect to what to represent and how to represent it. Also, it is a well-known characteristic of narratives in general that they can be summarized and/or paraphrased in uncountably many ways, and true narratives are not exceptional in these respects. What is more, TNRs have the added logical property, to the extent of the truth of such narratives and of the connectedness of the universe, of being infinitely expandable in principle, and of being necessarily and probabilistically connected to uncountably many valid inferences implied or presupposed by every such narrative.

Although it is clear that efforts to construct true narratives are troubled by perceptual errors, limits on competence, and by confabulatory memories - all of which also contribute to false expectations and incorrect presuppositions - to the extent that any TNRs exist, false ones, or false elements in any narratives whatsoever, become subject to the possibility of correction. Illusions and, especially, hallucinations - excepting deliberate efforts to produce them in experimental manipulations or with drugs - are generally minimal factors in generating undetectable errors in ordinary narratives.

Even in cases of extreme fatigue, stress, injury, disease, or intoxication-where the likelihood of illusions and outright hallucinations can be greatly increased-the possibility of detecting and identifying the errors in question for what they are is generally so much higher than the likelihood of mistaking them for ordinary experience that solipsists and skeptics in general who seek refuge from truth by pleading the undeniable existence of lies, errors, illusions, hallucinations, and so forth, end up looking a lot like the emperor with no clothes. In the end, true representations can reveal the rest, but 
fictions, errors, lies, and nonsense are not capable, in the final analysis, of discriminating, comprehending, and expressing simple TNRs. It is for this simple reason that the notion of negentropy as a definition of "information"- - aiming to improve it as a theory of meaning - is doomed to failure from the start. It fails to advance toward a definition of any real content for representations in the same way that the characterization of light as "negative darkness" is of no real use in efforts to determine the physical properties of light, e.g., its velocity, response to gravitation, sources, wave-lengths, etc. By the same token, for all the reasons brought forward by Brier [14], the Shannon/Wiener mathematical definitions of "information/negentropy" cannot adequately characterize the meaningful content of ordinary discourse.

\subsection{Discovering and Correcting Errors}

The surprise of discovering falsehoods - whether asserted, expected, or presupposed which are sometimes subsequently replaced by internally consistent TNRs - gives rise to the hope of achieving similar resolutions of other as yet unresolved inconsistencies. As a result, a newly discovered error may not only direct the focus of attention, but may afford the effort necessary to seek to resolve further contradictions between distinct but conflicting representations that may be conceived. Thus, the discovery of the disequilibrium of errors provides the impetus to lead advancing learners in infancy-like the little theoreticians that they seem to be as cognizant human beings-to seek out simpler, more coherent, and more valid representations of the TNR variety in which the consistency between representations and facts is relatively more complete.

When such resolutions are achieved, the result is something like the "Aha!" experience commonly discussed in the psychological literature, or what the Danish ethologist I. Reventlow, according to the translation and commentary by Brier [14], has described as a "rependium"-a neologism for sudden "insight" in which "the individual elements that are of relevance . . . are integrated into a totally new and stable structure" (Brier, 2008, p. 167). Peirce gave a similar explanation for the attainment of what he termed "the unity of conception" [78]. He wrote in 1866, "Every conception is introduced for the purpose of bringing the manifold to unity" (p. 520). In his explanation, Peirce [78] noted that "impressions (or more immediate conceptions) cannot be attended to, to the neglect of an elementary conception which reduces them to unity" (p. 519), and, then, anticipating the later analysis of Dasgupta [22] which would be written over a century later, Peirce added: "On the other hand, when such a conception has once been obtained, there is no reason in general why the premisses [sic, Peirce's preferred spelling] which have occasioned it should not be neglected, and therefore the explaining conception may abstract from the more immediate ones and from the impressions" (p. 519).

An explanation of the sort of insightful rependium being described by Peirce could involve a metaphor as noted by Dasgupta [22] in 1994-serving as "a scaffolding that could be discarded once the conclusion has been drawn" (p. 30). Indeed, Dasgupta then immediately observes in a note that he has himself just illustrated such a metaphorical representation by referring to a scaffold as a metaphor for metaphorical reference. It comes out in any such reasoning, as is brilliantly illustrated by Brier [14], following Peirce and others, that the processes of representation are invariably layered in levels embedded within levels. Such observations illustrate the essential role of recursive processes and of representations of representations that may accumulate to considerably many layers of depth. 
A necessary inference that is not always brought out, however, is that to escape meaningless and empty infinite regresses away from the real world (in endless recursions leading nowhere), it is essential to acknowledge the role of TNRs that ultimately provide the basis for all meaningful representations. All of the logicomathematical proofs concerning TNRs demand recognition of a real common world of experience for this reason. The essential conclusion is modestly suggested in the context of a discussion of a "multiverse" by Brier [14] when he remarks, "I still think, perhaps naively, that we all live in the same universe" (p. 148). It would certainly be difficult for us to agree (or disagree) with each other on anything if we did not! So, clearly we must either inhabit the same universe, or I must be strangely deceived in supposing that I agree with Brier in saying that we are in fact in the same common universe. Such a discovery, however, utterly depends on TNRs.

The homeostatic power of simple TNRs consists in their inevitable conformity to facts and in their agreement with each other. It is because of the tendency for true representations to converge on shared understanding that errors and deliberate lies can often be found out and sometimes corrected before further confusion and possible harm comes from them. Fictions, by contrast with errors and lies, are relatively less apt to lead to costly mistakes. As is beautifully illustrated in Peirce's writings and by Dasgupta [22], the conception and design of useful artifacts - possibly followed by their eventual construction and manufacture - is greatly dependent on fictional representations that are eventually transmogrified into TNRs. However, it is clear that fictions, errors, lies, and all forms of nonsense, all else being equal, must always contribute to an increase in overall entropy. To illustrate the inevitable fact that a false representation contributes entropy in the grand economy of things, consider the time and effort required to produce a representation in the first place, then to interpret and check it against facts, and finally to go back and correct it if the representation has turned out to have been mistaken. The essence of the entropy contributed by any unnecessary fiction, one that is certain to turn up false or merely nonsensical, is suggested by the rhetorical question some sage put on a bumper sticker many years ago: "If you don't have time to do it right the first time, when will you have time to do it over?" The lost time, if nothing else, contributes entropy. Therefore, the entropy added by a fictional representation consists in the added risk of falsehood while actual errors add a considerably greater increase in entropy, successful lies contribute still greater entropy (provided they are believed and thus mistaken for true representations), and increasing grades of nonsense must tend toward the theoretical limit of absolute entropy.

Barring the way to complete and relatively sudden dissolution of any material or biological system are TNRs. In fact, because of the determinacy, connectedness, and generalizability of TNRs - the logical properties unique to TNRs that enable necessary and probable inferences about transitive relations of all kinds - they have a kind of biologically redemptive function enabling wariness about fictions, errors, and lies. TNRs also stand as an immune deterrent to genetic lies (as in foreign DNA producing cancers), and are themselves lethal to lies in cases where the lies can be corrected and replaced by TNRs. Also, TNRs are the only means by which subtle grades of genetic, epigenetic, or biochemical nonsense can be detected and discarded before it can accumulate and do harm to systems that depend on valid representations.

It is a logical uniqueness in the nature of fully abstract TNRs that they are themselves indestructible having, relative to all fictions, errors, lies, and nonsense a secure and true existence that can be described as eternal. Once a true representation of the narrative kind is established, it is forever true. If, 
for example, to take what is believed to be a valid example of a TNR, suppose that my friend Nicholas Haiducek actually was born somewhere on May 4, 1940 and that he died on May 23, 2009 in Magdalena, New Mexico. Now, supposing only that this representation is indeed true (since nothing critical to the logical argument at issue hinges on the particular example chosen to illustrate it), we may ask if it had been a prediction at some time in the far distant past, say, before the universe existed, or if it should be uttered or interpreted an equally great distance into the future as a statement pertaining to the past, say, after the universe as we know it no longer exists at all, when along that vast hypothetical time line would the TNR not be true? It matters not a whit whether the present universe survives a day or forever, nor whether it has existed for only a short while, say, 20 billion years, or eternally, a TNR, to the extent that it is true at all, is so forever.

It is also evident that once any TNR is established it is also connected - at least to the extent that the universe is actually connected - to all the other TNRs that might be constructed at any time and in any place within that universe. In any event, to the extent that time, chance, and representations of them are able to impinge on material objects, biochemicals, organisms, events, relations, and whatever else may exist in the universe - the one that is presupposed, indicated, and impacted by every TNRwe must suppose that the universe is fairly continuous. Strictly speaking, it may be argued that the connectedness of the universe itself may decay over time while the abstract TNRs connected to it absolutely cannot [71] and that they themselves ensure an abstract eternal continuity to the universe rather than the reverse. To the extent that real continuity exists, in any case, if TNRs tap into it, it follows that TNRs are connected to each other. By the way, I think that the best representation of Peirce's views on the nature of continuity, a problem to which he devoted a great deal of attention, may be found in his 1892 paper in the Monist intriguingly titled "The Law of Mind" [80]. I say this is intriguing because Peirce, unlike Einstein, and many others, did not attribute the continuity directly to the material universe, but rather to its conception by the mind. TNR theory also confirms that this attribution is necessary.

In any case, as a result of the nature of any such continuity wherever it may reside, the inevitable long-term tendency, as argued by C. S. Peirce [2,72] with respect to scientific study, is for true representations to survive because they are true while fictions, errors, lies, and nonsense must either be converted to TNRs by correction of whatever errors they contain, or, in the end, they will lead to death and dissolution with algebraic certainty.

\subsection{Barring the Way to Errors}

Among the evidences of the body's capacity to block cascades of harmful errors at the epigenetic level of its biochemistry is a recently discovered phenomenon involving the amino acid methionine. Methionine is critically involved in detoxification owed to various chemicals - especially the sorts of toxicity produced by heavy metals such as mercury, lead, and so forth which are especially involved in fouling biological processes right down at the level of the electrons that are associated with the outermost orbitals of highly energetic and chemically reactive atoms. Heavy metals are particularly offensive in producing oxidative stress. This undesirable condition arises when the number of unattached but highly unstable ions that are extremely reactive with other biochemicals - such as the proteins crucial to the body's cell structures, metabolism, and immune defenses-becomes excessive. 
Interestingly, the research leading Morris S. Kharasch in the 1930s to understand and elucidate the nature of free radicals involved a form of ethyl mercury that was subsequently used as a preservative in vaccines [81] - the recently controversial substance known variously as Merthiolate, thimerosal, and thiomersal produced by the pharmaceutical manufacturer Eli Lilly [for discussion see 67]. The emerging theory free radicals was important to the development of the most widely accepted present-day understanding of mortality as well as the elucidation of chemically induced genetic mutations. For his ground-breaking work, Kharasch was praised highly by his fellow chemist and biographer, Frank Westheimer [82], who noted that Kharasch received the Presidential Merit Award in 1948 for his services to the Chemical Warfare Service during World War II. Recently, an epigenetic phenomenon involving methionine suggests one way the body evidently protects itself against potential damage by free radicals and toxins by adding something like armed guards to its proteins as they are in production.

The epigenetic process in question, described in 2009 by Netzer et al. [83], involves the addition to the amino acid chains constituting bodily proteins of a large number of what they have somewhat misleadingly called "regulatory errors" (pp. 522ff.). It comes out, however, that these "errors" are probably insertions that in fact prevent errors rather than constituting them. By examining what they have learned so far, we find that the body's defense and detoxification systems are evidently capable of midcourse corrections owed to information concerning toxic threats from a variety of sources including the kind produced by attenuated viruses or bacteria in vaccines, or live viruses from any source, bacteria, or many different sorts of chemically reactive oxygen species producing oxidative stress. The latter may include heavy metals such as mercury, adjuvants such as aluminum, and the adventitious agents consisting of animal protein fragments inadvertently incorporated into vaccines during their manufacture.

\subsection{Methionine Guards?}

Among the 20 major building blocks of proteins, methionine has some interesting chemical functions and properties. It is one of only two of the 20 basic building blocks of proteins that contain sulfur; it is also one of the "essential" amino acids - ones that cannot be manufactured by the body by transforming other aminos; and, it happens to be the amino used as a start marker for genes. Because of its sulfur content which can effectively grab on to various metals and toxins, methionine is naturally involved in biochemical cycles crucial to detoxification and reduction of oxidative stress [84]. It is the processes of detoxification - which are closely allied with immune processes - that the epigenetic insertions of methionine molecules seem to facilitate.

The allegedly "erroneous" insertions-as described by Netzer et al.-occur at the level of translation from messenger RNA after it has been delivered to the cytoplasm from the cell's nucleus. The researchers refer to these extra insertions of methionine as "regulated errors" but the insertions appear even to Netzer et al. to be rather more like deliberately placed armor to protect against the chemical hazards of free radicals that are already present in the cell and that might damage the proteins that are under construction and render them dysfunctional.

It is interesting that the placement of the "extra" methionines appears to be triggered by communication after the messenger mRNAs are already in the ribosome and while the tRNAs are out 
collecting their amino cargoes to bring them to the ribosome where the proteins will be constructed. When the cell is under stress by toxic biochemicals - which may come from various sources as Netzer and colleagues verified - the theory proposed by them is that toxins already present in the cell damage tRNAs which then find and deliver the wrong amino acid, which just happens always (according to their findings) to be a methionine molecule - the sort known to be crucial to detoxification and the reduction of oxidative stress. According to their theory, the damaged tRNAs not only succeed in capturing and delivering to the ribosome up to 100 or even 1,000 times the number of methionine molecules called for by specifications in the mRNA, but for some unknown reason, the ribosome accepts the delivery and places the extra methionine molecules in what appear to be "random" unexpected locations producing what Netzer et al. have called "regulatory errors." However, they see these erroneously placed molecules of methionine (Met) as beneficial and they surmise [83] that "Met-misacylation functions adaptively to increase Met incorporation into proteins to protect cells against oxidative stress" (p. 522).

The surprise is that "in vitro" experiments show a much higher fidelity of RNA translation into proteins with observed "error" rates ranging from about one per 10,000 to one per 100,000 in linking the mRNA codon with its respective amino. Although Netzer et al. and others $[85,86]$ had long supposed that error rates would be higher in living cells [87], the rates of up to 100 or even 1,000 methionine "regulatory errors" in living cells observed by Netzer and colleagues were noteworthy. Although they have tested only 6 of the 20 aminos normally involved in protein construction, so far only extra insertions of methionine have been found at the epigenetic level. They have 14 other aminos remaining to be examined.

In the meantime, it is interesting to note that the peculiar motivations of living organisms to protect themselves against toxins, viruses, and potentially harmful microbes of all sorts, seem to be evident in the deeply layered and richly interconnected hierarchy of immune systems that are increasingly being shown to depend on messaging systems that make policing and industrial-military systems appear clumsy and inefficient by comparison.

\section{The Human Immune Defense, Repair, and Clean-Up Capacities}

With reference to the human body's immune defense systems, police/military analogies are commonly used and are obviously relevant sources for a great many metaphors that can be appropriately applied as abstract descriptors of one or another aspect of the systems involved. What is not so obvious, perhaps, is that the whole body along with all the systems of the individual organism are not the only ones that must be represented. Of course, that much is obviously required in advance in order for the construction, maintenance, defense, and repairs of the body to take place, but in order for the organism to be viable, the entire representational process must succeed in a large measure in anticipating the place of the organism in its life-space along with the means for it to maintain itself and survive. As may be inferred from Brier's masterful elucidation [14] of ideas from luminaries of ethology (especially see pp. 147-173), it is clear that the genome must somehow express a theory of the particular ethological niche of the organism that it specifies. That is, the genetic plan must not only describe the individual organism itself, but must do so in a way that makes it fit viably into its 
life-space - along the lines of Jakob von Uexkull [88] — so that it may be able to survive and even thrive.

It is therefore reasonable to suppose that what was formerly called "Junk DNA" will turn out to be critical to the valid representation of the vast diversity of the life-space that is requisite to the viability of the organism as grounded in the validity of its representations. Objecting to the prevailing "dogma" of genetics, in 2009, Pellionisz [89] has argued that speculations about "Junk DNA" have "slowed down the advance of sound theory of genome function, as far as information science is concerned, for half a century" (p. 348). In the short-term, it is not unreasonable to view the body of an individual as if it were self-contained - a bounded entity from its conception, through its embryological growth and development to maturity, reproduction, and eventually its inevitable individual death. However, on a larger scale, the individual organism shares its time and space with a population of diverse other organisms great and small, all in an environment that is not without risk. The capacity to prevail against the odds, presupposes a representational system from the start that goes far beyond the mere characterization of the body as a bounded material entity. A more adequate representational (semiotic) view of the organism - one taking account of its elaborate requirements for sustenance, defense systems, repairs, and so on-must also take account of interactions between the body and its indefinitely extended life-space. Hence, Brier's argument that an ethological view is necessary must, I believe, be taken to heart.

With that in mind, as I am about to show, it is not difficult to see also that the construction of the body itself conforms in its general outlines to the pragmatic mapping formula and its elaboration in the theory of abstraction (summed up in Figures 1-7 in this paper). The body's own boundaries are not ultimate for reasons amply provided by ethologists and by Brier, yet they are nonetheless real in their own right and mark off, in the case of every individual, an entity that seems to contain that individual's self - an extreme particularity, a unique identity that represents the common world along with itself and others like and unlike it in that world, and that wonders at its own self-consciousness and the possible extent of its shared consciousness with others. The body's boundaries - the skin and internal body surfaces - define an icon $(O)$ of a particular sort. The animate history of actions and interactions with the world outside itself — seeing, hearing, smelling, touching, tasting, drinking, eating, excreting, embracing, repelling, learning, etc.-prescind the body $(\pi)$; and, its unique connections and interactions with the world represent a distinct and defining history rendering each bodily story as a unique narrative symbol $(S)$ - the whole of which may be said to represent the self in question. With that in mind, consider the application especially of crucial TNRs and abstractions that are necessary to the normal functioning of the immune systems of such an individual.

\subsection{The Definition of the Bodily Self}

The definition of what is and what is not self is the foundational premise of bodily defense. The determination of an individual bodily self begins with the completion of the fusion of a sperm and egg. Prior to that fusion, both sperm and egg have been formed by the process of meiosis in the germ cells of each parent. In that process, the female ovum and the male sperm have each received a genetic payload consisting of half the genetic material required to specify a complete (viable) individual. Each sperm cell is itself a symbol $(S)$ manifested mainly in its unmated haploid DNA as it races toward is 
intended object $(O)$ in the female ovum. Its line is an as yet incomplete indexical sign $(\pi)$ that is manifested chiefly and vigorously in the flagellum loaded with the male mitochondrial energy producers that hasten the sperm on its journey. The sperm is something like a do or die male marathon runner competing for a life-saving marriage with a female counterpart. The female gamete contains the prize consisting of the haploid DNA that can, if it is successfully fused with the haploid DNA from the male sperm, produce a viable diploid zygote. If no such union occurs, both of the haploid DNAs will be short-lived (nonviable) fictions that fail to materialize as any individual. For its part, however, the female haploid is the logical object of the sperm's race and a successful fusion between the two haploids provides the basis for the new life of the resulting zygote, a completed TNR, which is expressed as a developing embryo.

\subsubsection{May I Have This Dance for the Rest of My Life?}

For its own part, the female ovum is also highly active in screening the potential suitor sperm cells that arrive at its border. Evidently, a conversation takes place at the border of the ovum in which each sperm cell must present his bona fides. First, he must show that he is of the right kind, a suitable and desirable mate [90]. Second, he must ask for the ovum's hand in marriage as it were in order to be admitted through the wall of the ovum. Many would-be suitors, it seems, are rejected. However, to the lucky guy who is accepted and to whom the door is opened, the newly united couple agrees to close and bar the door ever after his entry so that no other sperm cells can enter the ovum and compete for the conjugal rights of the marriage [91,92] (especially p. 238 of the latter).

\subsubsection{The Ultimate in Nested Reciprocity}

The validation of the haploid payloads of DNA is evidently contained in the agreement achieved in the mating of them into a whole. The agreement in the case of double stranded DNA consists of something analogous to the fitting together of male and female parts so that a complete union is achieved. To borrow a phrase from Wagman and Miller, and at the same time to expand its meaning, here we have the quintessential example of a completely "nested reciprocity" [93] — that is, assuming only that the union is normal and results in a healthy baby at the end of a successful pregnancy. This application of the phrase "nested reciprocity" expands on the meaning of the Wagman and Miller, I believe, because they seemed to be viewing the environment as providing the peculiar energy needed to specify its representation by the organism. Yet, in the union of haploid DNAs of sperm and egg, we see something very different: in this case complementary haploid, incomplete, representations, about six billion of them, align themselves to mate and complete a nested reciprocity where approximately three billion couplings of just four sugar bases are joined to form a unique individual result. Those couplings are not determined by the environment any more than placing a haploid DNA molecule next to a rock would make it turn into one.

To some it might seem to be stretching to suggest that the DNA haploids might find something like happiness, a tingling joy, represented in their couplings. Yet, the idea of a resonant perceptual recognition within molecules of such grand complexity as seen in the three billion pairings of a single human DNA molecule may be an idea worth thinking through. It is a notion that can give new meaning to the peculiar value in the sort of personal name, a unique identity, that seems to arise in the 
case at hand. Grounding such an abstract biosemiotic notion may not be so difficult as it might seem at first.

Bringing the whole discussion down to the level of a fungus or bacterium, in a 2009 paper, Klinkert and Narberhaus [94] have argued that microbes are capable of detecting and representing temperature changes through molecular thermal gages of which the "primary sensors include DNA, RNA, proteins and lipids" (p. 2661). Supposing that this is so, it would seem that there really is something analogous to chemical perception at the cellular level as implied by Wagman and Miller [93]. If that is so, we might ask whether the detection of a conspecific haploid DNA molecule with which to mate might not generate a certain resonance on both sides. If so, could this resonant nested reciprocity constitute the very essence of the self and its distinct self-consciousness? Having put the question, I cannot pass on without virtually sensing the tingling of the qualia - to borrow a Peircean term well-deployed by Brier [14] - to suggest that biomolecules incorporate feelings, an experience of consciousness where it seems that "deep calleth unto deep" from within very small but similar living beings.

\subsubsection{Symbolically Suited for Each Other}

At each place along the chain of bonded base pairs, the union of the complementary parts confirms the sequence in both directions - with the right hand direction washing the left hand, so-to-speak, and vice versa. Each linked base pair can be construed as a well-fitted $S \pi O$ relation such that the symbol is mapped onto its object in the manner of a percept fitted to the thing perceived. As noted in the first published presentation of the theory of abstraction [8], it is logically necessary that at the highest level of abstraction, symbolic relations of the TNR variety are evidently uncountably many. That is to say, the inferences that flow from symbols toward the material world also unite the valid symbols themselves into what appears to be a seamless continuum. However, from any given observer's point of view - or from the vantage point of any given symbol which may itself be construed as a point of view - the valid inferences that flow from TNRs always yield the highest rank to the most abstract symbols, or when the abstractness of any potentially competing symbols is the same, the prior symbols dominate the ones that can be inferred from them. In general, dominance always accrues to the symbol rather than the object (or objects) in the material world with which it (or they) may be connected. Therefore, truth value (together with determinacy, connectedness, and generalizability) are in the final analysis exclusively the property of abstract symbols and never the property of the subordinate objects to which they may be attached.

\subsection{Symbol to Object, Genotype to Phenotype}

The direction of the design information flows from the $S$ through $\pi$ to the $O$ by logical necessity - a necessity hypothesized to fit the relevant empirical facts according to Crick's controversial "central dogma" of molecular biology [74,75] — see the diagram given here as Figure 8. The controversy about the central dogma has centered on questions about the extent to which the sequence and stereoscopic arrangement of amino acids in particular proteins - the acknowledged building blocks of cells, organs, and whole organisms - could be sufficiently specified in DNA [95,96].

Among the particular objections to the central dogma addressed by Crick in 1970 [75] were early speculations by Howard Temin about how cancer producing viruses could modify a cell's nuclear 
DNA so as to cause its subsequent out-of-control (cancerous) metastatic reproduction in competition with normal cells, tissues, and organs as guided by the body's original DNA [97]. Other problems that would come to light were the existence of disease agents consisting, seemingly, of nothing but badly formed proteins that seemed almost to replicate themselves-leading Prusiner in 1982 to propose the term prion as a portmanteau constructed from "proteinaceous infectious" matter [98]. Best known examples today of multiplying prions include the ultimately lethal beta amyloid plaques of mad cow disease which in humans is known as Creutzfeldt-Jakob disease, and, perhaps also the similar plaques in the brains of persons with Alzheimer's disease. In addition, there were complications in the transfer of information from RNAs to proteins and in the necessary stereoscopic folding of the latter to form fully functional variants.

The hypothetical action of viral RNA in rewriting some portion of cellular DNA was demonstrated with the independent discovery of reverse transcriptase by Temin and by David L. Baltimore in 1970 $[99,100]$ - a finding that would make Nobel laureates of both of them in 1975. It would also provide — according to some, e.g., see Morange [101,101], Rosenberg [104], and Stotz [103]—a basis for complicating the "central dogma" of molecular biology and for making it, superficially at least, more compatible with another underlying doctrine - an as yet to be determined variant of Darwinian and/or Lamarckian evolution - that would absolutely require some means of getting the information about sequences and arrangements of aminos to flow from nonliving matter backward, as it were, in order to inform DNA of its teleological functions with respect to organisms and the biosphere. Rephrasing the problem faced by the apparent incompatibility of Crick's "central dogma" with the requirements of the reverse flow of biological information from any theory of emergence or evolution in the terms of a general theory of signs as suggested by Figure 8, the dominant $S$ in the foundational sign relation, $S \pi O$, would have to be temporarily subordinated in some manner, or made a co-equal with the $O$ of that relation.

To get that to happen, the critical directionality of the $\pi$ mapping in the formula would have to be reversed. Logically speaking, this is difficult on account of the fact that the relation between any determining $S$ and its $O$ is both abstract and arbitrary. Whereas two abstract $S$ s can implicate and presuppose one another simultaneously in a completely general manner, as suggested in the recursive association of DNA with DNA as seen at the top of the diagram of the "central dogma" in Figure 8-that is, DNA (also RNA) can serve as its own object of representation - conceiving of a means by which raw aminos could form up into the many proteins required for even a bacterium or fungus, never ming how they could generate RNAs which might then be transformed into DNAs, remain as unsolved mysteries. Also, the central dogma as originally formulated would seem to preclude any means by which randomly formed amino sequences of the appropriate chirality for the proteins of a life form could operate in the "backward" direction to produce RNA and/or DNA representations of themselves.

Because the prior teaching of evolution — as noted by Carl Woese in 2001 [105] — seemed to require modifications of Crick's "central dogma" of molecular biology in order to allow for backward flow of information from proteins to RNA and from RNA to DNA-retroviruses held out hope for the reconciliation of the conflicting claims - e.g., along that line see Hoyle and Wickramasinghe in 1991 [106], also Forterre in 2006 [107]. However, the hope for making viruses the engines of DNA 
production seems to reach an impasse at just the point where the retroviruses are most critically needed as pointed out in 2009 by Forterre and Prangishvili [108]. They wrote:

... structural analyses of capsid proteins have revealed that at least two types of virions originated independently before the LUCA (the Last Universal Cellular Ancestor). Although several hypotheses have been recently proposed to explain the origin of viruses, the emergence of virions, as a specific mechanism for gene dissemination, remains unexplained (p. 466).

A dual common problem, it would seem, for each and every one of the objections raised against Crick's central dogma is that the suspected means of reversing the flow of information from proteins to nuclear DNA is that all of the modifications seem only to impact DNA outside of the germ line and are limited in their scope and/or are harmful or lethal to the organism in which they occur. Lethality, of course, is a detriment to the possibility of reproduction, but even if reproduction occurs, unless the modified DNA is in the germ line, even if the modification itself is not lethal, neither can it be passed on. The problem was well put by August Weismann [1834-1914] in what is commonly referred to as the "Weismann barrier" - the principle that genetic information flows from the germ cells to the rest of the body but not, at least not normally, in the other direction.

The Weismann principle [109] - that the "essential nature of the germ-cell dominates over the organism which will grow from it” (p. 103) — came near anticipating Crick's central dogma. With respect to the latter, Morange [101] concluded that

the only way to justify its existence [that of the central dogma] is through the description of the evolutionary history that shaped the relations between DNA, RNAs and proteins (p. 247).

\subsection{Individual Selves Consistent with Weismann's Barrier and Crick's Dogma}

Even at the level of a complete DNA molecule with its roughly three billion nucleotide pairs the mating of the two strands of the double helix in its most common form reveals an $S \pi O$ dominance relation. This can be seen in the fact that in its replication phase, the older antiparallel strands of DNA, determine the sequence of the newly transcribed strands that will be used in cell mitosis to produce the basis for cell division. Likewise, in daughter cells, that same dominance relation is maintained throughout the growth and development of the organism. The older dominant $S$ string gets $\pi$-linked through its several billion hydrogen bonds to its antiparallel counterpart during the fusion of male and female haploids after meiosis and prior to the first mitotic division which will result in the first growing phase of the newly formed zygote.

During the initial fusion (at fertilization), perhaps it can be argued that the larger sugar bases, the purines (Adenine and Guanine) dominate the smaller ones, the pyrimadines (Thymine and Cytosine). In their chemical mating it be argued that the GC pairings to form a triple hydrogen bonded nucleotides are intrinsically stronger than (and therefore dominant over) the AT pairings with their double hydrogen bonds. If so, the $S \pi O$ formula can be applied to every nucleotide as well as to the mated sequences of the old and new strands of every completed DNA molecule in every cell as implied in the highest $S \pi O$ in Figure 8. Presumably the same sort of analogy applies within the 
organelles inside each cell with respect to its parts, especially within the mitochondria. On a larger scale, taking the cell as the unit of interest, the nuclear DNA $(S)$ would seem to dominate the mitochondrial DNA $(O)$ through RNA communications connecting them $(\pi)$.

Within the DNA itself, the layeredness of the $S \pi O$ relations seems analogous in many respects to the layeredness of the human neuroarchitecture and the higher representational systems with language at what appears to be its upper limit. However, the deceptive simplicity of $S \pi O$ nucleotide pairings belies the virtually inexhaustible layers of meanings contained within the vast sequence of signs in the story to be unfolded. Also, as revealed in recent studies of epigenetic processes with multiple alternative proteins being specified by a given gene [101] and with alternative means of expression depending on available environmental factors/resources, it appears that the development of the individual, e.g., especially the immune system, comes partly under the control of exogenous factors as explained by Grindebacke, et al. [110], which must include, in the case of human beings especially, outcomes determined in part by volitional choices (e.g., ones involving diet, rest, exercise, and the like).

\subsection{The Developing Individual}

In the case of the initial DNA mapping of any given individual, the symbol and its object are almost indistinguishable except for the fact that in every pairing there is evidently a dominant and subordinate member. By convention we suppose that the dominant member is the symbolic element and the subordinate is the object. The mapping, at the level of the initial DNA, consists of the bond between the haploids with the dominant member of the pair on the strand that determines the "sense" rather than the "antisense" - and during the first mitotic division the prior strand in each case will be the dominant $(S)$ and the derived strand the subordinate $(O)$. When the validation implicit in the successful mating of the complementary halves of the double helical DNA is completed, the unfolding of the life story of the newly formed individual begins to be expressed with mitotic division.

In the process of the union of sperm and egg, the sperm's outer wall and organelles, including the mitochondria of the sperm [111] are dismantled possibly for reuse or disposal. For this reason, the father's mitochondrial DNA, except possibly in very rare instances [112], plays no role in the mitochondria of the offspring which, in effect, receives all of its mitochondrial DNA (mtDNA) from its mother. Although some researchers have claimed mixing of mtDNA from both parents, Bandelt and colleagues have argued that all such reported observations can be attributed to errors in data analysis [114,115]. Setting the possibility of mtDNA inheritance from the father aside, as soon as the first complete DNA is formed, it replicates to provide for the formation of the first two stem cells of the new organism. These cells are termed totipotent because, assuming they are well formed, they have the capacity to authorize the development of every kind of cell in the new individual [116].

In doing so, they must communicate with the host cells identifying themselves, presumably, as friend rather than foe. It seems likely that they do so, in part at least, through the common mitochondrial DNA shared between mother and child (the father's mitochondrial DNA having been eliminated as noted). Fallarino, et al. [113], in any case, showed that mother's cytotoxic immune cells that might otherwise attack the cells of the developing embryo are largely neutralized by chemical factors (cytochrome $\mathrm{C}$ and caspase-8). It may well turn out that indeed the mtDNA is a key factor in 
helping to resolve what has been called a paradox of immunology [117] — namely, how is it that the maternal immune system does not attack and destroy the developing cells of the embryo? To avoid such destruction, the developing individual must in some way engage in effective biochemical cross-talk with its mother.

Over the first four days after conception, the initial pair of stem cells will divide to 4, 8, 16, and then 32 cells to form a sphere known as a morula. The sphere will then differentiate itself by the fifth day into a blastocyst. A particular part of the surface of the blastocyst known as the trophoblast will first migrate down the fallopian tube in which it was first formed to the uterus where it will attach itself to the inner wall. At the point of attachment it will begin to form that part of the placenta that will develop into the umbilical cord and the thickened multi-layered sponge-like portion of the placenta enabling it to bring nutrients to its three distinct components: the outer layer of cells at its surface that will become the placental sack in which the embryo itself will develop, the inner area known as the blastocele containing a fluid consisting mainly of globin and nutrients that will eventually be incorporated in a yolk sac, and the pluripotent cells within the container and developing outward from its inner surface within the inner chamber of the blastocyst known as the embryoblast.

As the embryo continues to develop, after the blastocyst has implanted itself to connect with the uterine wall, the embryo will undergo the transformation known as gastrulation-a term that refers specifically to the development of the stomach and insides of the individual. While gastrulation is occurring in the developing embryo itself the blastocyst is transformed into a gastrula with multiple chambers. At the junction with the uterine wall a complex multilayered connection, communication, and circulation system is developed, including the umbilical cord and differentiated tissues of the placenta, that will provide the developing individual with access to its critical supply of oxygen and nutrients coming from its mother while also providing separation from its mother's blood and from potential attack by its mother's own mobile immune cells circulating in her bloodstream.

As it is being transformed into the larger and more complex gastrula, inside the blastocyst beneath its upper chamber that is developing into the placental circulation system and umbilical connection, the inner cell mass, now shaped roughly like a saucer thicker at the middle than at the edges, is differentiating itself into three distinct pluripotent germ layers. The disk will rotate, elongate, and divide itself along a center line while at the same time folding inward as it forms the tube for the spinal cord and its surfaces connect as its inward parts begin to form along with the head and limbs of the individual [118].

\subsubsection{The Ectoderm}

The first of the three main pluripotent stem layers in the disk shape that will be transformed in gastrulation is referred to as (1) the ectoderm, or outer layer, that will develop into the skin, the lenses of the eyes, the connective tissues of the head, the face, lips, mouth and tongue, as well as all of the nervous system. The ectoderm provides the cells needed to form the head and brain as well as the openings for the eyes, ears, nose, and mouth inward as far down as the pharynx and larynx, and from the outer skin inward to connect the openings to the urethra in both males and females and the labia of the vagina in females, and of course the anus. 
The ectoderm also contains the germ cells that will form gametes (the reproductive cells critical to the survivability of the species) as well as those that produce the "neural crest" which is sometimes regarded as a separate pluripotent germ cell line that develops into the brain and nervous system. In terms of the pragmatic mapping formula, it can be argued that the ectoderm as a defining element of the entire surface and the future of the organism as a progenitor can be construed as the $S$ portion of the $S \pi O$ formula.

The ectoderm is critically involved producing the body's information and control systems, including the brain and articulatory organs of the mouth, tongue, lips, vocal folds, pharynx, and probably the larynx as well $[119,120]$, and is the source from which the germ cells will migrate to the gonads to form gametes. Therefore, it is the repository of crucial information for control and development of the present individual as well as future generations and it is logically the dominant element in the three distinct germ layers of the developing embryo.

\subsubsection{The Mesoderm}

The mesoderm, or middle layer of the embryonic disk, will develop into the skeleton, the bones and its marrow, as well as striated and smooth muscles. It provides the pluripotent stem cells that will form the heart and circulatory systems, the mobile parts of the digestive/excretory systems as well as the urinary systems and the kidneys, the lymphatic systems, and the sweat glands [118,119]. These elements are the main transport and mobility systems of the body corresponding to the $\pi$ portion of the $S \pi O$ formula.

They include both the mobility systems that come under the control of conscious intentions and volitions as well as the autonomous movements of the body. The latter are especially involved in the acquisition of critical supplies such as oxygen, water, and nutrients, as well as their transport to all of the organ systems throughout the body, and the exportation of waste products, toxins, and harmful disease agents through exhalation of carbon dioxide and the excretion of liquid wastes in sweat, urine, and feces. The heart is of particular interest as its movements are certainly affected by voluntary movements of other striated muscles and yet this highly organized system of striated muscle tissues, unlike the striated muscle tissues that normally fall under voluntary control, is ordinarily under the control of the autonomous nervous system. The rate of the heart's pumping, of course, is normally indirectly controlled by demands for oxygen that can be increased or decreased owing to intentional (volitional) movements of the body.

The mesoderm is also, as well we might expect seeing that it is the source of the essential autonomous mobility systems of the body, the primary source of the body's immune systems. These systems are deeply integrated into the transport involved in the importation of needed provisions, their distribution to all the organs of the body, and the exportation of gaseous, liquid, and solid waste products. The mesoderm is not only involved in producing those organ systems involved in nourishing and maintaining the body with respect to needed provisions that must be imported and wastes that need to be exported, but also with respect to the production of the elaborate defense systems that are commonly, and somewhat incorrectly referred to with a singular umbrella phrase as "the immune system." The body's internal defenses, however, are actually constituted by a deeply layered and richly integrated system of systems. 


\subsubsection{The Endoderm}

The endoderm, or inner layer of the embryonic disk, will develop into the inner layers of bodily surfaces in the lungs, the digestive tube except for the mouth, pharynx, and probably the larynx. The endoderm will provide the cells for the inner part of the rectum and the inner linings of glands which connect to the gut, including the stomach, colon, liver, pancreas, urinary bladder, urethra, epithelial linings of the trachea, lungs, pharynx, thyroid, parathyroid, and the intestines. The endoderm provides for the generation of the inner linings of the inner cavities of the ears, the urinary bladder, the inner parts of urethra as well as the inner linings of the thyroid gland and thymus [119]. Nearly all of the endocrine glands originate in this layer with the exception of part of the pituitary which arises in the ectoderm. When the work of the endoderm is completed, however, the envelope of the bodily icon with its many "surfaces within surfaces"- to use a phrase from Hoffmeyer [24] is well defined and fitted together as a whole. It seems strange to realize that the internal surfaces of the body are much larger and, with respect to the internal functions of the whole body, just as critical to its viability as its outer surface. For instance, the inner surface of the intestinal lining of an adult, if flattened out would, according to plausible estimates, cover an area about the size of a doubles tennis court [121].

The outer surfaces of the body, as already noted, are produced by the pluripotent cells of the ectoderm while all the internal organs not already provided for by the ectoderm, it seems, are generated by the mesoderm, but the innermost organ surfaces, those of the gut and of the organs that empty into it, or into the urinary tract, together with nearly all of the endocrine control and maintenance systems come from the endoderm. Thus, the endoderm effectively completes the boundaries of the bodily icon, the $O$, and the pragmatic mapping formula, $S \pi O$, is completed as the developing embryo comes to term and is readied for birth.

\subsection{Fetal Immune Defenses, Nutrition, and Clean-Up}

During the development of the viable fetus, the newly formed individual is provided oxygen and nutrients from mother's blood through a remarkable temporary circulation system that ingeniously prevents the mingling of the mother's blood with the baby's. The system not only protects the developing child from attack by infections, even ones that might affect the mother, but it also buffers the fetus from exposure to potentially harmful interactions that may take place in the mother's bloodstream and elsewhere while the baby is undergoing delicate and rapid development - the kind where a seemingly small problem could easily cascade into a series of harmful or even lethal effects downstream.

The red blood cells of the developing fetus are produced from the mesoderm of the blastocyst. However, the defense against infectious disease must be primarily provided by the mother's filtration and immune systems which clean her own bloodstream and secondarily by the only known type of the mother's immunoglobulin ( $\operatorname{IgG}$ ) that can pass across what is loosely known as the "placental barrier" - that barrier consisting of essential membranes that separate the mother's blood from that of the developing fetus: for one, there is the basal wall of the trophoblast bordering the intervillous space of the placenta that is richly penetrated by fetal capillaries; and, second, there is the basal wall of the fetal capillaries themselves. These membranes, and any space between them, must both be crossed in 
order for chemical materials - oxygen, carbon-dioxide, nutrients, and wastes - to pass between fetus and mother.

The whole placenta itself develops in the upper chamber of the blastocyst at the place where it is joined to the wall of the mother's uterus. It is a distinct organ generated by the germ cells of the blastocyst that is crucial to the development of the fetus and yet will be completely abandoned at the time of the newborn infant's separation from the mother with the severing of the umbilical cord. The placenta itself is a multilayered compartment contained within the mother's uterus. The outer layer of the placenta consists of a spongy material penetrated by maternal blood vessels, both veins and arteries, that supply maternal blood to the inside of an oddly shaped sac at the center of the placenta referred to as the intervillous space. The membrane forming the oddly shaped sac containing the intervillous space, sometimes merged with the outer basal membrane of the fetal capillaries, constitutes the essential "placental barrier." It is effectively wrapped around the branching tree like structures of blood vessels emanating from the developing fetus though the umbilical cord. The barrier membranes allow oxygen and nutrients to pass from mother's blood to the blood of the infant and at the same time to permit carbon dioxide and waste products from the fetal blood to pass to the mother's bloodstream.

The blood flow on the mother's side of the barrier is from the higher pressure arterial inputs toward the lower pressure venal tubes assuring an oxygen/nutrient rich supply of blood on the mother's side of the placental barrier that enables the fetal blood supply to expel its wastes while loading up with oxygen and nutrients for the return trip to the developing baby. Thus throughout the process the placenta does the work of the lungs, digestive, excretory, and immune systems of the developing baby.

The fetal circulatory system, though powered by the fetal heartbeat beginning at about the fifth to seventh week of gestation, is very different during fetal development than after birth. For one, the fetal red blood cells originating in the mesoderm have a higher affinity for oxygen than the ones that will be constructed after birth in the infant's bone marrow [122]. For another the flow of deoxygenated blood from the fetus back to the placenta occurs through the two higher pressure arteries flowing away from the fetal heart through the umbilical cord back to the placenta. This is unusual because, of course, arteries normally carry oxygenated blood.

Meanwhile oxygenated blood flows from the placenta to the fetus through a single large vein. Again, this flow contrasts with the later arrangement because in the fetus the large umbilical vein carries oxygenated blood toward the heart. This is almost exactly the opposite of the flow of oxygenated versus deoxygenated blood after birth when the umbilical cord will be cut and the neonate must breathe on its own. Another interesting peculiarity of the fetal circulation is that the oxygenated blood returning from the placenta to the fetus is divided at the liver with about half of it being filtered through the fetal liver while the other half goes on to the heart to be pumped through the developing fetal body, most of it bypassing the fetal lungs [123], and then returning after being deoxygenated in the fetal capillaries to the placenta through the dual umbilical arteries. The two umbilical arteries return deoxygenated blood and liquid wastes from the fetal bladder, to the placenta.

Although much of the blood flowing to the fetal liver bypasses it, the routing of roughly half the placental blood through the fetal liver suggests that some monitoring and filtering of materials in the oxygenated and nutrient laden blood is already taking place in the fetal liver. The developing liver also contains some blood stem cells that migrate to the thymus, where mobile lymphocytes, called T-cells 
(see section 4.9.4 below), mature and are tested and screened for autoimmunity. After birth, the formation of mature red blood cells and of the cells involved in the infant's immune systems will take place in the bone marrow.

\subsection{Neonatal Immune Defenses, Nutrition, Repair, and Clean-Up}

Presumably, during the rapid development of the blastocyst, so long as sufficiently capable totipotent or pluripotent stem cells survive an insult, damaged cells may be replaced or repaired on the fly. However, defense, repair, and clean-up capabilities internal to the individual become more critical after birth. For the first few months, especially if the baby is nursed with its mother's milk, it continues to benefit first from the residual IgG it received from its mother during gestation, and, subsequently, after birth it will continue to receive additional immune benefits through its mother's milk [124]. Of course, there is also the risk that even mother's milk may also be a source of toxins. For instance, contamination from heavy metals, e.g., from dental mercury, appears to be a risk during breast feeding, especially if the mother's exposure is substantial. For instance, the research shows plainly that placing or otherwise disturbing dental mercury (in dental amalgam) increases the exposure of the infant to this highly reactive toxin [125]. Also, it is known that combining risk factors, e.g., putting metal toxins together, may have a multiplicative effect rather than a summative one owing to the potential interactions. Likewise, combining toxins with disease agents provides for unpredictable opportunistic interactions [67].

High-risk combinations of toxins and disease agents may result from such diverse environmental sources as contaminated food or water, industrial wastes, pesticides, food preservatives, and even common medical procedures involving prescription drugs, dentistry, and vaccination. Even with prescribed drugs, antibiotics, vaccines (containing attenuated disease agents, adjuvants, animal proteins, and other adventitious components), and implantations of any kind placed in the body, clinical trials examining all possible interactions are prohibitively expensive even when the number of variables is less than or equal to three. The cost of clinical trials to examine possible interactions for numbers of variables greater than three grows at such an astronomical rate as to render studies of them essentially nonexistent. The lethal cases that involve multiple injuries from such interactions are apt to be recorded as some form of unexplained death—such as sudden infant death syndrome [67,126], anaphylactic shock, or simply as some unexplained variant of multiple systems damage attributed to unknown causes.

In nonlethal instances, interactive injuries may result in little more than a temporary malaise while in other cases the effects may result in a cascade of chronic, life-long and life-shortening illnesses, e.g., the disease condition known as emphysema associated with smoking and related hazards, or mesothelioma associated with asbestos exposure, and skin cancers related to excessive exposure to ultraviolet radiation come to mind. Because of the fact that damaged tissues are more susceptible to disease than healthy tissues, it follows that disease agents also have a greater chance of infecting persons who have already been damaged by toxins, radiation, or other injurious agents than persons who are in the peak of health.

For all of the foregoing reasons, it is unsurprising to discover that the body's developing systems of waste disposal and detoxification, are closely related to its systems of defense against disease agents. 
Similarly, we should expect, presumably, to find also that the body's systems of tissue repair will be intimately coordinated with both waste/toxin disposal and defense against invasive diseases. Likewise, when viewed from the side of potential disease agents that can invade the body-viruses, fungi, bacteria, and parasites - again it is unsurprising to find that undesirable microbes and organisms such as the common gut parasites known as pinworms and hookworms (along with many others that are less common) tend to bring a lot of undesirable company with them, both on their insides and on their outsides. Disease agents and parasites also tend to be loaded with toxic components that may sometimes be more harmful than the carrier. For all of these reasons, it is to be expected that the provisional requirements of breathing in and out, as well as nutritional requirements for water and food, must be expected to be intrinsically and deeply related to the detox, defense, and repair systems.

Therefore, it makes little or no sense to consider the immune systems as if they were isolated from or independent from the ordinary supply and maintenance systems that require interactions and communication between the biochemistry of the body and that of the external ecoment - to borrow an apt term from Brier [15] — with all of its potential hazards for injuries and diseases. However, in this paper, I have committed to deal primarily with the communication systems, in particular with the role of TNR type representations, crucial to the functioning of the immune defenses.

\subsection{Communication: Who Am I?}

Initially, the identity of self cells is defined in terms of the major histocompatibility complex (MHC) - derived from the nuclear DNA. This set of identity papers - consisting at maturity of hundreds of millions of copies of this TNR identifying the self, in effect, like a complex medical record and passport - provides the proof of identity of essentially every cell in the body of the neonate. At the beginning, in ways not yet fully understood, the body's totipotent cells were authorized to form any and every part of the human body. Progress from the totipotent cells to the differentiation that has occurred by six months after birth has presumably proceeded in large measure by communications from cell to cell during and after many mitotic divisions that have systematically constrained the expression of the DNA. The fertilized egg divides progressively into the cells of the blastocyst, the gastrula with its various layers, and so forth, right on up to the time of birth, after which, in typical cases the individual progresses to maturity.

About six months after the baby is born, just as the so-called "passive" immunity inherited from mother, fades in its effectiveness, the baby's own immune defenses, which have been ramping up from before its birth, are already, as Beck and Habicht [127] have said, "fabulously complex" (p. 60). In all its aspects and at almost every conceivable step, these incredible defense systems critically depend on successful communications in order to attain the homeostatic balance that we loosely refer to as "health and well-being." In medical jargon the contrast is between thriving and that vague status that often precedes a descent into disease loosely (and negatively) described as "failure to thrive" [119].

Questions concerning the identity of cells — whether they are legitimately part of the developing body, the self, or whether they are potentially harmful antigens, such as a toxin, an invading virus, fungus, microbe, or part of some other foreign organism (e.g., a gut worm or other parasite) — must be asked and answered correctly in order for the immune cells to do their work well. Also, it is useful for immune cells to be able to determine whether the cells of the self - the body to be defended, 
maintained, and possibly repaired - are healthy or possibly infected. If the cell is damaged and in need of help the immune cells may inquire concerning the nature and seriousness of the damage, infection, or deficiency in order to either correct it or send for help. In cases where the cell cannot properly identify itself, and/or report its status, the immune cell may be authorized either to kill and destroy it, or to call for help to accomplish the same purposes.

The cells that constitute the personnel for "immune" defense systems undergo processes that resemble production, nurturing and growth, program recruitment, training, hiring/firing, testing (sometimes of a do or die type), commissioning/decommissioning, arming/licensing, dispatching/ recalling, reassignment in some cases, long-term inactive status subject to call-up in case of war, and eventual retirement and/or disassembly in what would appear to be analogous to radical organ donation of recyclable chemical parts. Comparisons of the body's immune systems at a functional level can be drawn with ordinary municipal transport, supply, maintenance, waste disposal and sanitation departments, as well as many of the normal functions associated with fire and police departments. On a grander scale, the immune systems can be compared to defense systems on a national level by comparisons with the various branches of the military including information gathering resources, immigration control, Coast Guard/Border Patrol, common foot soldiers, elite commando units, and various other highly mobile forces performing such functions as reconnaisance, arrest, transport, detention, interrogation, execution, and disposal.

\subsection{TNRs at the Basis}

In all instances, success in fending off attacks and/or repairing any injuries invariably depends in the final analysis on the most mundane sorts of TNRs. Fictions, errors, lies, and gradations of nonsense all ride on the coat-tails of ordinary TNRs. With respect to potential life-threatening attacks, the body's systems evidently react conservatively by removing the perceived threat rather than merely observing or ignoring it. For this reason, friendly fire is always a risk in immune responses to infections and their accompanying toxic injuries.

Immune cells that are not reading reports correctly can begin to attack and destroy harmless antigens, as apparently happens in the case of allergies, or they can mistakenly attack the body's own healthy cells, tissues, and organs resulting in so-called "auto-immune" diseases [128], such as rheumatoid arthritis, multiple sclerosis, lupus, type 1 diabetes, and so on [129]. Other well-documented examples of what appear to be cases of autoimmunity can be found in the brain inflammation associated with autism and the general neuroinflammation found in multiple sclerosis and neuroAIDS, along with many other disorders [67]. All these appear to be produced in part by toxic/disease disruptions in the body's immune systems that end up by inadvertently (and somewhat desperately it seems) mistakenly attacking its own tissues and organs. In such extreme cases, the military seems to go completely berserk and take over the whole country - as manifested in full-blown life-threatening seizures commonly ending in death. In worst case scenarios, the whole nation collapses - the body dies. Life-threatening allergies and serious self-immunities fit these unpleasant descriptions.

In all such cases, where the immune functions get out of balance, defense resources appear to be misapplied because of mistaking false representations for true ones. In allergies as in all autoimmune 
diseases, it is evidently the case that the hint of a threat, though false, is taken to be real such that the perceived antigen is attacked and perhaps removed or destroyed, but not without collateral damage in the form of disruption of normal functions of bodily tissues and organs. In autoimmune diseases the collateral damage is greater in view of the fact that in those instances the immune cells begin to mistakenly treat the body's own healthy cells as genuine pathogenic antigens - as if the body's own cells were themselves disease agents. To prevent the cumulative damage, potentially catastrophic, of such mistakes, valid interpretations of TNRs are essential.

\subsection{A Multilayered System of Interacting Systems}

To truly appreciate the crucial importance of the balance that is ordinarily maintained by the normal interactions between the multiple layers of immune systems in a healthy human being, it is instructive to consider some of the ways that things can go radically wrong whenever one or more components of the multilayered system are thrown out of balance.

There are documented instances where this can be done unintentionally by a deliberate medical intervention. For instance, when penicillin has been administered in an effort to minimize the impact of an E. coli $\mathrm{O} 157$ infection, for instance, the fatality rate increases from approximately $15 \%$ to $50 \%$ of the persons infected [130]. The tripling of the potential lethality of the disease threat is attributable to the collateral damage to the ecoment of the gut by the administration of the antibiotic. To understand how such harmful effects - potentially deadly ones - can and do occur, it is necessary to take account of the interactions of the multilayered immune systems.

According to widely held and taught views of immunology three distinct levels of immune systems have been recognized. These include: (1) what is somewhat misleadingly called the "innate immune system," (2) the "complement system," and (3) the so-called "adaptive immune system" [131]. The inter-relatedness and cross-talk between these systems is increasingly evident in the current research and it is now widely agreed that a fourth layer of the vast immune network must be recognized in the gut microbial ecosystem. The ecoment of the gut, formerly a "forgotten organ" [132]—as O'Hara and Shanahan described it - is now increasingly thought of as an integral part of a normal and healthy body, perhaps even the "seat of the soul" as Andrew Wakefield has argued [133].

As can easily be seen from the current research, all four of the well-recognized (if not well-defined) layers of the system of immune systems are in fact each multilayered in its own. They are also deeply interrelated and exquisitely complex from top to bottom. The known functions of the immune system defy any piecemeal explanation. In a healthy body, the many layers of the systems work together with such efficiency that it seems necessary to suppose that almost any single component presupposes the whole. Here I want to suggest the hypothesis that the immune system of systems as a whole must be not only provided for within the initial DNA of the organism, but that its epigenetic and probabilistic expression must be implicit in systems provided for there as well. This is certainly not to deny that countless slips can occur between the DNA and its expression in bodily systems, but I do intend to suggest that there are compelling theoretical reasons to suppose that Crick's central dogma about the dominant role of DNA over RNA and both of them over proteins is probably more valid than many contemporary theoreticians might now suppose, e.g., Gottlieb, Stotz, and others [96,103]. 
There are also empirical evidences suggesting that the role of retroviruses and other potential means by which DNA can be modified on the fly tend to apply almost exclusively to the DNA of somatic cells rather than to the better protected and less accessible DNA of gamete cells. Generally epigenetic factors that can be shown to impact germ cell DNA do so in ways that either have unknown consequences, or, where the effects can be seen in the short term, they are disorder-producing, sometimes teratogenic, or lethal.

With all of the foregoing in mind, we will consider each of the foregoing systems one by one with special attention to their dependence on TNR type representations. Because each of the systems seems to presuppose the others, the task of introducing them is already difficult, but it is made even moreso by the fact that much of what has been claimed, and still is regarded by some as received wisdom, has already been shown to be false on the basis of empirical findings. Interestingly, the entire complex of inter-related immune systems begins from the premise that the self is to be protected from potential invasion or contamination by anything that is not either the self or identifiable as belonging in the neighborhood. This foundational premise of immune systems in general presupposes that every living organism with immune capacities, especially mammals and human beings, has a certain self-consciousness built into its cells right down to the level of its DNA.

It is intriguing to consider the possibility that self-consciousness, and perhaps consciousness its very self, consists of a certain resonance between the self cells of an entire body with all of its own organ systems. They seem to be tuned to a particular biochemical frequency shared by all those cells and organs that constitute the self. Theoreticians have variously tried to associate the "soul" and its consciousness - that ineffable whatever-it-is-ness that we call the "self" in modern talk - alternately with the whole body when it is awake, or especially with the brain or heart, or even with the gut, or possibly with the "personality" and its history, and so forth. However, what I want to suggest at this point is the possibility that the unifying character of consciousness, what is termed the "self"-as contrasted with "non-self"- resides within the very heart of our cells right down at the level of our initial DNA where the biologically viable and unique individual story of each of our respective selves begins.

For every mature human being capable of abstract thought, at some point there is a strange realization of self-awareness, self-consciousness, where the mundane ordinary sense of looking out toward an external world from within our body morphs into a representation of the fact that although we are in this body we cannot imagine how we got inside it, and yet, strangely, we can imagine what it would be like to be outside it as an observer. In fact, we find ourselves in such a relation to essentially all other human beings like ourselves. With such realizations come the great quandaries of existence. How did we get here? Where in the universe is the earth, anyway? And, how did the cosmos itself come to be? More significantly, how comes it that we are able to put these questions? In such a context, the question of what consciousness itself is seems to be intrinsically a problem of representation and of our capacity to produce at least some mundane true representations of the universe - apropos of the miracle referred to by Einstein [73] and of the resonance referred to by Peirce [72] and the profound quandary defined by Brier [14].

From the cellular point of view, the major histocompatibility complex (MHC) which, because of its crucial role in defining self to the body's immune systems, is also referred to as the human leukocyte antigen (HLA), actually involves several distinct levels of MHC/HLA. Only the red blood cells and 
the body's noncellular components do not rely on MHC to identify and define their roles to themselves and to other cells. The MHC inside each cell is the gene-richest part of the DNA. In higher vertebrates including humans, the MHC enables the production of certain proteins that are displayed on the surface of cells as identifiers of self and as a kind of written record of the history and current status of the cell.

In addition to its self identifier which marks it as a legitimate part of the body, the cell may also display non-self proteins or foreign proteins from invading microbiota (viruses and bacteria) that show it to be diseased or injured. Because these proteins are examined by specialized immune cells which may then trigger the production of antibodies that can identify, capture, and eventually destroy foreign microbiota that are the source of foreign proteins, the proteins displayed on the surface of cells are referred to as antigens (antibody generators). If the cell is damaged beyond repair, the immune inquirer may also call for it to be dismantled and removed from the body.

Abstracting and generalizing across the many complex layers of the immune systems, the key to success in protecting and maintaining the health of the self, is for the cells of the immune systems to correctly identify the body's own MHC proteins as self and to correctly recognize foreign, damaged, or non-self proteins as indicators of problems that need to be dealt with. If an MHC self-protein is validly reported by the cell to an immune cell inquirer, and if it is correctly taken as such by the immune cell, the result is a mundane exchange of valid TNRs between the cells. The construction of representations, however, is never completely without risk. As Peirce noted, chance is always a factor in present tense actions - and as Brier insists, we must acknowledge "chance as real" [14,15,134]. There is genuine risk in mistaking a mere fiction for an established TNR. This gives rise to the possibility of error and of deliberate deception as succinctly pointed out by Einstein in 1941 [135]. If misrepresentations accumulate sufficiently, as it seems they must by chance, they will eventually lead to dissolution and the death of the organism.

In individual cells the dissolution can come about gradually and naturally over the course of time resulting in what is known as apoptosis or it can come about in the context of mortal combat in conflicts of various sorts. In all instances, the success or failure of any given representation, all of which are more or less at risk in the present tense, depends ultimately on its truth or falsehood in the most mundane sense. In any individual body, truth is defined as agreement with the representations provided for by the organism's defining DNA, with special focus on the MHC.

\subsubsection{The Zero-Order Layer of Defenses: The Ecoment of the Gut}

According to standard doctrine - e.g., Alberts, et al., 1994 [136] - the most basic level of the immune defense systems consists of a whole complex of interrelated systems that are called "innate" to distinguish them from those that appear to be "acquired" or "learned" and that are accordingly referred to as the "adaptive immune system." However, it is somewhat misleading for a couple of reasons, to suppose that the so-called "innate" and "adaptive" systems are distinct in the manner the terms suggest.

First, it is evidently incorrect to suppose that all the various layers of the human immune systems are not provided for innately. The DNA-including its unfolding epigenetic expression and downstream consequences, however probabilistic and at risk they may be-nonetheless provides for 
that part of immunity which is referred to as "adaptive" just as it provides for the part that is referred to as "innate." The whole of the multilayered system of immune systems is undoubtedly provided for at least in an abstract way, though not yet in concrete detail, with the successful initial union of sperm and egg.

Second, the presumption that what is commonly referred to as "the innate immune system" is the most basic level is apparently also incorrect. There is a still more basic level of defense that confers a large part of the normal human's immunity to infection that precedes the entry of anything into the inside of the body itself - that is, into the blood, lymph, or bodily tissues beneath the epithelial layers that mark both the outside and the inside of the bodily icon.

Obviously, the skin and mucous membranes of the bodily orifices for the eyes, ears, nose, mouth, anus, urethra, and in females the vagina, provide an active line of defense against potentially harmful foreign substances and potential disease agents. They also provide necessary entry points for sensations of sight, hearing, smell, touch, taste, and sexual intercourse, as well as needed air, water, and food, and exit points for liquid and solid wastes. What is not so obvious is that material outside the inner linings of the body, including the linings of the digestive tube - the entire gut - is generally regarded as if it were still "outside" the body. The contents of the gut, for instance, according to gastroenterologists enter the body, by their reckoning, only by crossing through the lining of the gut into the blood, lymph, or other bodily tissues. Similarly, it can be argued that the gases in the lungs are not fully inside the body until they pass through the mucosal membranes into the blood and internal tissues.

Setting to one side the logical impreciseness of any such claims - an impreciseness that is partly unavoidable because the edges must be joined smoothly and the surfaces are not completely impermeable - it is nonetheless true that the borders of the gut in particular, especially the small and large intestines where much of the biochemical work of digestion is performed, are closely guarded. Also, it is in the human gut that we find what might well be termed the zero-order immune system of a healthy body. Vighi, Marcucci, Sensi, Di Cara, and Frati [137] estimate that "almost 70\% of the entire immune system" (p. 3) is contained within the gut—also see H. Miller, et al. [138]. No doubt this is one of the reasons why the United States National Institutes of Health has committed \$115 million to study to microbes of the body and especially of the gut [139]. Within the gut, there are, it is estimated, about 100 trillion biological microbes [140].

The vast majority of these gut inhabitants - commonly referred to as "microflora" but better described by the more general term "microbiota" (both of which are in common use) - are not only not harmful but, on the contrary, help us to digest materials essential to the body and stand as a zero-order defense against unwelcome intruders. O'Hara and Shanahan [132] have argued that the gut microbial system "has a collective metabolic activity equal to a virtual organ within an organ" (p. 688). It is evidently the case that there is considerable "cross-talk" between the microbiota of the gut and the host organism [141]. Serino, et al. point out that the communications between the microbiota of the gut and the host are evidently of vital importance to the host:

Over the past few years, it has been established that, in obesity, type I diabetes and Crohn's disease - to cite but a few - the intestinal microflora play a pathophysiological role and can induce, transfer or prevent the outcome of such conditions. A few of the molecular vectors responsible for this regulatory role have been determined. Some are related to control of the immune, vascular, endocrine 
and nervous systems located in the intestines. However, more important is the fact that the intestinal microflora-to-host relationship is bidirectional [142], with evidence of an impact of the host genome on the intestinal microbiome (especially see p. 162).

That is, it appears that the genetic specifications of the host determine in large measure the shaping and composition of the microbiota of the gut. Taking into consideration the extent to which the integumentary system of the host's body (the $O$ of the $S \pi O$ formula) is united through the tubes in the body's orifices with the inner mucosal linings of the gut and with all the organ systems that have contact with the external world, perhaps the interactions of the host genome with the ecoment of the gut logically suggests a great deal more about the unplumbed depths of functions of the genome. It seems likely that the genome represents much more about the extended neighborhood, the whole ecoment, of the organism than has been previously realized - a possibility more in keeping with the logical requirements of ethology as suggested by Brier in 2008 [14].

Elsewhere, Brier [16] has referred to the "transmitters in the brain and the immune system" which he suggests involve "interactions" that may be "very important for the establishment of the autopoeitic system of the second order" (p. 64). With reference to the gut microbiota, Round and Mazmanian [143] tantalizingly go so far as to suggest that the "disturbances in the bacterial microbiota" that evidently "result in dysregulation of adaptive immune cells raises the possibility that the mammalian immune system, which seems to be designed to control micro-organisms, is in fact controlled by micro-organisms" (p. 313). Surely, they go too far in supposing that a collection of many disparate, often conflicting micro-organisms, could do more than the coordinated system of self-cells, organs, and living tissues in the body of any individual mammal, much less any self-conscious ordinary human being, but there is no doubt that there is considerable cross-talk between the microbiota in the gut of a human being and the immune systems of that same person [141,144].

Among the recently discovered peculiarities of the interactions between the immune systems and the microbiota of the gut is evidence of changing roles of high level immune cells known as $\mathrm{T}$ regulators (Treg cells). These cells are part of what is thought of as the highest layer of the four global layers of the whole immune system-namely, the "adaptive immune system" (see section 4.9.4 below) - and they are involved in communications that inform and shape the development of the immune systems of infants as they mature toward adulthood. The research of Grindebacke, et al. suggests that the infant's Treg cells first focus attention on antigens that are appearing in the gut but increasingly attend to ones that originate outside the gut as the infant matures beyond its first 18 months of life [110]. What is interesting about the current research into such regulatory communications is that they show the various layers within the immune systems to be integrated in ways that are only just beginning to come to light. This will be even clearer as we proceed through the other global layers of the immune systems.

\subsubsection{The "Innate Immune System"}

Turning next to the somewhat misleadingly so-called "innate immune system" (ISS), we find again, a richly layered system of systems closely integrated with the other systems that theoretically, at least, reside above and below this major layer. Theoretically, the ISS ranks above the layer of immune functions of the gut microbiota and beneath the so-called "complement system" (CS) - the latter being 
in its turn is beneath the layer known as the "adaptive immune system" (AIS). However, because of the communications between the various hypothetically distinct layers, the boundaries between them are fuzzy to say the least.

The distinction between layers is more a matter of how far beneath the outer surfaces of the body the immune cells of the system in question penetrate. The microbiota of the gut, for instance, do not normally penetrate the lining of the gut to enter more deeply into the bloodstream or lymphatic ducts. However, the immune cells of the higher systems generally have access to the bloodstream and/or the lymphatic ducts - often referred to as the "humoral" immune system (with fast moving components that have access to all the major layers of the whole system of systems).

Another traditional basis for distinguishing the ISS and the AIS layers was the fact that only the latter layer can produce immunoglobulins (also known as antibodies) enabling defenses targeted against particular classes of disease agents. That distinction still stands. However, along that line it was commonly supposed that only AIS cells were able to acquire the information necessary to describe the identity of particular pathogen — as in a labeled wanted poster - in order to display it and thus communicate the nature of a particular attacker, an antigen, to other cells especially the attack cells of the immune systems. Immune cells that can display the identity of particular pathogens in this way are called antigen presenting cells (APCs). Until recently, it was supposed that only AIS cells could perform this function, but it is now known that all immune cells can probably serve as APCs.

All of this, however, goes to show that the foundational premise of the ISS - and, indeed the immune systems in general-is that there is a real difference between self cells and non-self cells. As already noted this distinction depends initially on the self defining major histocompatibility complex (MHC) which is also known as human leukocyte antigen (HLA). The most general type of this self-defining ID and health report is displayed by every cell in the body excepting those that lack a nucleus, e.g., red blood cells and blood platelets which are so generally necessary to the survival of every other self cell that to require identification from them would be like requiring yourself to show yourself your passport to verify your identity before every breath, every swallow of liquid, and every bite of food. It would be superfluous to the point of defeating the self nurturing functions of the red blood cells and platelets for them to display MHC to themselves and other self cells. It seems a little odd to call the self-defining MHC an "antigen" because it is - provided that it is a well-formed TNR faithfully representing the identity of the self-the foundational basis for defining non-self entities that can reasonably be referred to as "anti-gens" - or valid generators of antibodies. The self-defining MHC certainly is not normally intended to generate antibodies against itself or the cells and organs that it defines as self. That said, the self-defining MHC is commonly expressed on the outside of a cell. This common, self-MHC, then, mutually affirms itself to neighboring cells and to inquiring leukocytes somewhat in the manner of a license or passport photograph. All healthy self-cells, excepting red blood cells and thrombocytes for the reasons already noted, can identify themselves with MHC and ones that can also present information concerning the identity of pathogens are considered APCs. It has turned out that the APC function is much more general than formerly supposed.

Among the authorities checking for the identity of cells or standing guard against foreign biological matter inside the body are the immune cells of the ISS layer. With the exception of cells formed during fetal development and before the individual has developed bones, all the immune cells originate in the bone marrow from what are termed hematopoeitic stem cells. In addition to the basic workhorses of 
the bloodstream which consist first and foremost of the exceedingly numerous red blood cells - with about 200 billion being produced each day [145] — and secondarily of megakaryocytes that produce platelets and the various thrombocytes making up key components of blood plasma enabling blood clotting and the healing of injured tissues, the hematopoeitic stem cells of the bone marrow also produce various white blood cells-leukocytes of various types which are the main agents of the ISS, and, also, the AIS (discussed below in section 4.9.4). The hematopoietic stem cells that produce leukocytes, according to current understanding, are differentiated in the bone marrow into myeloid and lymphoid progenitors.

The leukocytes of the ISS can be differentiated into three main groups. First, there are five major kinds highly mobile ISS leukocytes, ones that move rapidly in the bloodstream and that can move out into the lymph and other tissues. Among the ISS mobile leukocytes are four that are believed to have a myeloid progenitor — neutrophils, eosinophils, basophils, and monocytes - and there is just one type, the natural killer (NK), that has a lymphoid progenitor. All of them are involved in dealing with perceived pathogens although the monocytes are also specialized for clean up and waste disposal. The NK cells are large lymphocytes critical for defense against cancerous cells. Normally they constitute about $10 \%$ to $15 \%$ of blood lymphocytes and were called "natural killers" because they were originally thought to be licensed to kill, like a kind of James Bond of the ISS, any cell not displaying self-identifying MHC. However, it has more recently been learned that NK cells are regulated by special "killer Ig[immunoglobulin]-like receptors" determining activation thresholds for these cells [146]. Again, contrary to prior thinking, NK cells though not yet shown to be fully functional APCs as the other ISS leukocytes evidently are (see discussion below), nevertheless, have turned out to be at least weak APCs [147].

Next, turning to the myeloid leukocytes, the most plentiful and most common type consists of the neutrophils whose main function is to consume and kill, by a process much like eating and digesting, invading bacteria or fungi. They do this by capturing one or several of the perceived foreign microbiota, engulfing them and then decomposing them through an internal digestive process that also results in the death of the leukocyte after only a few of the invaders have been dispatched. An accumulation of neutrophils is what appears as pus at the site of an infection. Neutrophils collectively account for about $50 \%$ to $70 \%$ of the total number of leukocytes circulating in a normal adult's blood at any given time. They are like police on patrol exercising lethal force against certain kinds of invaders that cannot identify themselves as "self" by displaying self-defining MHC.

In spite of the fact that traditional teaching in immunology suggests that neutrophils are generic attack units, it is evident that they sometimes attack certain kinds of invaders while ignoring others. In Figure 9, from Behnsen et al. [148], a neutrophil can be seen attacking and disposing of several Candida spores (the green spheres) while it ignores various Conidia (the white spheres). Does the neutrophil know the difference or is it constrained in a purely physical way as suggested by Behnsen et al.? It seems that neutrophils may be informed concerning what sort of bad guy to hunt down at the moment. The sort of representation required, in any case, for a deliberate search (hunt) is a hypostatic abstraction of a Candida spore that enables one to be recognized when found-perhaps as distinct from a Conidium. At that point the hypostatic icon of a Candida is matched with a perceived icon and the $S \pi O$ requirements are fulfilled - a TNR is achieved. It can easily be shown that more is required for a specific search, but certainly no less will work. Whether neutrophils are capable of such a search 
and destroy mission may be in doubt, but there is no doubt that adaptive immune cells can perform such missions.

In fact, the notion that neutrophils are naive generic immune cells was shown to be false by Cassatella, et al. in 1995 when they discovered that neutrophils themselves play a regulatory role in "adaptive" immunity [149]. This result has generally been confirmed and accepted [150,151]. Also, it has been shown that the premature apoptosis of large numbers of neutrophils is indicative of toxic stress [152] showing, again, the close coordination of detoxification and immune functions.

Figure 9. A neutrophil (the fast moving phagocytic leukocyte) can be seen attacking and disposing of several Canadian spores (the green spheres) while it ignores various Conidia (white spheres)-from Behrens, et al. (2007, Video S15).

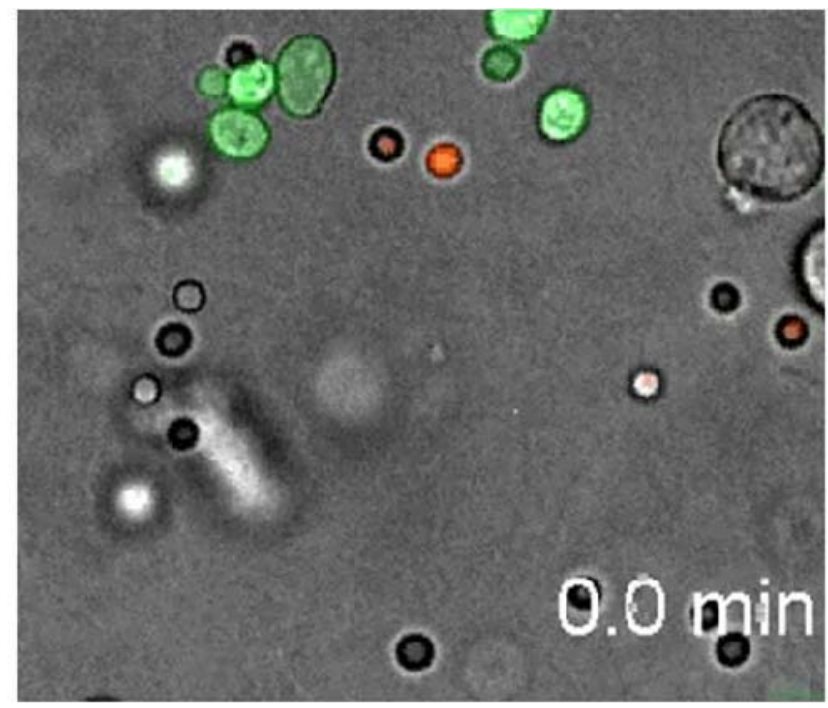

A less numerous type of myeloid mobile leukocytes, eosinophils, are particularly useful in attacking and warding off various parasites (e.g., helminths such as the common pinworms or hookworms often found in the intestines of mammals and human beings). Eosinophils account for about $1 \%$ to $6 \%$ of the total supply of leukocytes in a healthy adult bloodstream. They release toxic cytokines that are harmful to invading parasites but also to the host tissues. While they were formerly thought to be exclusively part of the traditionally described ISS it is now known that eosinophils also play an immunoregulatory role linking them both to the adaptive and innate layers of the immune systems [153-155].

A third type of myeloid mobile leukocyte, one traditionally associated with the ISS, is the basophil. It is a type accounting for only about .01 to .3 percent of leukocytes and as might be expected basophils also have a more specialized function. As granulocytes circulating in the blood they seem to be involved as signaling cells to notify other immune cells of an infection site or injury. They also are involved in healing and especially in producing cytokines involved in producing new blood vessels in damaged tissues [156]. Again, the implication arises that basophils are not only involved in the ISS but also in the AIS.

The three main mobile myeloid types of leukocytes (at the ISS level) seem to function as police with different specialties, and ranks, but they appear to be equipped to handle routine common problems rather than the sort of major attack from outside the body that would require a more highly 
trained professional military response (at the AIS level). The common neutrophils are like cops on patrol who can use lethal force in dealing with common domestic problems-bacteria and fungi. For bigger domestic problems, the eosinophils are like the SWAT team specialists on the police force that are called out to deal with difficult large problems - parasites such as the helminths that contain huge loads of bacteria, viruses, and toxins that need to be managed carefully and preferably encouraged to stay in their mobile homes and exit the body along with other wastes. The basophils are evidently a less common sort of detective specialized in gathering information, sounding the alarm when a problem occurs to call for assistance at the site of an injury or infection, and, in the meantime, to establish a perimeter and initiate damage control measures.

While all three types of mobile leukocytes are associated with the ISS - a layer just above the zeroorder rank of the gut microbiota - all of the mobile police in the ISS seem to be able to communicate with and to present problems about specific kinds of bad guys (antigens) to specialists in the better equipped and more highly trained professional military - the folks in the AIS. At any rate, neutrophils [157], eosinophils [153,154,155], and basophils as well [158], can function as antigen presenting cells (APCs) - that is, immune cells that report the existence of a particular perceived threat along with something like a labeled description of the suspects to be rounded up. Even when these mobile police on patrol are dying or already dead, any one of the three is capable of sending out an alarm to call for help. Bird points out that eosinophils and basophils can do so [159], and Yang, et al. [160] describe how it works with neutrophils:

alarmins released by neutrophil degranulation and/or death can link neutrophils to dendritic cells by promoting their recruitment and activation, resulting in the augmentation of innate and adaptive immune responses (p. 531).

The fourth class of myeloid mobile leukocytes consists of the highly versatile monocytes. Their specialty seems to be in cleaning up the scene of a crime-disposing of the bodies and debris left behind and putting things back in order. However, they too are capable of functioning as APCs [161] and can exercise lethal force. They are also able to move from the bloodstream into bodily tissues where they muscle up and specialize into either of two types of tissue resident outpost guard cells, macrophages or dendritic cells. Both are larger than the monocytes in the bloodstream. The macrophages still specialize in debris disposal and the clean up of tissues by phagocytosis. The other type of tissue resident cell that a monocyte may become is known as a dendritic cell because of its dendrite-like appendages that it uses to grab hold of foreign particles or antigens-e.g., see Figure 10 in which a dendritic cell can be observed to drag a conidium about $9 \mu \mathrm{m}$. All of these cells are capable of killing functions and of serving as APCs [157,162].

Interestingly, in 2008 Hume argued that the distinction between macrophages and dendritic cells with respect to their APC capabilities has been exaggerated. He insisted that the traditionally distinguished types should be collapsed into a single category and that macrophages are just as able to serve APC functions as dendritic cells [162]. With that idea in mind it seems reasonable to wonder if perhaps other current distinctions in levels and types of leukocytes and other immune cells may also need eventually to be reconsidered. For instance, are the basophil granulocytes in the bloodstream really distinct in origin and type from the category of leukocytes known as mastocytes (which are also called as "mast cells")? Whatever the case may be, the mast cells are very much like basophils in their 
form but like the so-called "fixed" location monocytes the mast cells also migrate into tissues where they specialize either as resident guards in mucosal membranes or as distinct types of specialized outposts in various other tissues [163,164]. Mast cells, it seems, have a lineage from the bone marrow distinct from that of the basophils which they greatly resemble. From the bloodstream, the mast cells migrate out to mucosal linings and bodily tissues where they take up residence as on location guards. Like basophils, they are able to regulate levels of inflammation and to mediate tissue repair [156].

Figure 10. A dendritic cell with extened appendages drags in a conidium, but does not ingest it-from Behrens, et al. (2007, Video S8).

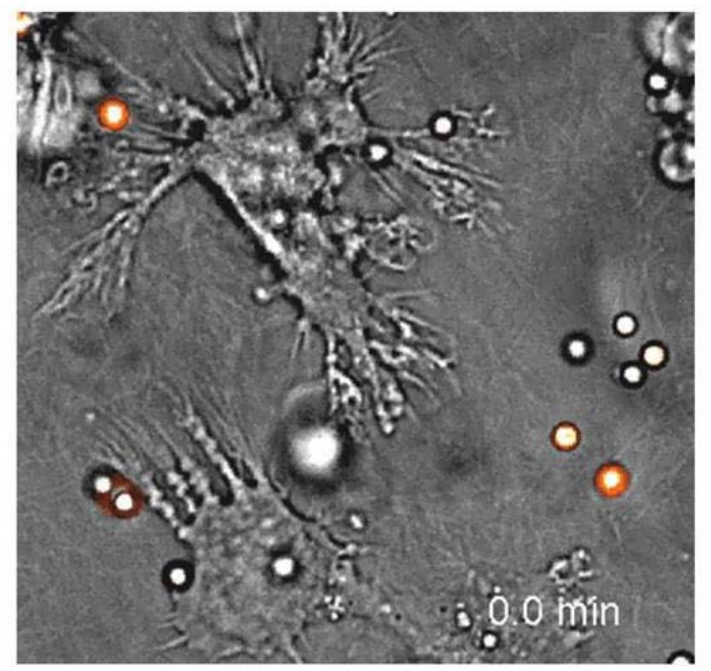

Summing up the functions of the ISS, they can be described as (1) the discovery of non-self agents and debris - a symbolic $(S)$ function; (2) the neutralization of non-self elements - an active indexical $(\pi)$ function; and (3) disposal of non-self agents and debris - an iconic $(O)$ function.

\subsubsection{The "Complement System"}

What is known as the "complement system" (CS) is a complex of several similar biochemical cascades enabling the mass destruction and disposal of non-self enemy combatants (pathogens) and/or the debris of non-self proteins. The basic components of the system are said to be produced by the ISS. The basic complement components consist of a substantial supply of proteins produced in the liver and circulating in the bloodstream that may be compared to a kind of complex weapon system consisting of multiple parts waiting for a signal to go active. The yet to be activated proteins circulating in the blood constitute something like a ready weapons cache awaiting a sequence of interlinked activating signals ultimately enabling the launching of ever so many tiny attack complexes that can be guided to pathogenic targets of opportunity where they form up on the surface of the marked target cell, perforate its outer surface and kill it. Before the complement protein system is activated, the targets, hopefully consisting of pathogenic non-self cells — rather than healthy self cells - are marked for destruction and removal in a variety of ways by the ISS or the AIS (see section 4.9.4 below).

The targeting of pathogens by the AIS involves their being marked by immunoglobulins (antibodies) IgG, IgM, or IgA bound to their surface. The marking by the ISS, by contrast, is less 
specific and involves some as yet to be determined means of chemical marking merely described as "pathogen-associated molecular patterns" (PAMPS) which supposedly enables CS targeting of common pathogens. Recent research by Paul-Clark, et al., however, suggest that oxidative damage may be sufficient in some cases at least to initiate CS targeting of cells as pathogens [165].

When the CS works well, the elements (antigens and wastes) to be disposed of are correctly identified and marked for destruction and removal after which the CS layer is triggered by some threshold of cytokine activity released by inflamed tissues, ISS, and AIS immune cells. When that happens, the biochemical cascade of the CS layer kicks into action and rapidly cleans house. It does so by bursting the cell membranes of identified pathogens (lysis), by enhancing the phagocytosis of perforated pathogens, and by speeding up the clearance of debris through the spleen and liver. In view of the fact that about $5 \%$ of the total blood serum may be recruited into the activity of the CS, the amplification of the capacity of the body to rid itself of disease producing pathogens and to clean up the debris of the collateral damage is enormous. The positive effects of the CS, when it works well - that is when the vast majority of targeted elements are pathogens and collateral damage to self cells is minimal - the impact may appear as something akin to overnight healing. The fever and symptoms may suddenly abate because of the impact of the CS response. On the other hand, because of the global impact of the CS on much of the body and on multiple organ systems, there is a concomitant risk of catastrophic results if things go wrong.

In worst case scenarios, the result can be anaphylactic shock followed by the sudden death of the organism. In less severe but still acute instances, violent seizures may result. From these examples it is easy to infer that combinations of difficulties and gradations of severity can fall anywhere on a scale from acute life-threatening multiple system failures to milder cases that may develop gradually to the point of being hardly detectible. By contrast, if the CS is working correctly, non-self elements are correctly identified, marked for destruction and removal, and subsequently are swept up in an extremely efficient way. The analogy that comes to mind is that of a killing hail storm followed by a tornado that sweeps through a war zone-the ballistic hail selectively targets and kills enemy combatants while leaving the defending immune cells and the civilian population virtually untouched. Right behind the hail of guided bullets comes a tornado-like vacuum sweeping up the debris of the war and the dead combatants.

\subsubsection{The "Adaptive Immune System"}

By now it is already clear that there are "adaptive" components throughout all of the foregoing immune system layers. Even the zero-order level of the ecoment of the gut microbiota involves cross-talk with higher levels suggesting that the gut microbiota also constitute, to some extent, an "adaptive" system. Also, as already pointed out, all of the cells of the ISS are capable of performing as APCs - a function formerly thought to be limited to the AIS.

Nevertheless, it remains true that the AIS is distinguished by its unique capacity to produce specific antibodies tuned to particular disease agents. In this respect, it stands above the microbiota of the gut, the ISS, and the CS layer. Together in communication with the other layers of the immune systems the lymphocyte cells of the AIS can target particular pathogens, track them down, immobilize, capture, 
and interrogate them to the point of dismantling them piece by piece, remember the results of prior engagements, and, thus, prepare in advance for possible repeat attacks.

From the lymphoid progenitors produced by the hematopoetic stem cells of the bone marrow, in addition to the large granular lymphocytes known as NK cells of the ISS which were discussed above, there are lymphoid dendritic cells [166]. These lymphoid cells, like myeloid dendritic cells, have the capacity to capture pathogens and deliver them to the lymph nodes. They both also have the capacity to phagocytose pathogens and cut their proteins into small pieces. Later, these fragments can be presented as antigen defining components on the surface of the dendritic cell. These cells function something like intelligence specialists teaching other cells about potentially harmful antigens - thus placing themselves among the so-called "professional" antigen-presenting cells-showing other cells in the AIS the differences between self-MHC (commonly referred to as Class I) and particular pathogen MHCs (Class II). There are evidently differences in the functions of lymphoid dendritic cells in contrast with their myeloid counterparts, but they are not yet well understood [144,167].

In addition to the lymphoid dendritic cells, the AIS also has small lymphocytes that originate in the bone marrow. These small lymphocytes are subsequently differentiated into B cells destined to develop antibodies against pathogenic non-self invaders and $\mathrm{T}$ cells that will go to the thymus for something like an advanced series of specialized military degrees in intelligence gathering and communication, command and control, and, some of the $\mathrm{T}$ cells will be specially equipped for hand-to-hand fighting against particular pathogens of which the T cells will be informed by APCs of the "professional" kind - those that enable the distinction between MHC-I, somewhat misleadingly called "self antigen," and MHC-II antigens that define perceived pathogens. It almost goes without saying that every step along the way, the representations that are provided to and by immune cells must conform to the requirements of TNRs, or disease, dissolution, and death of the organism will be the inevitable outcome.

The B cells, emanating from the bone marrow, must pass a series of do or die tests in before they can graduate to the level of B cells judged to be competent to produce antibodies. If the B cell happens to fail any of the tests along the way, it will either never mature, or it will dismantle itself by apoptosis. Upon its graduation as a B cell competent to produce antibodies, if it turns out that the antibody which it specifies happens to match up with the MHC-I that defines self-cells, that B cell will either become inactive or succumb to apoptosis. According to the current understanding, millions of different B cells with distinct antibodies are produced to circulate in the blood seeking a pathogen matching the description of the one that the particular B cell is able to produce and already has on hand. When a match is found, the B cell is authorized to engulf the enemy combatant, interrogate it to the point of digesting it, and then display the enemy's identity on its own (the B cell's) surface.

At this point the B cell is something like a well equipped military industrialist in a fast moving vehicle (a B cell in the bloodstream or lymphatic system) who has captured a prisoner and has extracted potentially vital information about an invasion possibly underway. The industrialist is able and willing to immediately set up one or more factories to produce the weapons (antibodies) that will enable the capture and destruction of the pathogen that has been identified, but needs confirmation that the information obtained is valid and the authorization to begin mass production. If and when the validation comes, the B cell will first clone itself multiple times, and then, its clones will convert themselves into plasma cells each of which engages in the manufacture of the needed antibody 
weapons. The confirmation and authorization to initiate the cloning of B cells and the production of plasma cell factories will come from T cells. A small fraction of the B cell clones will be transformed into long-lived B memory cells that will remain in the bloodstream conducting surveillance in case of a re-emergence of the same antigen at any time in the future. Some of these memory cells are known to last for the entire life of the host organism.

Consider next the $\mathrm{T}$ cells that are sent to the thymus for their training and education. It seems that the major classes of these specialists, all of which are in the military intelligence business, are critically dependent on gathering and confirming information from APCs. In the do or die training that these cells must undergo in the thymus, only about $2 \%$ of those that start the course will complete it. The rest will generally undergo inactivation and/or apoptosis. Initially, some of the graduating $\mathrm{T}$ cells will be immediately equipped with lethal cytotoxic capability involving a particular glycoprotein cluster of differentiation (CD8) that appears on its surface and binds to the MHC-I molecule [168]. The same glycoprotein is also found in NK cells within the ISS. Here, in the AIS, as soon as an APC informs the cytotoxic T-CD8+ cell about the precise antigen — one defined in an MHC-II molecule-it is ready to be authorized to go weapons hot and start hunting for the determined pathogen.

Meantime, another class of $\mathrm{T}$ cell, known in the immunology literature as a $\mathrm{T}$ helper (Th) cell - which is a specialist in intelligence gathering and communication with other immune system cells - will also be informed by a professional APC concerning an antigen that has been identified by that APC as an immediate threat. The informed Th cell —at this stage at Th0 cell, then — though it will not engage in direct combat with pathogenic cells, will proceed first to authorize the rapid deployment of informed killer $\mathrm{T}$ cells looking for the particular antigen of which the Th0 cell has been informed by the professional APC. The Th0 cell may next follow one of three branching routes: it may be biochemically transformed into a Th1 cell that will inform and encourage macrophages to attack the particular antigen the Th1 cell learned of from the professional APC; or the Th1 cell may be biochemically transformed into a memory $\mathrm{T}$ cell that will guard against possible future attacks by the same antigen.

Alternatively, the Th0 cell may be biochemically transformed into a Th2 cell that proceeds to authorize $\mathrm{B}$ cells to clone themselves and thus to initiate mass production of antibodies against the particular antigen the Th2 cell is able to confirm as an actual threat. After B cells clone themselves and produce plasma cells that manufacture antibodies against a particular antigen, the progress of the ongoing conflict will be monitored and regulated by interactions between B cells and Th1 cells. The result of the early Th0 activity in informing and authorizing killer T cells and macrophages to attack a particular pathogen will be to enhance the probability that the informed and active killer $\mathrm{T}$ cells and macrophages-provided that the MHC-II report is a TNR and that the killer $\mathrm{T}$ can correctly distinguish the MHC-I that defines the self cells via TNRs reported to the killer T from self-cellsmay be able to stop the pathogen before the infective agent has the chance to proliferate.

Finally, a few observations must be made about the remarkably simple and yet effective and vast differentiation of types of distinct immunoglobulins (particular antibodies). It is also important to include the caveat that in doing so, we have barely penetrated the inner workings of the four global layers of the immense system of immune systems. It should be noted as well that it is estimated that the number of distinct antibodies that can be produced by the human AIS exceeds 10 billion 
[169,170] - an observation by Susumu Tonegawa leading to a Nobel Prize awarded to him in 1987 [171]. At that time, the press release concerning Tonegawa's findings effusively claimed:

We are accordingly well prepared for an encounter with any possible antigen. It is likely that normally only a minor part of the antibody variance will ever be put into usage [172].

In fact, in 2009, Glanville, et al. showed that the combinatory possibilities for just one of the immunoglobulin types - namely, IgM, which is the third most plentiful of accounting for about $10 \%$ of the total antibodies-exceed $3.5 \times 10^{10}$ [169]. There are, in addition to this particular type of antibody, four other types: IgG, the general and most common type and the only one believed to cross the placental barrier; IgD, which seems to be involved in decommissioning immune cells; IgA, found mainly in mucosal linings; and the relatively rare, $\mathrm{IgE}$, which is associated with immune responses that can occur far from the evident site where the antigen impinges.

Although it is commonly supposed that the immune systems evolved gradually and in independent bits and pieces - on the theory that their known components seem to operate independently to a high degree - there are in fact, many indications that the systems are in communication with each other. The processes of manufacturing distinct types of cells in the bone marrow, their subsequent differentiation through various filters and developmental tests, along with the intricate systems of communication suggest that communications are occurring from the ecoment in the gut all the way to the highest levels of the AIS. Some of the means by which this communication occurs are understood, but it is plausible to suppose that a great deal more remains to be discovered.

\section{Summary and Conclusions}

This paper has summarized in an introductory way certain implications from a multi-layered general theory of signs stemming from Peircean semiotics. The general theory shows that all meaningful signs are dependent at their basis (in their origins) on ordinary true narrative representations (TNRs). A limit of absolute entropy, can be defined as the antithesis of the relatively well-grounded meaning discovered in TNRs. By contrast with the relatively well-determined, connected, and generalizable meanings in TNRs, a theoretical limit of complete entropy can be defined as the sort of indeterminacy, disconnectedness, and ungeneralizability of nothing at all or of everything with which a sign might conceivably be associated.

Well-formed TNRs - or at least their formal equivalents - it seems, are logical prerequisites to meaningful sign systems in general, and, therefore, to the formation of the genetic representations that provide the basis for living organisms. Incomplete fictional representations, for instance of an imagined artifact such as a microprocessor that has not yet been designed and manufactured - along the lines of Dasgupta's 1994 discourse on scientific creativity [22] — can sometimes be transformed from into TNRs by the production of the imagined artifact(s). Likewise, errors and even deliberate lies can sometimes be transformed into TNRs by discovering and replacing corrupted elements. For nonsense there is not much hope, which is why chaotic arrangements of matter make a difficult starting point for the accidental building up of the surpassingly complex and deeply layered TNRs found in genetic, epigenetic, and biochemical systems right on up to the highest levels of human neuroarchitecture and self-conscious experience. 
I have shown in this paper that the basic components of the simplest of TNRs can be found in the architecture of the human brain and in the manner in which it incorporates distinct icons of the body. With respect to genetic, epigenetic, and biochemical manifestations, again, it appears that the essential formal (logical) properties of TNRs are essential to biosemiotics across the board. While the details of the hierarchical relationship of the genome to bodily functions and to the ecoment of any organism, not to mention the intelligent living self-conscious human beings who are seeking to understand such representational systems, it appears from what we already know that valid (true) readings of representations from DNA through RNA to proteins are critical to normal functions at from genetics upward. Here, as noted throughout, the notion of "truth" is defined in its most mundane and least philosophical sense-i.e., as a balanced correspondence relation where the more abstract (or at least prior) element in the representation is dynamically fitted to its more concrete (or at least subsequent) content.

It was noted that the general sign theory discussed in this paper shows unequivocally-in a manner consistent with what Crick called the "central dogma" of molecular biology - that the flow of bio-cybernetic control is from the dominant symbol toward its subordinated object through an at risk active mapping relation. The general theory of signs discussed in this treatise requires the inference that the more abstract (or prior) element in every TNR - its $S$ as contrasted with its $O$ with which it must be associated via some dynamic $\pi$ mapping - holds the logical position of dominance and control (i.e., is the determiner of meaning). Logically, this is more certain than the empirical fact that the dominant hemisphere of the human brain tends to be the one that manages linguistic motor productions. The dominance relation of abstract symbols to their more concrete objects is also evident, however, as we have seen in this paper, in embryology and in the autopoeitic processes of somatic development in general.

The implications of all the foregoing for the development and functioning of human immune systems, insofar as they are coming to be understood, also entail their dependence on TNRs. The possibility of a fruitful development of hypotheses about immune functions along the lines of the theory of abstraction were left in the background, while it was shown that the foundational TNRs on which successful immune systems depend do not merely reside in the highly active nuclear DNA of the body's somatic cells, but are the essence of the self-defining element of the MHC/HLA that is commonly expressed on the surface of somatic cells. If those representations become corrupted or uninterpretable for any reason, autoimmunity of some sort seems inevitable. While we have probably just scratched the surface of the dynamic cross-talk that takes place within and between the various levels of the immune systems concerning identity, cell functionality, and no doubt a great deal more, such cross-talk is evidently occurring from the ecoment of the gut all the way up to the intelligence gathering and memory capacities of the adaptive immune systems.

Such empirical observations serve to illustrate the logical necessity of the claim that all meaningful signs - that is, ones that can enable some determination of content in a manner that connects with the universe of space, time, and matter and that is generalizable (whether by necessity or probability) across distinct portions of that apparent continuum - depend at their basis on those that meet the logical requirements of ordinary TNRs. Also the ubiquitous empirical presence of the foundational trinitarian sign triads — ones of the well-formed $S \pi O$ kind - throughout biosemiotic systems suggest that TNRs are more than merely abstract theoretical conceptions. When it was first suggested to me in 
the spring of 1983, by Jonathan de Berkeley-Wykes, then one of my PhD students at the University of New Mexico, that the foundational process of pragmatic mapping was, as he put it "hard-wired" into the neuroarchitecture of the human brain, I thought he was perhaps driving the theory too hard. In retrospect, however, I am reminded yet again of how easy it is to underestimate the value of a simple idea and thus to despise the day of small beginnings. Sadly, Jonathan died just eight years later, barely short of his 40th birthday, but his idea was closer to the truth than it was possible for me, at least, to realize at the time. He argued in its favor on the basis of his empirical work in clinical studies of bilingual aphasia, some aspects of which would appear later in a joint paper with Bruce Porch [173]. In conclusion, it now seems that the $S \pi O$ relation really does undergird the vesting of signs in general, including the biosemiotic systems, with whatever meaning they may have.

\section{References}

1. Augustine, St. Confessions of St. Augustine. In Great Books of the Western World; Hutchins, R.M., Ed.; Encyclopedia Britannica: Chicago, IL, USA, 1952; Volume 18.

2. Peirce, C.S. Truth. In Collected Papers of Charles Sanders Peirce; Hartshorne, C., Weiss, P., Eds.; Belknap Press: Cambridge, MA, USA, 1963; Volume 4; pp. 388-394.

3. Peirce, C.S. The Logic of Relatives. The Monist 1897, 7, 161-217.

4. Frege, G. Begriffsschrift, A Formula Language, Modeled Upon That of Arithmetic, for Pure Thought. In From Frege to Gödel: A Sourcebook in Mathematical Logic; van Heijenoort, J., Ed.; Harvard University Press: Cambridge, MA, USA, 1967; pp. 5-82.

5. Tarski, A. The Concept of Truth in Formalized Languages. In Logic, Semantics, and Metamathematics; Woodger, J. J., Ed.; Oxford University Press: Oxford, England, UK, 1956; pp. 152-278.

6. Tarski, A. The Semantic Conception of Truth. In Readings in Philosophical Analysis; Feigl, H., Sellars, W.; Eds.; Appleton: New York, NY, USA, 1949; pp. 341-374.

7. Oller, J.W. Reasons Why Some Methods Work. In Methods That Work: Ideas for Literacy $d$ Language Teachers; Heinle and Heinle: Boston, MA, USA, 1993; pp. 374-385.

8. Oller, J.W. How Grammatical Relations Are Determined. In The 22nd Linguistic Association of Canada and the United States (LACUS) Forum 1995; Hoffer, B., Ed.; Linguistic Association of Canada and the United States: Chapel Hill, NC, USA, 1996; pp. 37-88.

9. Oller, J.W.; Chen, L.; Oller, S.D.; Pan, N. Empirical Predictions from a General Theory of Signs. Discourse Process. 2005, 40, 115-144.

10. Oller, J.W.; Chen, L. Episodic Organization in Discourse and Valid Measurement in the Sciences. J. Quant. Linguist. 2007, 14, 127-144.

11. Peirce, C.S.A Guess at the Riddle. In Writings of Charles S. Peirce: A Chronological Edition 1886-1890; Houser, N.; Fisch, M.; Kloesel, C.J.W.; Moore, E.C.; Roberts, D.D.; Ziegler, L.A.; Atkinson, N.P., Eds.; Indiana University Press: Indianapolis, IN, USA, 2000; Volume 6.

12. Clark, R.W. Einstein: The Life and Times; H.N. Abrams: New York, NY, USA, 1984.

13. Luhmann, N. Essays on Self-Reference; Colombia University Press: New York, NY, USA, 1990.

14. Brier, S. Cybersemiotics: Why Information Is Not Enough; University of Toronto Press: Toronto, Canada, 2008. 
15. Brier, S. Information Seen as Part of the Development of Living Intelligence: The Five Leveled Cybersemiotic Framework for FIS. Entropy 2003, 5, 88-99. Available online: http://www.mdpi.org/entropy/papers/e5020088.pdf (accessed on March 20, 2010).

16. Brier, S. The Paradigm of Peircean Biosemiotics. Signs 2008, 2, $20-81$.

17. Shannon, C.E. A Mathematical Theory of Communication. Bell Sys. Tech. J. 1948, 27, 379-423; 623-656.

18. Wiener, N. Cybernetics or Control and Communication in the Animal and the Machine; MIT Press: Cambridge, MA, USA, 1961.

19. Wills, P.R. Informed Generation: Physical Origin and Biological Evolution of Genetic Codescript Interpreters. J. Theor. Biol. 2009, 2, 345-358.

20. Dawkins, R. Climbing Mount Improbable; Norton: New York, NY, USA, 1996.

21. Lakoff, G.; Johnson, M. Metaphors We Live By; Chicago University Press: Chicago, IL, USA, 1980.

22. Dasgupta, S. Creativity in Invention and Design: Computational and Cognitive Explorations of Technological Originality; Cambridge University Press: New York, NY, USA, 1994.

23. Koestler, A. The Act of Creation; Pan Books: London, UK, 1964.

24. A Legacy for Living Systems: Gregory Bateson as Precursor to Biosemiotics; Hoffmeyer, J., Ed. The Biocenter University of Copenhagen: Copenhagen, Denmark, 2008; Volume 2.

25. Oller, J.W. Adding Abstract to Formal and Content Schemata: Results of Recent Work in Peircean Semiotics. Appl. Linguist. 1995, 16, 273-306.

26. Oller, J.W.; Oller, S.D.; Badon, L.C. Milestones: Normal Speech and Language Development Across the Life Span; Plural Publishing: San Diego, CA, USA, 2006.

27. Titzer, R. J. Your Baby Can Read. Available online: http://www.infantlearning.com/robert.html (accessed on January 8, 2010).

28. Oller, J.W.; Giardetti, J.R. Images that Work: Creating Successful Messages in Marketing and High Stakes Communication; Quorum Books: Westport, CT, USA, 1999.

29. Oller, J.W. An Argument for Systems Grammar. Int. J. Ling. 2010, 2. Available online: http://www.macrothink.org/journal/index.php/ij1/ (accessed on March 20, 2010).

30. Peirce, C.S. Collected Papers of C. S. Peirce; Hartshorne, C., Weiss, P., Eds.; Belknap Press: Cambridge, MA, USA, 1931-1958; Volume 1-8.

31. Ohtsuka, K.; Brewer, W.F. Discourse Organization in the Comprehension of Temporal-order in Narrative Texts. Discl. Process. 1992, 15, 317-336.

32. Danesi, M. Explaining Change in Language: A Cybersemiotic Perspective. Entropy 2009, 11, 1055-1072.

33. Peirce, C.S. Collected Papers of C. S. Peirce; Hartshorne, C., Weiss, P., Eds.; Belknap Press: Cambridge, MA, USA, 1934; Volume 5.

34. Piaget, J. The Psychology of Intelligence; Routledge and Kegan Paul: London, UK, 1950.

35. Macknik, S.L. Visual Masking Approaches to Visual Awareness. Spanish J. Psychology 2007, 10, 478-479.

36. Martinez-Conde, S.; Macknik, S.L. Windows on the Mind. Sci. Am. 2007, 297, 56-63.

37. Bower, T.G.R. The Object in the World of the Infant. Sci. Am. 1971, 225, 30-38. 
38. Miller, G.A. The Magical Number 7, Plus or Minus 2-Some Limits on Our Capacity for Processing Information. Psych. Rev. 1956, 63, 81-97.

39. Ketner, K.L.; Putnam, H. The Consequences of Mathematics. In Reasoning and the Logic of Things; Ketner, K.L., Ed.; Harvard University Press: Cambridge, MA, USA, 1992; pp. 1-54.

40. Anderson, M.H.; Ensher, J.R.; Matthews, M.R.; Wieman, C.E.; Cornell, E. Observation of Bose-Einstein Condensation in a Dilute Atomic Vapor. Science 1995, 2, 198-201.

41. Tomasello, M. First Verbs; Cambridge University Press: London, UK, 1992.

42. Tomasello, M. Constructing a Language: A Usage-Based Theory of Language Acquisition; Harvard University Press: Cambridge, MA, USA, 2003.

43. Zipf, G.K. Human Behavior and the Principle of Least Effort; Addison-Wesley: Boston, MA, USA, 1949.

44. Grice, H.P. Logic and Conversation. In Syntax and Semantics, 3: Speech Acts; Cole, P., Morgan, J.L. Eds.; Academic Press: New York, NY, USA, 1975; pp. 41-58.

45. Bateson, G. Steps to an Ecology of Mind; University of Chicago Press: Chicago, IL, USA, 1973.

46. Ockham, W. Summa of Logic, Commentary on the First Book of the Sentences of Peter Lombard, and Explanations of Aristotle's "On Interpretation" (excerpts). In Ockham: Philosophical Writings; Boehner, P., Ed.; Bobbs-Merrill: Indianapolis, IN, USA, 1957; pp. 3546.

47. Oller, J.W.; Oller, S.D.; Badon, L.C. Cases: Introducing Communication Disorders Across the Life Span; Plural Publishing: San Diego, CA, USA, 2010.

48. Hagler, D.J.; Riecke, L.; Sereno, M.L. Parietal and Superior Frontal Visuospatial Maps Activated by Pointing and Saccades. Neuroimage 2007, 35, 1562-1577.

49. Jarrett, C., Barnes, G. The Use of Non-motion-based Cues to Pre-programme the Timing of Predictive Velocity Reversal in Human Smooth Pursuit. Exp. Brain Res. 2005, 164, 423-430.

50. Lichtheim, L. Ueber Aphasie. Aus der Medicinischen Klinik in Bern. Dtsch. Arch. Klin. Med., Leipzig 1885, 36, 204-268.

51. Sperry, R.W. Some Effects of Disconnecting the Cerebral Hemispheres. Available online: http://nobelprize.org/nobel_prizes/medicine/laureates/1981/sperry-lecture.html (accessed on Mar 19, 2010)

52. Gazzaniga, M.S. Cerebral Specialization and Interhemispheric Communication-Does the Corpus Callosum Enable the Human Condition? Brain 2000, 123, 1293-1326.

53. Biran, I.; Giovannetti, T.; Buxbaum, L.; Chatterjee, A. The Alien Hand Syndrome: What Makes the Alien Hand Alien? Cogn. Neuropsychol. 2006, 23(4), 563-582.

54. Loddenkemper, T.; Holland, K.D.; Stanford, L.D.; Kotagal, P.; Bingaman, W.; Wyllie, E. Developmental Outcome after Epilepsy Surgery in Infancy. Pediatrics 2007, 119, 930-935.

55. Merrick, E. Case study: Neuropsychologic Functioning and Complete Agenesis of the Corpus Callosum. Arch. Clin. Neuropsychol. 2009, 24, 474.

56. Paiement, P.; Champoux, F.; Bacon, B.A.; Lassonde, M.; Gagne, J.P.; Mensour, B.; Leroux, J.M.; Lepore, F. Functional Reorganization of the Human Auditory Pathways Following Hemispherectomy: An fMRI Demonstration. Neuropsychologia 2008, 46, 2936-2942. 
57. Paul, L.K.; Brown, W.S.; Adolphs, R.; Tyszka, J.M.; Richards, L.J.; Mukherjee, P.; Sherr, E.H. Agenesis of the corpus callosum: Genetic, Developmental and Functional Aspects of Connectivity. Nat. Rev. Neurosci. 2007, 8, 287-299.

58. Hofer, S.; Frahm, J. Topography of the Human Corpus Callosum Revisited-Comprehensive Fiber Tractography Using Diffusion Tensor Magnetic Resonance Imaging. Neuroimage 2006, 32, 989-994. Figure 1; Panel A.

59. Smith, A., \& Sugar, O. (1975). Development of Above Normal Language and Intelligence 21 Years after Left Hemispherectomy. Neurology 1975, 25, 813-818.

60. Gainotti, G. Different Patterns of Famous People Recognition Disorders in Patients with Right and Left Anterior Temporal Lesions: A Systematic Review. Neuropsychologia 2007, 45, 1591-1607.

61. Anaki, D.; Kaufman, Y.; Freedman, M.; Moscovitch, M. Associative (Prosop)Agnosia Without (Apparent) Perceptual Deficits: A Case-Study. Neuropsychologia 2007, 45, 1658-1671.

62. Esopenko, C.; Borowsky, R.; Cummine, J.; Sarty, G. Mapping the Semantic Homunculus: a Functional and Behavioural Analysis of Overt Semantic Generation. Brain Topogr. 2008, 21, 22-35.

63. Frazier, T.W.; Hardan, A.Y. A Meta-Analysis of the Corpus Callosum in Autism. Biol. Psychiatry 2009, 6, 935-941.

64. Keary, C.J.; Minshew, N. J.; Bansal, R.; Goradia, D.; Fedorov, S.; Keshavan, M.S.; Hardan, A.Y. Corpus Callosum Volume and Neurocognition in Autism. J. Autism Dev. Disord. 2009, 3, 834-841.

65. Penfield, W.; Boldrey, E. Somatic Motor and Sensory Representation in the Cerebral Cortex of Man as Studied by Electrical Stimulation. Brain 1937, 60, 389-443.

66. Purves, W.K.; Sadava, D.; Orians, G.H.; Heller, H.C. Life-The Science of Biology, 6th Edition; Sinauer Associates: Sunderland, MA, USA; 2001; p. 820.

67. Oller, J.W.; Oller, S.D. Autism: The Diagnosis, Treatment, and Etiology of the Undeniable Epidemic; Jones \& Bartlett: Sudbury, MA, USA, 2010.

68. Schell-Apacik, C.C.; Wagner, K.; Bihler, M.; Ertl-Wagner, B.; Heinrich, U.; Klopocki, E.; Kalscheuer, V.M.; Muenke, M.; von Voss, H. Agenesis and Dysgenesis of the Corpus Callosum: Clinical, Genetic, and Neuroimaging Findings in a Series of 41 Patients. Am. J. Med. Genet. 2008, 146A, 2501-2511.

69. Halassa, M.M.; Fellin, T.; Takano, H.; Dong, J.H.; Haydon, P.G. Synaptic Islands Defined by the Territory of a Single Astrocyte. J. Neurosci. 2007, 27, 6473-6477.

70. Mohler, H. Molecular Regulation of Cognitive Functions and Developmental Plasticity: Impact of GABA(a) Receptors. J. Neurochem. 2007, 102, 1-12.

71. Oller, J.W. Semiotic Theory Applied to Free Will, Relativity, and Determinacy: Or Why the Unified Field Theory Sought by Einstein Could Not Be Found. Semiotica 1996, 108, 199-244.

72. Peirce, C.S. A Neglected Argument for the Reality of God. Hibbert J. 1908 7, 90-112.

73. Einstein, A. Physics and Reality. In Out of My Later Years; Citadel: Secaucus, NJ, USA, 1936; pp 59-96.

74. Crick, F.H.C. On Protein Synthesis; Symp. Soc. Exp. Biol. 1958, 12, 139-163. 
75. Crick, F.H.C. Central Dogma of Molecular Biology. Nature 1970, 227, 561-563.

76. Pasteur, L. On Spontaneous Generation; Revue des Cours Scientifics, 1864, 1, 257-264.

77. Ludovic, M. Syntropy and Entropy in Self-Organized Systems. InterJ. Complex Sys. 2006, 223, 1-14.

78. Peirce, C.S. [On a Method of Searching for the Categories]: MS 133. In Writings of Charles $S$. Peirce: A Chronological Edition; Fisch, M., Kloesel, C.J.W., Moore, E.C., Roberts, D.D., Ziegler, L.A., Atkinson, N.P., Eds.; Indiana University Press, Peirce Edition Project: Indianapolis, IN, USA, 1982; Volume 1; pp. 515-528.

79. Writings of Charles S. Peirce: A Chronological Edition; Kloesel, C.J.W., Fisch, M., Roberts, D.D., Houser, N., Eds. Indiana University Press, Peirce Edition Project: Indianapolis, IN, USA; 1984; Volume 3.

80. Peirce, C.S. The Law of Mind. The Monist 1892, 2, 533-559.

81. Kharasch, M.S.; Mcbay, H.C.; Urry, W.H. Reactions of Atoms and Free Radicals. VII. Diacetyl Peroxide as an Agent for Linking $\alpha$-Carbon to $\alpha$-Carbon Atoms in Organic Esters. J. Org. Chem. 1945, 10, 394-400.

82. Westheimer, F.H. Morris Selig Kharasch 1895-1957: A Biographical Memoir; National Academy of Sciences: Washington, DC, USA; 1960. Available online: http://books.nap.edu/ html/biomems/mkharasch.pdf (accessed on March 20, 2010).

83. Netzer, N.; Goodenbour, J.M.; David, A.; Dittmar, K.A.; Jones, R.B.; Schneider, J.R.; Boone, D.; Eves, E.M.; Rosner, M.R.; Gibbs, J.S.; Embry, A.; Dolan, B.; Das, S.; Hickman, H.D.; Berglund, P.; Bennink, J.R.; Yewdell, J.W.; Pan, T. Innate Immune and Chemically Triggered Oxidative Stress Modifies Translational Fidelity. Nature 1990, 4, 522-526.

84. Luo, S.; Levine, R.L. Methionine in Proteins Defends Against Oxidative Stress. FASEB J. 2008, 23, 464-472.

85. Ibba, M.; Soll, D. Aminoacyl-tRNA Synthesis. Annu. Rev. Biochem. 2000, 69, 617-650.

86. Cochella, L.; Green, R. Fidelity in Protein Synthesis. Curr. Biol. 2005, 15, R536-R540.

87. Hoffman, G.W. On the Origin of the Genetic Code and the Stability of the Translation Apparatus. J. Mol. Biol. 1974, 86, 349-362.

88. von Uexküll, J.A Stroll through the Worlds of Animals and Men: A Picture Book of Invisible Worlds. In Instinctive Behavior: The Development of a Modern Concept; Schiller, C.H., Ed.; International Universities Press, Inc.: New York, NY, USA, 1957; pp 5-80.

89. Pellionisz, A.J. The Principle of Recursive Genome Function. Cerebellum 2009, 7, 348-359.

90. Jones, R.E.; Lopez, K.H. Human Reproductive Biology, 3rd Edition.; Elsevier: San Diego, CA, USA, 2006.

91. Saling, P.M. How the Egg Regulates Sperm Function During Gamete Interaction-Facts and Fantasies. Biol. Reprod. 1991, 44, 246-251.

92. Familiari, G.; Heyn, R.; Relucenti, M.; Sathananthan, H. Structural Changes of the Zona Pellucida During Fertilization and Embryo Development. Front. Biosci. 2008, 13, 6730-6751.

93. Wagman, J.B.; Miller, D.B. Nested Reciprocities: The Organism-Environment System in Perception-Action and Development. Dev. Psychobiol. 2003, 4, 317-334.

94. Klinkert, B.; Narberhaus, F. Microbial Thermosensors. Cell. Mol. Life Sci. 2009, 6, 2661-2676. 
95. Biro, J.C. Seven Fundamental, Unsolved Questions in Molecular Biology-Cooperative Storage and Bi-Directional Transfer of Biological Information by Nucleic Acids and Proteins: An Alternative to "Central Dogma". Med. Hypotheses 2004, 63, 951-962.

96. Gottlieb, G. Normally Occurring Environmental and Behavioral Influences on Gene Activity: From Central Dogma to Probabilistic Epigenesis. Psychol. Rev. 1998, 1, 792-802.

97. Temin, H. Homology between RNA from Rous Sarcoma Virus and DNA from Rous Sarcoma Virus-Infected Cells. Proc. Natl. Acad. Sci. U.S.A. 1964, 52, 323-329.

98. Prusiner, S.B. Novel Proteinaceous Infectious Particles Cause Scrapie. Science 1982, 216, 136-144.

99. Baltimore, D. RNA-Dependent DNA Polymerase in Virions of RNA Tumour Viruses. Nature 1970, 226, 1209-1211.

100. Baltimore, D. The Nobel Prize in Physiology or Medicine 1975. Available online: http://nobelprize.org/nobel_prizes/medicine/laureates/1975/baltimore-autobio.html (accessed on March 20, 2010).

101. Morange, M. The Central Dogma of Molecular Biology: A Retrospective after Fifty Years. $J$. Biosci. 2008, 33, 171-175.

102. Morange, M. The Central Dogma of Molecular Biology: A Retrospective after Fifty Years. Resonance 2009, 9, 236-247.

103. Stotz, K. Molecular epigenesis: Distributed Specificity as a Break in the Central Dogma. Hist. Philos. Life Sci. 2006, 2, 533-548.

104. Rosenberg, A. Is Epigenetic Inheritance a Counterexample to the Central Dogma? Hist. Philos. Life Sci. 2008, 2, 549-565.

105. Woese, C.R. Translation: In Retrospect and Prospect. RNA 2001, 7, 1055-1067.

106. Hoyle, F.; Wickramasinghe, N.C. The Theory of Cosmic Grains. Kluwer Academic: Dordrecht, Netherlands, 1991.

107. Forterre, P. The Origin of Viruses and Their Possible Roles in Major Evolutionary Transitions, Virus Research 2006, 117, 5-16.

108. Forterre, P.; Prangishvili, D. The Origin of Viruses. Res. Microbiol. 2009, 160, 466-472.

109. Weismann, A. On heredity. In Essays upon Heredity; Oxford: Clarendon Press, 1889; pp 67105. Available online: http://www.esp.org/books/weismann/essays/facsimile/ (accessed on January 5, 2010).

110. Grindebacke, H.; Stenstad, H.; Quiding-Jarbrink, M.; Waldenstrom, J.; Adlerberth, I.; Wold, A.E.; Rudin, A. Dynamic Development of Homing Receptor Expression and Memory Cell Differentiation of Infant CD4(+)CD25(High) Regulatory $\mathrm{T}$ Cells. J. Immunol. 2009, 1, 4360-4370.

111. Sutovsky, P.; Moreno, R.D.; Ramalho-Santos, J.; Dominko, T.; Simerly, C.; Schatten, G. Ubiquitin Tag for Sperm Mitochondria. Nature 1999, 402, 371-372.

112. Schwartz, M.; Vissing, J. New Patterns of Inheritance in Mitochondrial Disease. Biochem. Biophys. Res. Commun. 2003, 3, 247-251.

113. Fallarino, F.; Grohmann, U.; Vacca, C.; Orabona, C.; Spreca, A.; Fioretti, M.C.; Puccetti, P.T Cell Apoptosis by Kynurenines. Developments in Tryptophan and Serotonin Metabolism-Book Series: Advances in Experimental Medicine and Biology 2003, 20, 183-190. 
114. Bandelt, H.J.; Kong, Q.P.; Parson, W.; Salas, A. More Evidence for Non-Maternal Inheritance of Mitochondrial DNA?. J. Med. Genet. 2005, 4, 957-960.

115. Bandelt, H.J.; Yao, Y.G.; Bravi, C.M.; Salas, A.; Kivisild, T. Median Network Analysis of Defectively Sequenced Entire Mitochondrial Genomes from Early and Contemporary Disease Studies. J. Hum. Genet. 2009, 5, 174-181.

116. Mitalipov, S.; Wolf, D. Totipotency, Pluripotency and Nuclear Reprogramming. Engineer. Stem Cells Book Ser.: Adv. Biochem. Eng. Biotechnol. 2009, 114, 185-199.

117. Herrera-Gonzalez, N.E.; Dresser, D.W. Fetal-Maternal Immune Interaction: Blocking Antibody and Survival of the Fetus. Dev. Comp. Immunol. 1993, 1, 1-18.

118. National Center for Biotechnology Information (NCBI). What Is a Cell? Available online: http://www.ncbi.nlm.nih.gov/About/primer/genetics_cell.html (accessed on Jan 5, 2010).

119. Evers, C.A.; Starr, L. Biology: Concepts and Applications, 6th Edition; Thomson: Tampa, FL, USA, 2006.

120. Jonker, L.; Kist, R.; Aw, A.; Wappler, I.; Peters, H. Pax9 Is Required for Filiform Papilla Development and Suppresses Skin-Specific Differentiation of the Mammalian Tongue Epithelium. Mech. Dev. 2004, 1, 1313-1322.

121. Ward, J.P.T.; Clarke, R.; Clarke, R.W.; Linden, R.W.A. Physiology at a Glance. Blackwell Publishing: Boston, MA, USA; 2005.

122. Whitaker, K.B. Comprehensive Perinatal and Pediatric Respiratory Care; Delmar: Albany, NY, USA, 2001.

123. Moore, K.L.; Persaud, T.V.N. The Developing Human: Clinically Oriented Embryology, 8th ed; W.B. Saunders Company: Philadelphia, PA, USA, 2008.

124. Dorea, J.G. Health Hazard for Infants: Breast-Milk Mercury or Non-Breastfeeding? A Dilemma for Mothers Exposed to Mercury Vapors. Int. J. Hyg. Environ. Health. 2009, 2, 233-234.

125. Da Costa, S.L.; Malm, O.; Dorea, J.G. Breast-Milk Mercury Concentrations and Amalgam Surface in Mothers from Brasilia, Brazil. Biol. Trace Elem. Res. 2005, 106, 145-151.

126. Silvers, L.E.; Ellenberg, S.S.; Wise, R.P.; Varricchio, F.E.; Mootrey, G.T.; Salive, M.E. The Epidemiology of Fatalities Reported to the Vaccine Adverse Event Reporting System 19901997. Pharmacoepidemiol. Drug Saf. 2001, 1, 279-285.

127. Beck, G.; Habicht, G.S. Immunity and the Invertebrates. Sci. Am. 1996, November, 60-66.

128. Harrington, L.E.; Hatton, R.D.; Mangan, P.R.; Turner H.; Murphy, T.L.; Murphy, K.M.; Weaver, C.T. Interleukin 17-Producing CD4+ Effector T Cells Develop Via a Lineage Distinct from the T Helper Type 1 and 2 Lineages. Nat. Immunol. 2005, 6, 1023-1032.

129. Jepson, B.; Johnson, J. Changing the Course of Autism: A Scientific Approach for Parents and Physicians; Sentient: Boulder, CO, USA, 2007.

130. Boedeker, E.C.; Serna, A. Pathogenesis and Treatment of Shiga Toxin-Producing Escherichia Coli Infections. Curr. Opin. Gastroenterol. 2008, 24, 38-47.

131. Tosi, M.F. Innate Immune Responses to Infection. J. Allergy Clin. Immunol. 2005, 1, 241-249.

132. O'Hara, A.M.; Shanahan, F. The Gut Flora as a Forgotten Organ. EMBO Rep. 2006, 7, 688-693.

133. Wakefield, A.J. The Seat of the Soul: The Origins of the Autism Epidemic. Presentation at the Carnegie Mellon Institute, Pittsburgh, PA, USA, 17 November 2005. Available online: http://www.chem.cmu.edu/wakefield/ (accessed on March 20, 2010). 
134. Brier, S. Information and Consciousness: A Critique of the Mechanistic Concept of Information. Cybern. Hum. Know. 1992, 1, 71-94.

135. Einstein, Albert. The Common Language of Science. In out of My Later Years; Citadel: Secaucus, NJ, USA, 1956; pp. 111-113.

136. Alberts, B.; Bray, D.; Lewis, J. Raff; M. Roberts, K.; Watson, J.D. Molecular Biology of the Cell. Garland Publishing; Taylor \& Francis Group: London, UK, 1994.

137. Vighi, G.; Marcucci; F.; Sensi, L.; Di Cara; G.; Frati, F. Allergy and the Gastrointestinal System. Clin. Exp. Immunol. 2008, 153, 3-6.

138. Miller, H.; Zhang, J.; Kuolee, R.; Patel, G.B.; Chen, W. Intestinal M Cells: The Fallible Sentinels? World J. Gastroenterol. 2007, 13, 1477-1486.

139. Human Microbiome Project. Available online: http://nihroadmap.nih.gov/hmp/ (accessed on March 20, 2010).

140. Backhed, F.; Ley, R.E.; Sonnenburg, J.L.; Peterson, D.A.; Gordon, J.I. Host-Bacterial Mutualism in the Human Intestine. Science 2005, 3, 1915-1920.

141. Possemiers, S.; Grootaert, C.; Vermeiren, J.; Gross, G.; Marzorati, M.; Verstraete, W.; Van de Wiele, T. The Intestinal Environment in Health and Disease-Recent Insights on the Potential of Intestinal Bacteria to Influence Human Health. Curr. Pharm. Des. 2009, 1, 2051-2065.

142. Serino, M.; Luche, E.; Chabo, C.; Amar, J.; Burcelin, R. Intestinal Microflora and Metabolic Diseases. Diabetes Metab. 2009, 3, 262-272.

143. Round, J.L.; Mazmanian, S.K. The Gut Microbiota Shapes Intestinal Immune Responses During Health and Disease. Nat. Rev. Immunol. 2009, 9, 313-323.

144. Steinbrink, K.; Mahnke, K.; Grabbe, S.; Enk, A.H.; Jonuleit, H. Myeloid Dendritic Cell: From Sentinel of Immunity to Key Player of Peripheral Tolerance? Hum. Immunol. 2009, 7, 289-293.

145. Cecil Medicine. 23rd Edition; Goldman, L., Ausiello, D., Eds.; Saunders Elsevier; Philadelphia, PA, USA, 2007.

146. Purdy, A.K.; Campbell, K.S. Natural Killer Cells and Cancer Regulation by the Killer Cell Ig-Like Receptors (KIR). Cancer Biol. Ther. 2009, 8, 2211-2220.

147. Burt, B.M.; Plitas, G.; Nguyen, H.M.; Stableford, J.A.; Bamboat, Z.M.; DeMatteo, R.P. Circulating HLA-DR+ Natural Killer Cells Have Potent Lytic Ability and Weak Antigen-Presenting Cell Function. Hum. Immunol. 2008, 6, 469-474.

148. Behnsen, J.; Narang, P.; Hasenberg, M.; Gunzer, F.; Bilitewski, U. Environmental Dimen-sionality Controls the Interaction of Phagocytes with the Pathogenic Fungi Aspergillus fumigatus and Candida albicans. PLoS Pathog. 2007, 3. doi:10.1371/journal.ppat.0030013. Available online: http://www.plospathogens.org/article/info\%3Adoi\%2F10.1371\%2Fjournal.ppat.0030013 (accessed on March 20, 2010).

149. Cassatella, M.A.; Meda, L.; Gasperini, S.; Dandrea, A.; Ma, X.J.; Trinchieri, G. Interleukin-12 Production by Human Polymorphonuclear Leukocytes. Eur. J. Immunol. 1995, 2, 1-5.

150. Boudaly, S. Activation of Dendritic Cells by Polymorphonuclear Neutrophils. Front. Biosci. 2009, 1, 1589-1595.

151. Cassatella, M.A. On the Production of TNF-Related Apoptosis-Inducing Ligand (TRAIL/Apo2L) by Human Neutrophils. J. Leukoc. Biol. 2009, 7, 1140-1149. 
152. Sweet, L.I.; Passino-Reader, D.R.; Meier, P.G.; Omann, G.A. Effects of Polychlorinated Biphenyls, Hexachlorocyclohexanes, and Mercury on Human Neutrophil Apoptosis, Actin Cytoskelton, and Oxidative State. Environ. Toxicol. Pharmacol. 2006, 22, 179-188.

153. Akuthota, P.; Wang, H.B.; Spencer, L.A.; Weller, P.F. Immunoregulatory Roles of Eosinophils: A New Look at a Familiar Cell. Clin. Exp. Allergy 2008, 3, 1254-1263.

154. Blanchard, C.; Rothenberg, M.E. Biology of the Eosinophil. Adv. Immunol.-Book Ser. 2009, $101,81-121$.

155. Shi, H. Eosinophils Function as Antigen-Presenting Cells. J. Leukoc. Biol. 2004, 76, 520-527.

156. Crivellato, E.; Travan, L.; Ribatti, D. Mast Cells and Basophils: A Potential Link in Promoting Angiogenesis During Allergic Inflammation. Int. Arch. Allergy Immunol. 2010, 1, 89-97.

157. Ashtekar, A.R.; Saha, B. Poly's Plea: Membership to the Club of APCs. Trends Immunol. 2003, 24, 485-490.

158. Wynn, T.A. Basophils Trump Dendritic Cells as APCs for $\mathrm{T}(\mathrm{H}) 2$ Responses. Nat. Immunol. 2009, 10, 679-681.

159. Bird, L. Allergy and Asthma: Calling All Th2 Cells. Nat. Rev. Immunol. 2008, 8, 162.

160. Yang, D.; de la Rosa, G.; Tewary, P.; Oppenheim, J.J. Alarmins Link Neutrophils and Dendritic Cells. Trends Immunol. 2009, 30, 531-538.

161. Fricke, I.; Mitchell, D.; Petersen, F.; Bohle, A.; Bulfone-Paus, S.; Brandau, S. Platelet Factor 4 in Conjunction with IL-4 Directs Differentiation of Human Monocytes into Specialized AntigenPresenting Cells. FASEB J. 2004, 1, 1588-1597.

162. Hume, D.A. Macrophages as APC and the Dendritic Cell Myth. J. Immunol. 2008, 1, 58295835.

163. Prussin, C.; Metcalfe, D.D. IgE, Mast Cells, Basophils, and Eosinophils. J. Allergy Clin. Immunol. 2003, 112, 267.

164. Prussin, C.; Metcalfe, D.D. IgE, Mast Cells, Basophils, and Eosinophils. J. Allergy Clin. Immunol. 2006, 1, S450-S456.

165. Paul-Clark, M.J.; Sorrentino, R.; Bailey, L.K.; Sriskandan, S.; Mitchell, J.A. Gram-Positive and Gram-Negative Bacteria Synergize with Oxidants to Release CXCL8 from innate Immune Cells. Mol. Med. 2009, 1, 238-246.

166. Austyn, J.M. Lymphoid Dendritic Cells. Immunology 1987, 62, 161-170.

167. Darmochwal-Kolarz, D.; Rolinski, J.; Buczkowski, J.; Tabarkiewicz, J.; Leszczynska-Gorzelak, B.; Zych, I.; Oleszczuk, J. CD1c(+) Immature Myeloid Dendritic Cells Are Predominant in Cord Blood of Healthy Neonates. Immunol. Lett. 2004, 9, 71-74.

168. Leahy, D.J., Axel, R., Hendrickson, W.A. Crystal Structure of a Soluble Form of the Human T Cell Coreceptor CD8 at 2.6 A Resolution. Cell 1992, 68, 1145-1162.

169. Glanville, J.; Zhai, W.W.; Berka, J.; Telman, D.; Huerta, G.; Mehta, G.R.; Ni, I.; Mei, L.; Sundar, P.D.; Day, G.M.R.; Cox, D.; Rajpal, A.; Pons, J. Precise Determination of the Diversity of a Combinatorial Antibody Library Gives Insight into the Human Immunoglobulin Repertoire. Proc. Natl. Acad. Sci. U. S. A. 2009, 1, 20216-20221.

170. Tonegawa, S. Somatic Generation of Antibody Diversity. Nature 1983, 302, 575-581. 
171. Tonegawa, S. Susumu Tonegawa, Nobel Prize in Physiology or Medicine 1987. Available online: http://nobelprize.org/nobel_prizes/medicine/laureates/1987/tonegawa-autobio.html (accessed on March 20, 2010).

172. Press Release for Nobelförsamlingen Karolinska Institutet, the Nobel Assembly at the Karolinska Institute 1987. Available online: http://nobelprize.org/nobel_prizes/medicine/ laureates/1987/press.html (accessed on March 20, 2010).

173. Porch, B.; de Berkeley-Wykes, J. Bilingual aphasia and Its Implications for Cerebral Organization. In Clinical Aphasiology; Brookshire, R., Ed.; BRK Publishers: Minneapolis, MN, USA, 1985; Volume 15; pp. 107-112. Available online: http://aphasiology.pitt.edu/ archive/00000843/01/15-14.pdf (accessed on March 20, 2010).

(C) 2010 by the authors; licensee Molecular Diversity Preservation International, Basel, Switzerland. This article is an open-access article distributed under the terms and conditions of the Creative Commons Attribution license (http://creativecommons.org/licenses/by/3.0/). 